\title{
Spatial and temporal autocorrelation weave human brain networks
}

Maxwell Shinn ${ }^{1,2}$, Amber $\mathrm{Hu}^{3}$, Laurel Turner ${ }^{3}$, Stephanie Noble ${ }^{4}$, Sophie Achard ${ }^{5}$, Alan Anticevic ${ }^{1,2}$, Dustin Scheinost ${ }^{1,4}$, R. Todd Constable ${ }^{1,4}$, Daeyeol Lee Le,7,8,9 $^{\text {, }}$ Edward T. Bullmore ${ }^{10 *}$, John D. Murray ${ }^{1,2,11 *}$

\author{
1 Interdepartmental Neuroscience Program, Yale University \\ 2 Department of Psychiatry, Yale University \\ 3 Yale College, Yale University \\ 4 Department of Radiology and Biomedical Imaging, Yale University \\ 5 University of Grenoble Alpes, CNRS, Inria, Grenoble INP, LJK, 38000 Grenoble, France \\ 6 Zanvyl Krieger Mind/Brain Institute, Johns Hopkins University \\ 7 Kavli Discovery Neuroscience Institute, Johns Hopkins University \\ 8 Department of Psychological and Brain Sciences, Johns Hopkins University \\ 9 Department of Neuroscience, Johns Hopkins University \\ 10 Department of Psychiatry, University of Cambridge \\ 11 Department of Physics, Yale University
}

Correspondence: etb23@cam.ac.uk, john.murray@yale.edu

June 1, 2021

\begin{abstract}
Correlations are a basic object of analysis across neuroscience, but multivariate patterns of correlations can be difficult to interpret. For example, correlations are fundamental to understanding timeseries derived from resting-state functional magnetic resonance imaging (rs-fMRI), a proxy of brain activity. Networks constructed from regional correlations in rs-fMRI timeseries are often interpreted as brain connectivity, yet the links between brain networks and neurobiology have until now been largely speculative. Here, we show that the topology of rs-fMRI brain networks is caused by the spatial and temporal autocorrelation of the timeseries used to construct them. Spatial and temporal autocorrelation show high test-retest reliability, and are correlated with popular measures of network topology. A generative model of spatially and temporally autocorrelated timeseries exhibits similar network topology to brain networks, and when fit to individual subjects, it captures near the reliability limit of subject and regional variation. We demonstrate why spatial and temporal autocorrelation induce network structure, and highlight their ability to link graph properties to neurobiology during healthy aging. These results offer a reductionistic account of brain network complexity, explaining characteristic patterns in brain networks using timeseries statistics.
\end{abstract}

Patterns in high-dimensional data can be difficult to quantify. Network neuroscience approaches high dimensionality in brain data using a graph theory framework to analyze the brain's structural and functional connections [1-3]. In rsfMRI, timeseries can be measured for each brain region, and the pairwise correlations of these timeseries, the functional connectivity (FC) matrix (Figure 1a), is thought to distinctively characterize brain connectivity [4]. Thus, a graph constructed directly from the FC matrix (a weighted graph), or more commonly from the FC matrix binarized to contain only the strongest fixed fraction of values (unweighted graph), encapsulates patterns of brainwide connectivity (Figure 1a). In these graphs, nodes represent brain regions, and edges linking the nodes represent the correlation between the regions' rs-fMRI timeseries. Graph metrics are statistics quantifying the graph's topology [1,5] (Figure 1a). In principle, graph abstractions allow brain connectivity to be studied with the same framework no matter how the graph was constructed, although in practice, graph topology depends heavily on the methodology used to define connectivity $[3,6,7]$. Uncovering patterns in graph structure may reveal rules governing brainwide functional organization.

\section{Hidden structure in brain networks}

We found that a small set of neurobiologically interpretable factors underlie individual variation in rs-fMRI graph topol- ogy. We analyzed multiple neuroimaging datasets utilizing diverse methodologies implemented by different teams, focusing on the Human Connectome Project (HCP) dataset [8] with validation in the Yale Test-Retest (TRT) [9] and Cambridge Centre for Ageing and Neuroscience (Cam-CAN) [10,11] datasets. In all datasets, graph metrics are highly correlated with each other across subjects (Figure 1b, S1a,i,q) [12-15], implying a low-dimensional set of factors drives individual variation in graph metrics. However, unweighted graph metrics are also highly correlated with the mean (mean-FC), variance (var-FC), and kurtosis (kurt-FC) of the FC matrix (Figure $1 \mathrm{~b}, \mathrm{~S} 1 \mathrm{a}, \mathrm{i}, \mathrm{q})$. This is surprising because the binarization procedure ensures that all unweighted graphs have the same number of edges, and hence, graphs contain no explicit information about mean-FC, var-FC, or kurt-FC. Thus, some hidden underlying factors must influence both the distribution of FC values and the topology of the unweighted graph. In what follows, we demonstrate that spatial and temporal autocorrelation are two such factors.

\section{Spatial autocorrelation}

Spatial autocorrelation (SA) is the ubiquitous phenomenon in neuroscience that nearby regions are more correlated than distant regions [16-21]. In order to examine SA's test-retest reliability, i.e. how well SA is preserved across different rs-fMRI sessions from the same subject, we developed a method to es- 

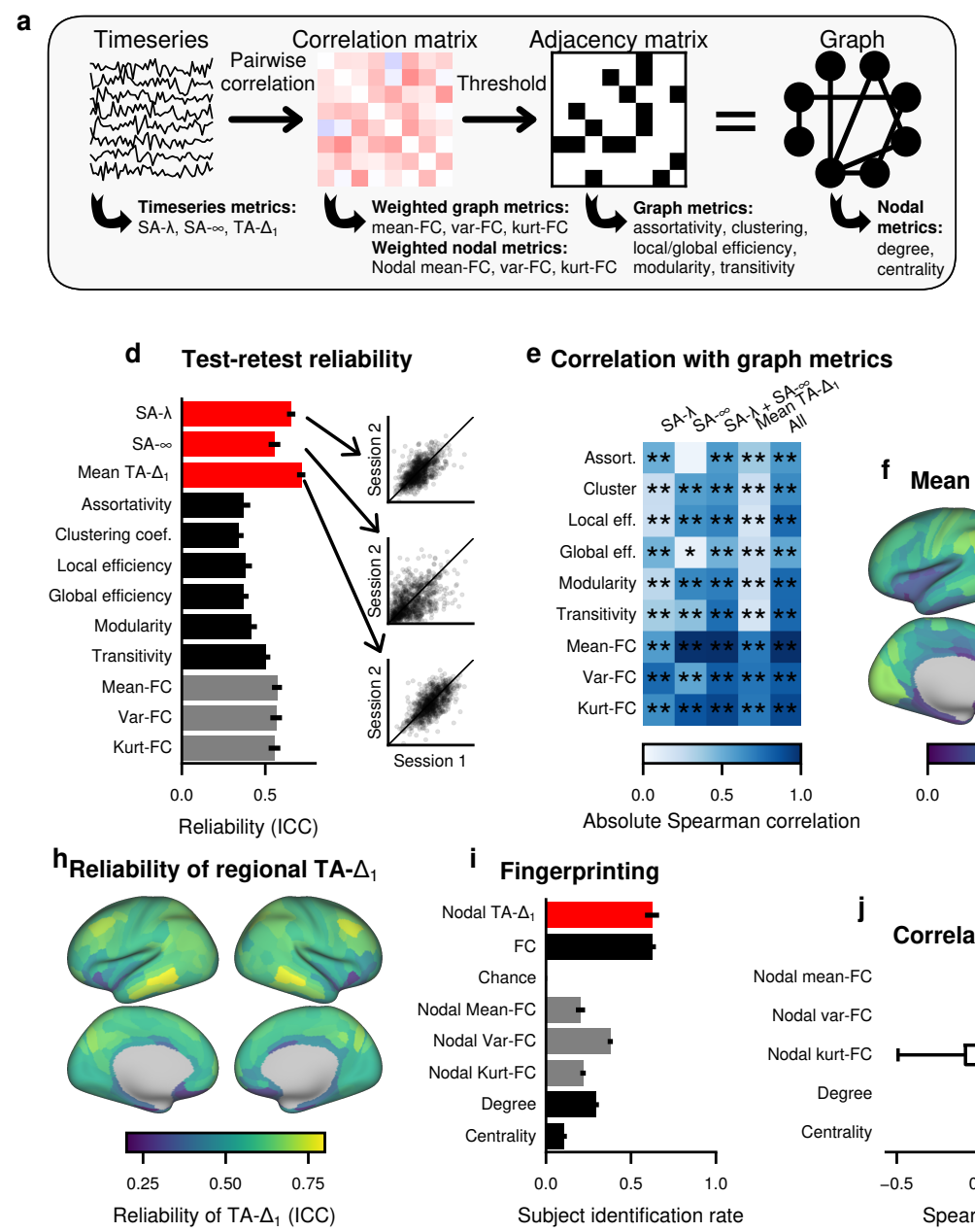

\section{e Correlation with graph metrics}

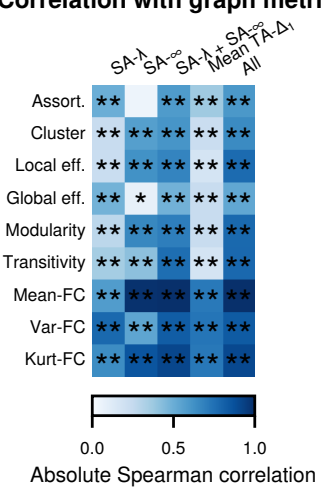

Absolute Spearman correlation
Correlation among graph metrics

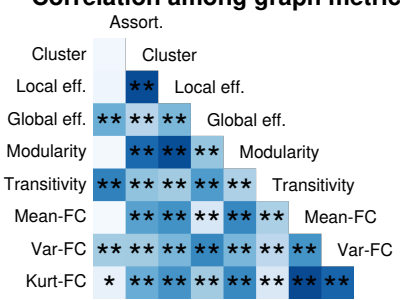

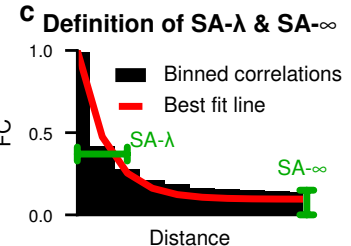

Distance

Definition of TA- $\Delta_{1}$

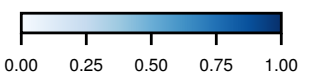
Absolute Spearman correlation

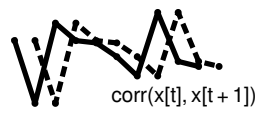

f Mean regional TA- $\Delta_{1}$
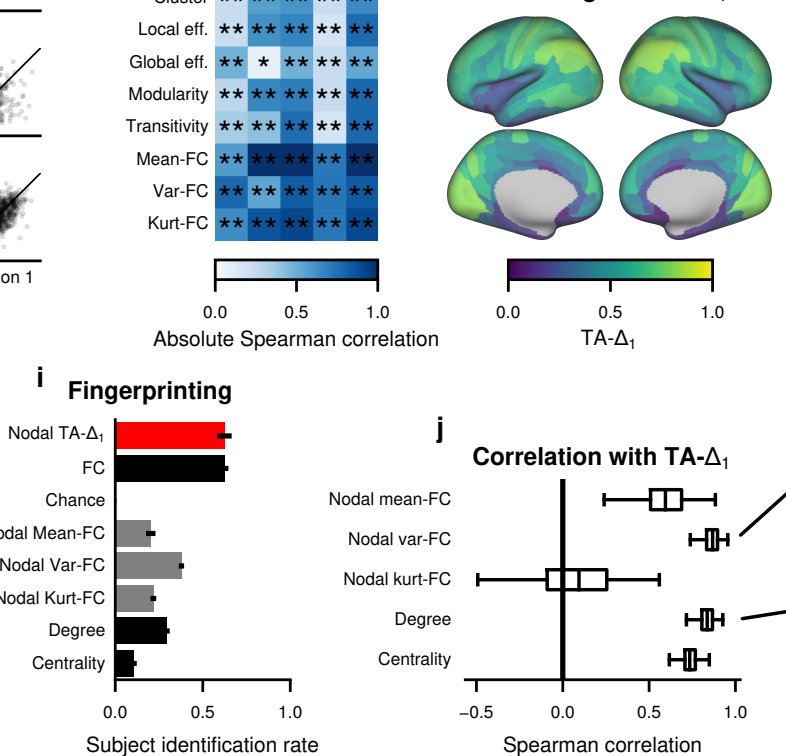

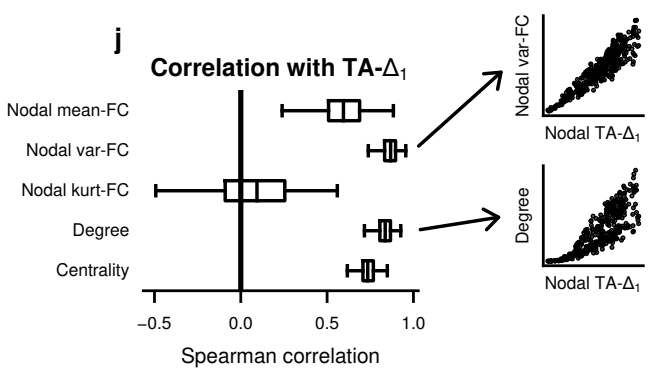

Reliability of nodal metrics

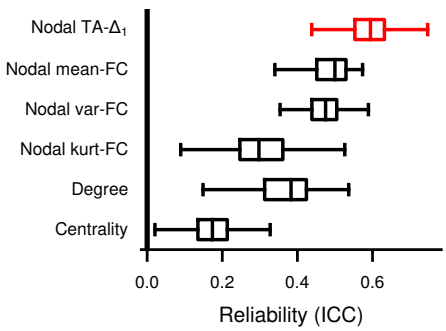

k

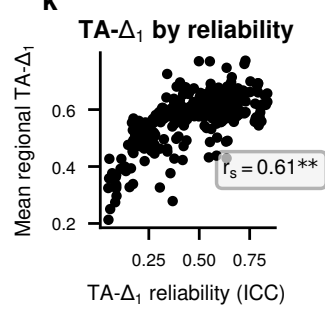

Figure 1 | Spatial and temporal autocorrelation are important features of rs-fMRI timeseries. (a) Diagram describing the connectomic pipeline and key graph metrics. (b) Correlation of graph metrics across all subjects in the HCP dataset. * indicates $p<0.05$ and ${ }^{* *}$ indicates $p<0.01$. (c) Schematic demonstrating the calculation of SA- $\lambda$ and $S A-\infty$ (top) and TA- $\Delta_{1}$ (bottom). (d) Test-retest reliability of graph metrics, quantified by intraclass correlation coefficient (ICC). Error bars indicate $95 \%$ confidence interval. Inset scatterplots show the correlation across subjects for two example sessions. (e) Correlation across subjects between weighted or unweighted graph metrics and SA- $\lambda, \mathrm{SA}-\infty$, or TA- $\Delta_{1}$. "SA- $\lambda$ + SA- $\infty$ " indicates a cross-validated linear model with these two terms, and "all" indicates a cross-validated linear model with all three terms. * indicates $p<0.05$ and ${ }^{* *}$ indicates $p<0.01$. (f) Mean TA- $\Delta_{1}$ for each brain region. (g) Reliability is computed for each nodal graph metric separately in each region. Boxplots indicate the distribution of ICC across regions. (h) Reliability of TA- $\Delta_{1}$ is plotted across brain regions. (i) A fingerprinting analysis to identify individual subjects is computed for each of six possible pairs of the four sessions. The mean fraction of subjects correctly identified is shown, with error bars indicating standard error. (j) The correlation across regions of regional TA- $\Delta_{1}$ with nodal weighted and unweighted graph metrics was computed for each subject, and boxplots indicate the distribution across subjects for each metric. (k) TA- $\Delta_{1}$ for each region, shown in (f), is plotted against the reliability of TA- $\Delta_{1}$ for each region, shown in (h).

timate SA on a single-subject level by decomposing it into two components: the rate at which FC falls off with physical distance (SA- $\lambda$ ), and the average correlation between two distant brain regions $(S A-\infty)$. Our method bins FC values by distance and finds the best fit SA- $\lambda$ and SA- $\infty$ for each subject's FC vs distance curve (Figure 1c), using Euclidean distance to accommodate both hemispheres and subcortex (Equation 1). Test-retest reliability is quantified with intraclass correlation coefficient (ICC), which compares the variability of multiple observations of the same subject to the variation across all subjects, where 1 is perfect reliability and 0 is chance.

We found that SA is both reliable and correlated with graph metrics across subjects. Both SA- $\lambda$ and SA- $\infty$ have high test-retest reliability, compared to graph metrics (Figure 1d, $\mathrm{S} 1 \mathrm{~b}, \mathrm{j})$. This cannot be explained by head motion within the scanner (accounting for motion, partial correlation $>.65, p<$
$10^{-10}$ for both SA- $\lambda$ and SA- $\infty$ ) or global signal regression (Figure S1), and is strongest in the HCP dataset, which had the smallest motion confounds (Figure S3a,b). Both SA- $\lambda$ and SA- $\infty$ are highly correlated with weighted and unweighted graph metrics (Figure 1e, S1c,k,r), and a linear model composed of $S A-\lambda$ and $S A-\infty$ significantly predicts all considered graph metrics on held-out data (Figure 1e, S1c,k,r). Therefore, SA is reliable and topologically informative.

\section{Temporal autocorrelation}

Temporal autocorrelation (TA) describes the smoothness, or memory, of the rs-fMRI timeseries over time, and is known to vary heterogeneously across brain regions [6,22-27]. We quantify TA for each parcellated region in each subject as the Pearson correlation between adjacent time points of the region's timeseries, i.e. the lag-1 temporal autocorrelation 
(TA- $\Delta_{1}$ ) (Figure 1c). TA- $\Delta_{1}$ is a simple non-parametric measure of TA, which is related to other TA measures such as parametric estimates of long-memory dynamics (Figure S2) (Supplement 1) $[27,28]$. We observed highest TA- $\Delta_{1}$ in occipital and parietal regions, and lowest in limbic regions (Figure $1 \mathrm{f}, \mathrm{S} 1 \mathrm{~d}, 1, \mathrm{~s})$.

TA is reliable at both the subject and regional levels. At the subject level, we computed each subject's mean TA- $\Delta_{1}$ by averaging across all regions, and found that mean TA- $\Delta_{1}$ is highly reliable compared to graph metrics (Figure 1d, S1b,j). At the regional level, we computed the ICC across subjects for each region separately, and found that median regional reliability was higher for TA- $\Delta_{1}$ than for other nodal graph metrics (Figure 1g, S1e,m). The regional reliability of TA- $\Delta_{1}$ varies heterogeneously across the brain (Figure 1h) [29].

The reliability and heterogeneity of TA- $\Delta_{1}$ hints that it could be used to identify individual subjects across the population. To identify subjects, we utilized a "fingerprinting" analysis [9,30]. For a given measure, such as TA- $\Delta_{1}$, we matched each session of each subject to the session with the highest Pearson correlation in that measure across regions, selecting among all sessions from all subjects. Then, we counted the number of pairs for which both sessions belonged to the same subject. We found that fingerprinting using regional TA- $\Delta_{1}$ identified the single matching session from the pool of 1765 sessions in over $62.4 \%$ of subjects, compared to $62.6 \%$ with a more traditional fingerprinting analysis using the entire FC matrix (Figure 1i), with similar success in other datasets (Figure S1f,n). We compared this to fingerprinting using several nodal graph metrics, which were unable to match the performance (Figure 1i, S1f,n). This indicates that TA- $\Delta_{1}$ is reliable enough to identify an individual subject from a population, and confirms the subject specificity of $\mathrm{TA}-\Delta_{1}$.

TA- $\Delta_{1}$ is highly correlated with graph topology at the subject and regional level. At the subject level, we found a strong correlation between mean TA- $\Delta_{1}$ and graph metrics (Figure 1e) and a linear model incorporating TA- $\Delta_{1}$ with SA- $\lambda$ and SA- $\infty$ significantly predicted all graph metrics. This effect was strongest in the HCP dataset, which had the smallest motion and parcel size confounds (Figure S3c,d). At the regional level, for each subject, we computed the Spearman correlation between regional TA- $\Delta_{1}$ and various graph metrics. We found that both weighted and unweighted nodal graph metrics were highly correlated with TA- $\Delta_{1}$ (Figure $1 \mathrm{j}, \mathrm{S} 1 \mathrm{~g}, \mathrm{o}, \mathrm{t}$ ). Most notably, a node's degree, or the total number of connections a node makes to other regions, was predicted by TA- $\Delta_{1}$ with a median correlation of 0.89 (Figure 1j), and this relationship was not driven by parcel size (median partial correlation 0.83 ). Remarkably, this implies that network hubs can be discovered without examining the topology of network.

\section{Multiple sources of temporal autocorrelation}

TA in fMRI can be shaped by multiple underlying factors, such as the noise level of a parcel. For any temporallyautocorrelated timeseries, adding uncorrelated noise to the timeseries reduces its TA. Therefore, if the entire brain had a slow underlying timescale-for example, due to the slow hemodynamic response-and some regions were noisier than others, then TA would be lower in regions with the most noise.
Thus, a region's TA partially reflects the amount of noise present [31]. Consistent with this hypothesis, we found that regions with the lowest TA- $\Delta_{1}$ show the lowest reliability in TA- $\Delta_{1}$ (Figure $1 \mathrm{k}, \mathrm{S} 1 \mathrm{~h}, \mathrm{p}$ ). By this logic, TA may influence FC by altering the fraction of shared variance between pairs of regions [32]. In what follows, we directly tested the hypothesis that noise drives TA, by building a generative model with regionally heterogeneous noise.

\section{Generative model of autocorrelated timeseries}

We demonstrated a causal relationship of SA and TA to network topology by fitting individual subjects with a parsimonious generative model of timeseries with SA and TA. The model operates at the level of the parcellated timeseries, meaning that comparisons may be drawn at all levels of the analysis pipeline (Figure 1a). Our model can be summarized in a small number of steps (Figure 2a). We generate longmemory $\left(1 / f^{\alpha}\right.$ spectrum) timeseries $[28,33,34]$ which have uniformly high TA. Concurrently, we spatially embed these timeseries according to brain geometry, introducing SA by increasing the correlation of nearby regions, while preserving the frequency spectra using a newly-derived algorithm (Supplement 2.1). Then, we add uncorrelated white noise with a region-specific variance, thereby lowering TA heterogeneously (Supplement 2.3). The resulting timeseries can then be analyzed the same way as rs-fMRI timeseries. Thus, in our model, all variation in graph topology must be caused by variation in SA and/or TA.

Our model contains two SA parameters which are fit on the single-subject level, corresponding to noiseless SA- $\lambda$ $\left(\mathrm{SA}-\lambda^{{ }^{\text {gen }}}\right)$ and noiseless SA- $\infty$ (SA- $\left.\infty^{\text {gen }}\right)$ (Supplement 2.1). We also utilize the subject's observed regional TA- $\Delta_{1}$ to determine the variance of noise to add in the final step (Supplement 2.3). Since it was not possible to estimate SA- $\lambda^{\text {gen }}$ and SA- $\infty^{\text {gen }}$ directly from the data (Supplement 2.4), we fit them through optimization to a feature of the data we do not directly assess, the eigenvalue distribution, which is well-captured by the model (Figure 2b). This parameterization ensures that all variation in graph topology must be caused by variation in SA and/or TA.

\section{Model performance}

We evaluated the ability of our model to capture weighted and unweighted graph metrics. In principle, model fit could be assessed using several different criteria, such as the model's ability to capture as much variance as possible (e.g. using $R^{2}$ ), or alternatively, by its ability to capture individual variation (e.g. using Pearson correlation) (Table S1). Here, we assess model fit using Lin's concordance, a stricter criterion which is 1 only when the model captures both variance and individual variation, and zero when either assessment is poor (Figure 2c). An example subject's network and an example model network for that subject is shown in Figure 2d.

Our model captures important features of graph topology. As expected, we confirmed the model matches the SA and TA of the original subjects (Figure S5). The model captures weighted and unweighted graph metrics, with similar Lin's concordance as a second session from the same subject (Figure 2e, S6). It also reproduces the degree distribution (Figure $2 \mathrm{f})$, i.e., the fraction of nodes with a given degree, and cap- 
a

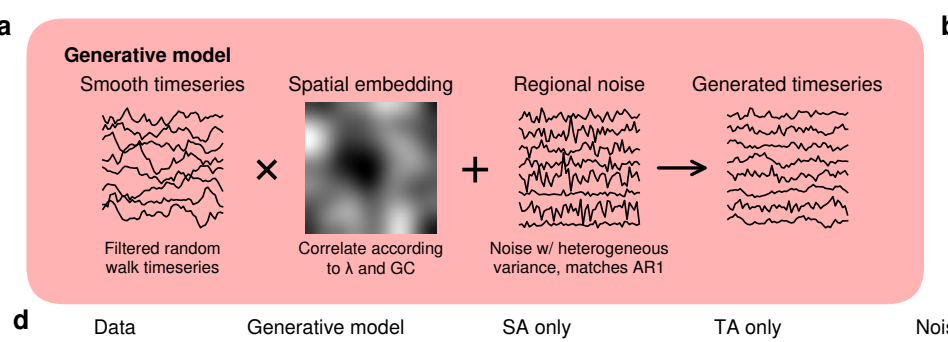

b

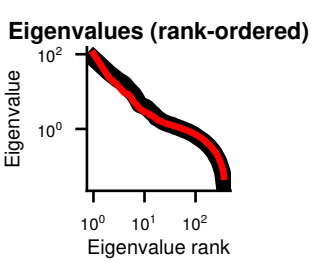

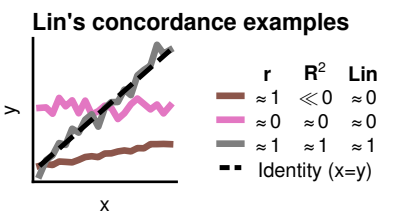
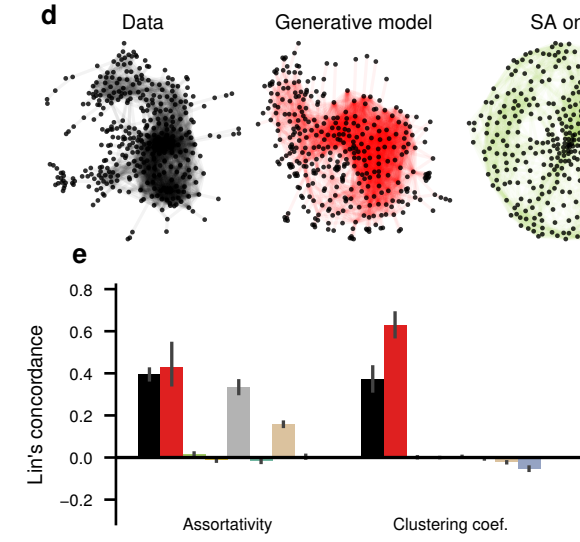

Assortativity

Clustering coef.

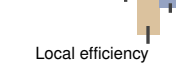

Global efficiency

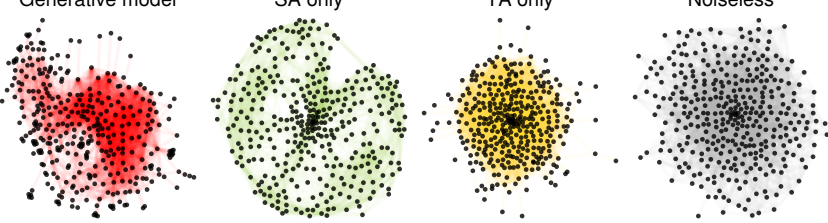

Model-data similarity

Phase randomization

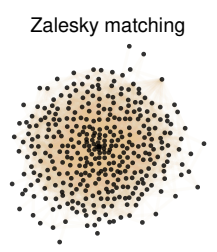

Edge reshuffle
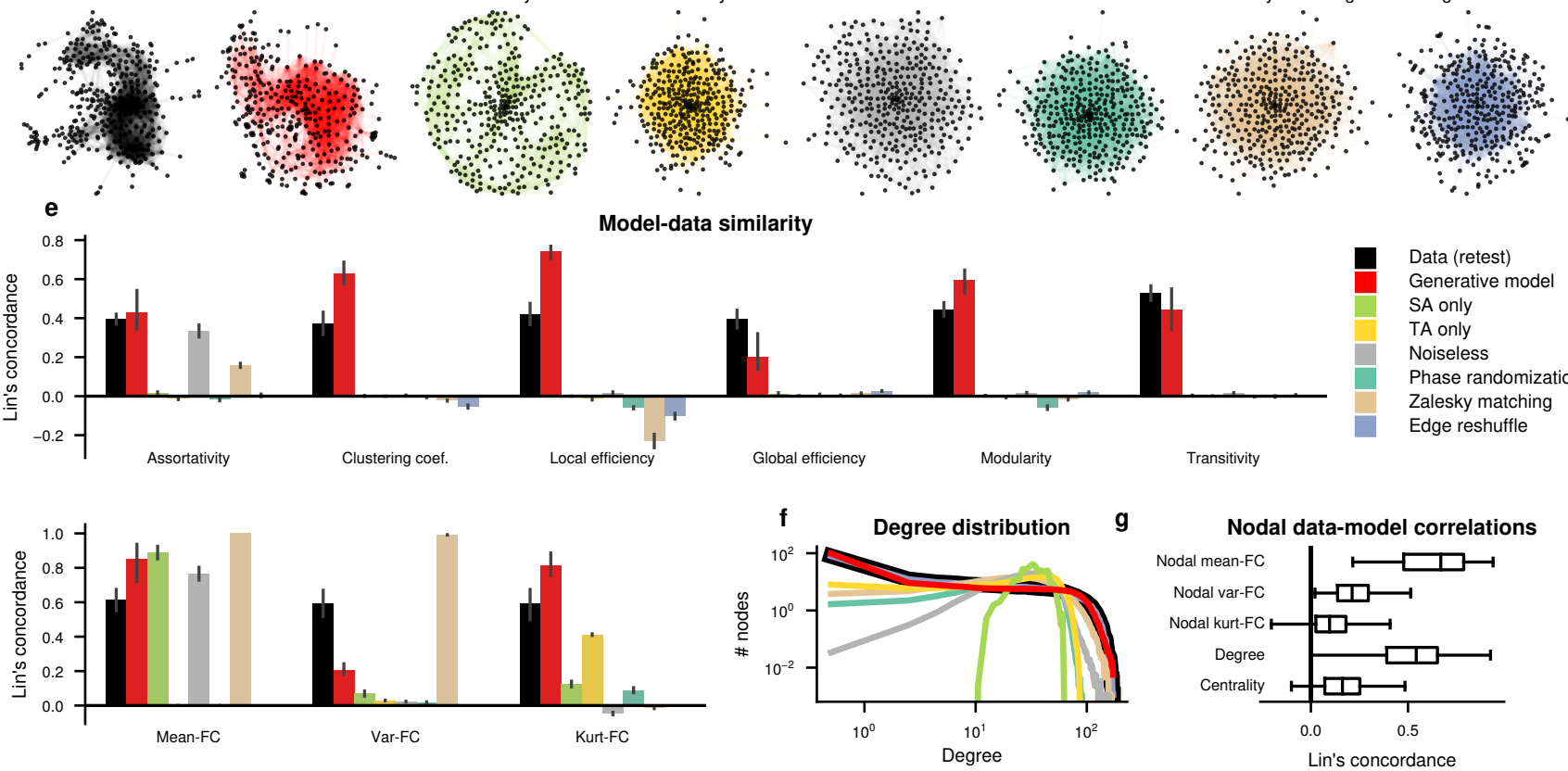

Transitivity

Data (retest)

Generative model

SA only

TA only

Noiseless

Phase randomization

Zalesky matching

Edge reshuffle

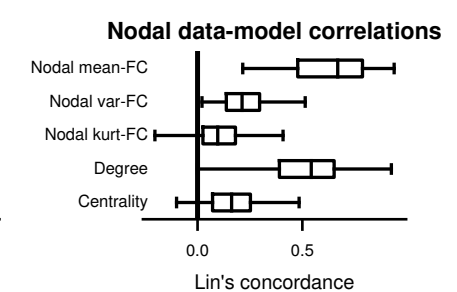

Figure 2 | A generative model captures connectome topology. (a) Schematic of our generative modeling framework for timeseries with SA and regionally heterogeneous TA. (b) Log-log distribution of FC matrix eigenvalues for an example subject (black) compared to the generative model (red). (c) A schematic demonstrating Lin's concordance, our model fit statistic. (d) Graphs for the original data (black) and for each model (colors) visualized using a force-directed layout, which positions topographically neighboring nodes nearby. (e) Lin's concordance between model and data for each model. Bars represent the mean across the four scanning sessions, and error bars the standard error. For comparison, black indicates Lin's concordance between sessions from the same subject. (f) Log-log degree distribution for each model compared to the data (black). (g) Distribution of Lin's concordance of nodal metrics between model and data for each region.

tures regional graph metrics (Figure 2g).

To confirm that a simpler model is unable to match this performance, we fit versions of the model which capture only SA ("SA only"), only TA ("TA only"), or both SA and TA but with TA determined by intrinsic timescale instead of noise ("Noiseless") (Figure S4). We also fit several commonly used null models: one which preserves the power spectrum amplitudes ("Phase randomization"), one which preserves mean-FC and var-FC ("Zalesky matching") [6], and one which preserves degree distribution ("Edge reshuffle") (Figure S4). None of these alternative models capture network topology as measured by weighted and unweighted graph metrics (Figure 2e, S5, S6, Table S1) and the degree distribution (Figure 2f). The ability of our generative model to generally fit the TRT and Cam-CAN datasets better than alternative models emphasizes its generality (Figure S7a,d,g, Table S1).

\section{Linking spatial and temporal autocorrelation to graph topology}

So far, we have demonstrated that SA and TA predict graph topology, but not why this is the case. The impact of SA and TA on graph topology can be understood intuitively by considering how they impact the formation of individual edges. The impact of SA is relatively straightforward-SA increases the mean correlation between nearby regions, and thus, increases the probability of an edge. However, it is not immediately clear how TA might influence topology.

One link between TA and graph topology can be explained through binarization. We previously showed that a node's degree is highly correlated with its TA- $\Delta_{1}$ (Figure $1 \mathrm{j}$ ). Degree is determined by the number of correlations the node makes which exceed the binarization threshold (Figure 1a). This means that even if the expected value of the correlation (nodal mean-FC) is low, there may still be several correlations above the threshold if it has a high variance (nodal var-FC). Two temporally autocorrelated timeseries are expected to have a higher variance in their pairwise correlation [35] (Supplement 3), as reflected in our data (Figure 1e,j). Thus, for individual nodes, one way that TA drives a node's degree is by increasing the var-FC. At the level of the graph, if region A is highly correlated with regions $\mathrm{B}$ and $\mathrm{C}$, it is more likely that $\mathrm{A}$ and $\mathrm{C}$ are correlated with each other [6]. These observations collectively creates a clustered topology, whereby high-TA- $\Delta_{1}$ regions are more likely to be connected to each other.

We confirmed this reasoning using a two-parameter "economical clustering" model [14] which builds graphs by proba- 
bilistically connecting nodes based on their distance and clustering topology (Supplement 4). This model reproduces several topological features of brain networks [13-15], and is convenient because the procedure for constructing networks is distinct from the rs-fMRI pipeline. We found that changes in SA and TA correspond to an increased propensity for short and clustered edges, respectively, in the economical clustering model (Supplement 4) (Figure S8). The impact of these parameters on graph metrics is also qualitatively similar in the two models (Figure S9). Thus, SA and TA can be interpreted in terms of graph topology.

\section{Spatial and temporal autocorrelation in healthy ag- ing}

SA and TA can link graph properties to neurobiology, as we demonstrate in healthy aging. We analyzed the Cam-CAN dataset, containing cross-sectional rs-fMRI data from over 800 subjects ranging from age 18 to 90 . Since motion was highly correlated with age in this dataset (Figure S3e), we performed all analyses using partial correlation controlling for motion. Several weighted and unweighted graph metrics are correlated with age (Figure S3), with global efficiency, varFC, and kurt-FC showing significant correlations. Age was positively correlated with SA- $\lambda$, negatively correlated with SA- $\infty$, and uncorrelated with mean TA- $\Delta_{1}$ (Figure $3 a$ ).

Our generative model reveals that the impact of aging on network topology can be captured by the influence of age on SA- $\infty$ alone. Since SA- $\infty$ decreases with age, we perturbed the model by decreasing SA- $\infty^{\text {gen }}$ to obtain model predictions for SA- $\infty$-mediated aging. We found that the predictions match all significant correlations between age and graph metrics from the data (Figure $3 b$ ). By contrast, since SA- $\lambda$ increases with age, we also perturbed the model by increasing SA- $\lambda^{\text {gen }}$ to obtain predictions for SA- $\lambda$-mediated aging. However, these predictions do not match the data (Figure $3 \mathrm{~b}$ ). This suggests that the impact of aging on network structure is mediated by changes in SA- $\infty$, i.e. by disproportionate strengthening of long-distance connections.

While mean TA- $\Delta_{1}$ did not change with age, different regions exhibited different patterns. We calculated the partial correlation of age and regional TA- $\Delta_{1}$ across the brain for each brain region (Figure 3c). Regions in the frontal subnetwork showed significant decreases in TA- $\Delta_{1}$ with age $(29 \%$ of regions significant, $\mathrm{p}<.05)$, while cerebellar regions showed increases with age $(44 \%$ of regions significant, $\mathrm{p}<.05)$ (Figure $3 d)$. This suggests a regional specificity in the effects of aging.

\section{Discussion}

Here, we have shown that spatial and temporal autocorrelation-as parameterized by $\mathrm{SA}-\lambda, \mathrm{SA}-\infty$, and TA- $\Delta_{1}$ - are highly reliable properties of rs-fMRI timeseries that correlate with, and are predictive of, connectome topology. A generative model incorporating SA and TA can reproduce individual variation in connectome topology, establishing a causal link between SA, TA, and network topology. This link can be understood intuitively by considering the impact of SA and TA on the network. Furthermore, the generative model pinpoints changes in graph metrics to the long-distance component of SA.

Our work raises an important question: if SA and TA
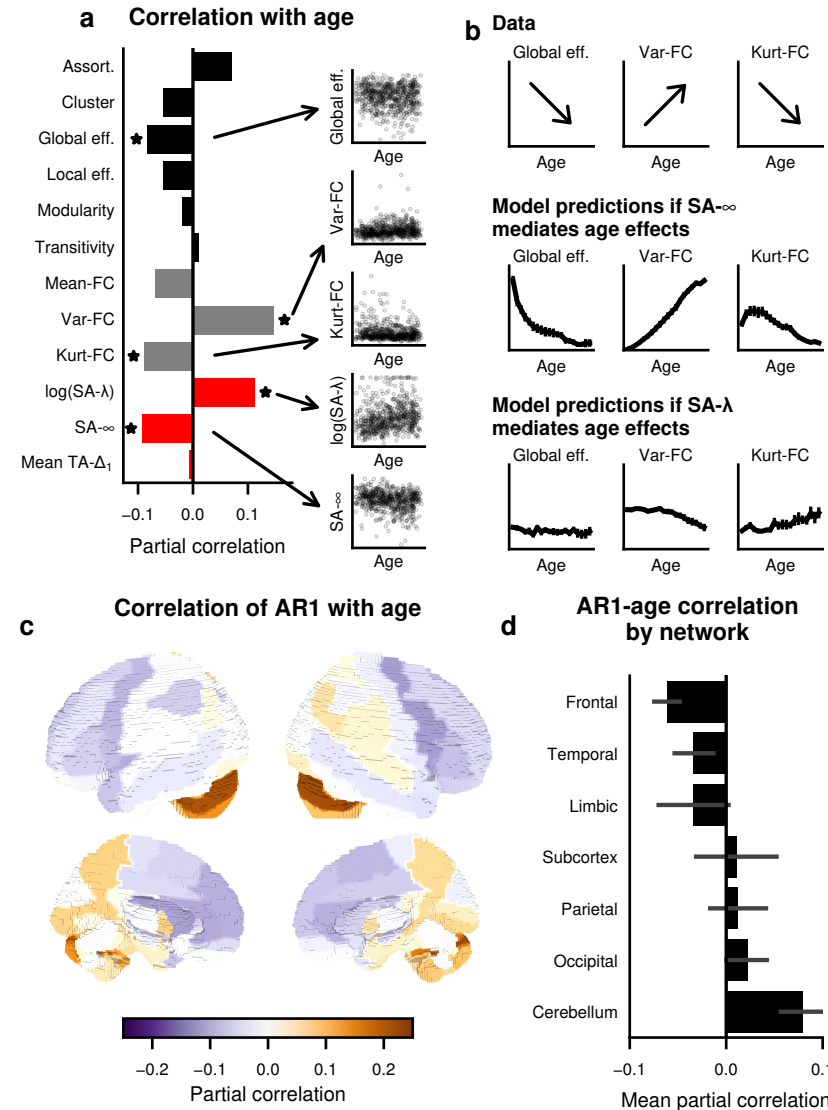

Model predictions if SA- $\lambda$ mediates age effects Giales age elfects

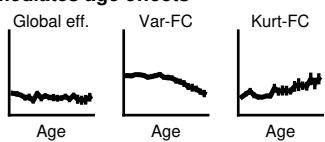

d AR1-age correlation by network

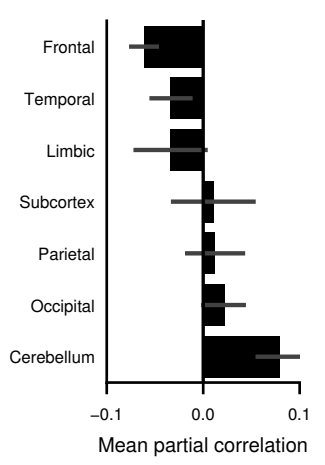

Figure 3 | Spatial and temporal autocorrelation link functional connectome topology to neurobiology in healthy aging. (a) Partial correlation of graph metrics with age is shown, controlling for motion. Asterisks indicate significance of partial correlation $(p<.05)$. (b) The generative model can be perturbed to test whether each SA parameter mediates aging. (top) Subject data predict global efficiency and kurt-FC decrease with age, whereas var-FC increases with age. The $S A-\infty$ and $S A-\lambda$ parameters were separately perturbed in the generative model according to how they change with age. The predicted change of each metric with age is shown if the age-related changes are due to $S A-\infty$ alone (middle) or SA- $\lambda$ alone (bottom). (c) Partial correlation of TA- $\Delta_{1}$ with age, controlling for motion, is shown for each region. (d) Partial correlations from (c) averaged across subnetworks. Error bars indicate $95 \%$ confidence interval.

drive graph metrics, then what drives SA and TA? There are numerous candidate mechanisms describing neurobiological sources for SA and TA, each of which can be investigated independently. For example, TA may be driven by the intrinsic timescale of a brain region, demonstrated at both the whole-brain and single-neuron level [23, 24, 26, 36, 37]. Alternatively, SA or TA may be linked to arousal [38-40]. Dynamic changes in SA and TA over time linked to biophysical signals such as pupil diameter or skin conductance may provide further insights without the need for animal models. Furthermore, it is also likely that anatomical structure serves as the scaffold for SA and TA [36,41-43], and thus, individual differences in brain morphology could explain our excellent test-retest reliability and fingerprinting performance. This could provide critical insights into the relationship between brain structure and function [20,27,44].

By contrast, methodological factors also influence SA and 
bioRxiv preprint doi: https://doi.org/10.1101/2021.06.01.446561; this version posted June 1, 2021. The copyright holder for this preprint (which was not certified by peer review) is the author/funder. All rights reserved. No reuse allowed without permission.

Shinn et al.

TA, and therefore, innovations in methodology are important for solidifying the link from network structure to biology. Prior studies have shown that methodological factors influence network topology $[6,7]$. We found that the influence of confounding factors - such as motion, parcel size, age, and regional variation in noise-depends on experimental design and preprocessing pipeline (Figure S3), consistent with prior findings $[21,45,46]$. A pipeline designed with SA and TA as variables of interest may mitigate these effects. Likewise, the influence of confounding physiological processes on SA and TA must be better understood $[47,48]$, including the link between TA and signal variability [49], especially related to aging [50]. Given the reliability and predictive power of SA and TA, solving these methodological issues makes SA and TA attractive as candidate biomarkers [36], especially for psychiatric and neurologic diseases for which biomarkers are scarce.

Our present study used a parcellated analysis, which limits the precision with which SA can be measured, and avoids explicitly modeling spatial inhomogeneities. SA- $\infty$ is conceptually similar to the global signal, but it remains reliable even after global signal regression, and thus may represent spatial inhomogeneities in the global signal. Our methods for computing both SA and TA scale well for voxel-level analyses, but fitting the generative model is computationally intractable for parcellations with many nodes. Additionally, our measure of SA and our generative model could both be adapted to alternative measures of distance, such as geodesic distance for a single hemisphere of cortex.

While we focus here on how SA and TA impact network topology using graph theory, the hidden structure induced by SA and TA must be present in resting-state functional connectivity in general. We use graph theory as a window to understanding this hidden structure. All analyses of restingstate functional connectivity may be influenced by SA and TA. Indeed, our findings echo those in rs-fMRI analyses more broadly [27,36]. Effects of SA and TA on rs-fMRI analysis can be mitigated through spatially- and temporally-informed null models [6, 20] (e.g. Figure 2a), reporting the confounds of experimental methodology and preprocessing on SA and TA (e.g. Figure S3), and adjusting for these factors in analyses, such as through partial correlation (e.g. Figure 3). Overall, we have shown that SA and TA offer a reductionistic account of functional connectivity. They present a rare link from graph topology to neurobiology, with important implications for reproducibility [21].

\section{Acknowledgments}

We thank Josh Burt for help with plotting; Amber Howell for assistance with HCP data; and Jean C.C. Vila for helpful discussions. Funding was provided by the Winston Churchill Foundation of the United States and the Gruber Foundation (MS); NIMH grant K00MH122372 (SN); grants from Agence Nationale de la Recherche (ANR-20-NEUC-003-01) and MIAI@Grenoble Alpes (ANR-19-P3IA-0003) (SA); an NIHR Senior Investigator award and the NIHR Cambridge Biomedical Research Centre (ETB); a SFARI Pilot Award (AA and JDM); and NIH grant R01MH112746 (JDM). Data collection and sharing for this project was provided in part by the Cambridge Centre for Ageing and Neuroscience (CamCAN). CamCAN funding was provided by the UK Biotech- nology and Biological Sciences Research Council (grant number BB/H008217/1), together with support from the UK Medical Research Council and University of Cambridge, UK.

\section{Author contributions}

MS and ETB conceived the research. MS designed the experiments. MS and AH performed the experiments. MS and JDM analyzed and interpreted results. SN, AA, DS, and RTC contributed methodology and resources. MS, LT, and SA performed the mathematical analysis. DL, ETB, and JDM provided supervision and funding. MS, AH, and LT wrote the first draft of the manuscript. All authors edited, revised, and approved the manuscript.

\section{Ethics declarations}

Competing interests DL is a co-founder of Neurogazer Inc. ETB serves on the scientific advisory board of Sosei Heptares and as a consultant for GlaxoSmithKline. AA and JDM serve on the technical advisory board of RBNC Therapeutics.

\section{References}

[1] Albert, R. \& Barabási, A.-L. Statistical mechanics of complex networks. Reviews of Modern Physics 74, 47-97 (2002).

[2] Bullmore, E. \& Sporns, O. Complex brain networks: graph theoretical analysis of structural and functional systems. Nature Reviews Neuroscience 10, 186-198 (2009).

[3] Fornito, A., Zalesky, A. \& Bullmore, E. Fundamentals of Brain Network Analysis (Academic Press, 2016).

[4] Buckner, R. L., Krienen, F. M. \& Yeo, B. T. T. Opportunities and limitations of intrinsic functional connectivity MRI. Nature Neuroscience 16, 832-837 (2013).

[5] Rubinov, M. \& Sporns, O. Complex network measures of brain connectivity: Uses and interpretations. NeuroImage 52, 1059-1069 (2010).

[6] Zalesky, A., Fornito, A. \& Bullmore, E. On the use of correlation as a measure of network connectivity. NeuroImage 60, 2096-2106 (2012).

[7] Cantwell, G. T. et al. Thresholding normally distributed data creates complex networks. Physical Review E 101 (2020).

[8] Glasser, M. F. et al. A multi-modal parcellation of human cerebral cortex. Nature 536, 171-178 (2016).

[9] Noble, S. et al. Influences on the test-retest reliability of functional connectivity MRI and its relationship with behavioral utility. Cerebral Cortex 27, 5415-5429 (2017).

[10] Shafto, M. A. et al. The cambridge centre for ageing and neuroscience (cam-CAN) study protocol: a cross-sectional, lifespan, multidisciplinary examination of healthy cognitive ageing. BMC Neurology 14 (2014).

[11] Taylor, J. R. et al. The cambridge centre for ageing and neuroscience (cam-CAN) data repository: Structural and functional MRI, MEG, and cognitive data from a cross-sectional adult lifespan sample. NeuroImage 144, 262-269 (2017)

[12] Li, C., Wang, H., de Haan, W., Stam, C. J. \& Mieghem, P. V. The correlation of metrics in complex networks with applications in functional brain networks. Journal of Statistical Mechanics: Theory and Experiment 2011, P11018 (2011).

[13] Morgan, S. E., Achard, S., Termenon, M., Bullmore, E. T. \& Vértes, P. E. Low-dimensional morphospace of topological motifs in human fMRI brain networks. Network Neuroscience 2, 285-302 (2018).

[14] Vértes, P. E. et al. Simple models of human brain functional networks. Proceedings of the National Academy of Sciences 109, 58685873 (2012).

[15] Betzel, R. F. et al. Generative models of the human connectome. Neurolmage 124, 1054-1064 (2016).

[16] Song, H. F., Kennedy, H. \& Wang, X.-J. Spatial embedding of structural similarity in the cerebral cortex. Proceedings of the National Academy of Sciences 111, 16580-16585 (2014).

[17] Wiedermann, M., Donges, J. F., Kurths, J. \& Donner, R. V. Spatial network surrogates for disentangling complex system structure from spatial embedding of nodes. Physical Review E 93, 042308 (2016).

[18] Roberts, J. A. et al. The contribution of geometry to the human connectome. NeuroImage 124, 379-393 (2016). 
[19] Eklund, A., Nichols, T. E. \& Knutsson, H. Cluster failure: Why fMRI inferences for spatial extent have inflated false-positive rates. Proceedings of the National Academy of Sciences 113, 7900-7905 (2016).

[20] Burt, J. B., Helmer, M., Shinn, M., Anticevic, A. \& Murray, J. D. Generative modeling of brain maps with spatial autocorrelation. NeuroImage 220, 117038 (2020).

[21] Botvinik-Nezer, R. et al. Variability in the analysis of a single neuroimaging dataset by many teams. Nature (2020).

[22] Fascianelli, V., Tsujimoto, S., Marcos, E. \& Genovesio, A. Autocorrelation structure in the macaque dorsolateral, but not orbital or polar, prefrontal cortex predicts response-coding strength in a visually cued strategy task. Cerebral Cortex 29, 230-241 (2017).

[23] Kiebel, S. J., Daunizeau, J. \& Friston, K. J. A hierarchy of time-scales and the brain. PLoS Computational Biology 4, e1000209 (2008).

[24] Murray, J. D. et al. A hierarchy of intrinsic timescales across primate cortex. Nature Neuroscience 17, 1661-1663 (2014).

[25] Arbabshirani, M. R. et al. Autoconnectivity: A new perspective on human brain function. Journal of Neuroscience Methods 323, 68-76 (2019).

[26] Spitmaan, M., Seo, H., Lee, D. \& Soltani, A. Multiple timescales of neural dynamics and integration of task-relevant signals across cortex. Proceedings of the National Academy of Sciences 117, 22522-22531 (2020).

[27] Shafiei, G. et al. Topographic gradients of intrinsic dynamics across neocortex. eLife 9 (2020).

[28] Maxim, V. et al. Fractional Gaussian noise, functional MRI and Alzheimer's disease. Neurolmage 25, 141-158 (2005).

[29] Keitel, A. \& Gross, J. Individual human brain areas can be identified from their characteristic spectral activation fingerprints. PLOS Biology 14, e1002498 (2016).

[30] Finn, E. S. et al. Functional connectome fingerprinting: identifying individuals using patterns of brain connectivity. Nature Neuroscience 18, 1664-1671 (2015).

[31] Noble, S., Scheinost, D. \& Constable, R. T. A decade of test-retest reliability of functional connectivity: A systematic review and metaanalysis. NeuroImage 203, 116157 (2019).

[32] Cole, M. W., Yang, G. J., Murray, J. D., Repov̌̌, G. \& Anticevic, A. Functional connectivity change as shared signal dynamics. Journal of Neuroscience Methods 259, 22-39 (2016).

[33] Bullmore, E. et al. Colored noise and computational inference in neurophysiological (fMRI) time series analysis: Resampling methods in time and wavelet domains. Human Brain Mapping 12, 61-78 (2001).

[34] Wagenmakers, E.-J., Farrell, S. \& Ratcliff, R. Estimation and interpretation of $1 / \mathrm{f} \alpha$ noise in human cognition. Psychonomic bulletin $\&$ review 11, 579-615 (2004).

[35] Afyouni, S., Smith, S. M. \& Nichols, T. E. Effective degrees of freedom of the Pearson's correlation coefficient under autocorrelation. NeuroImage 199, 609-625 (2019).

[36] Watanabe, T., Rees, G. \& Masuda, N. Atypical intrinsic neural timescale in autism. eLife $\mathbf{8}$ (2019).

[37] Raut, R. V., Snyder, A. Z. \& Raichle, M. E. Hierarchical dynamics as a macroscopic organizing principle of the human brain. Proceedings of the National Academy of Sciences 202003383 (2020).

[38] Huang, Z., Liu, X., Mashour, G. A. \& Hudetz, A. G. Timescales of intrinsic BOLD signal dynamics and functional connectivity in pharmacologic and neuropathologic states of unconsciousness. The Journal of Neuroscience 38, 2304-2317 (2018).

[39] Shafiei, G. et al. Dopamine signaling modulates the stability and integration of intrinsic brain networks. Cerebral Cortex 29, 397-409 (2018).

[40] Zerbi, V. et al. Rapid Reconfiguration of the Functional Connectome after Chemogenetic Locus Coeruleus Activation. Neuron 103, 702718.e5 (2019).

[41] Baria, A. et al. Linking human brain local activity fluctuations to structural and functional network architectures. NeuroImage 73, 144-155 (2013).

[42] Sethi, S. S., Zerbi, V., Wenderoth, N., Fornito, A. \& Fulcher, B. D. Structural connectome topology relates to regional BOLD signal dynamics in the mouse brain. Chaos: An Interdisciplinary Journal of Nonlinear Science 27, 047405 (2017).

[43] Fallon, J. et al. Timescales of spontaneous fMRI fluctuations relate to structural connectivity in the brain. Network Neuroscience 4, 788-806 (2020).
[44] Honey, C. J. et al. Predicting human resting-state functional connectivity from structural connectivity. Proceedings of the National Academy of Sciences 106, 2035-2040 (2009).

[45] Friston, K. J. et al. Analysis of fMRI Time-Series Revisited. NeuroImage 2, 45-53 (1995).

[46] Davey, C. E., Grayden, D. B., Egan, G. F. \& Johnston, L. A. Filtering induces correlation in fMRI resting state data. NeuroImage $\mathbf{6 4}, 728-740$ (2013).

[47] Chen, J. E. et al. Resting-state "physiological networks". NeuroImage 213, 116707 (2020).

[48] Drew, P. J., Mateo, C., Turner, K. L., Yu, X. \& Kleinfeld, D. Ultraslow oscillations in fMRI and resting-state connectivity: Neuronal and vascular contributions and technical confounds. Neuron 107, 782-804 (2020).

[49] Baracchini, G. et al. Inter-regional BOLD signal variability is an organizational feature of functional brain networks. NeuroImage 118149 (2021).

[50] Grady, C. L. \& Garrett, D. D. Understanding variability in the BOLD signal and why it matters for aging. Brain Imaging and Behavior $\mathbf{8}$, 274-283 (2013). 


\section{Online Methods}

Datasets We analyzed the following datasets, which comprise a diversity of preprocessing steps and experimental methodologies, including different parcellations, sampling rates, spatial and temporal smoothings, covariate regressions, and noise removal strategies.

Human Connectome Project (HCP) A total of 883 subjects aged 22-37 from the Human Connectome Project 1200 subject data release underwent four resting-state scanning sessions spread across two days. Resting-state scans lasted for 14.4 minutes with a TR of $0.72 \mathrm{~s}$ (sampling rate of $1.39 \mathrm{hz}$ ). Data were preprocessed with the Human Connectome Project minimal preprocessing pipeline. This includes distortion correction using the field map, realignment for head motion, registration to T1 images, and denoising using ICA-FIX, a technique based on independent component analysis to remove structured noise. This pipeline also included a high-pass filter at $0.01 \mathrm{hz}$. Data were parcellated into 360 regions (180 per hemisphere) [8] with multimodal surface matching based on MSMAll [52], and with $2 \mathrm{~mm}$ FWHM surface spatial smoothing constrained to the parcel. The first 100 timepoints were removed to ensure steady state.

Human Connectome Project with Global Signal Regression (HCP-GSR) Subjects and scan parameters are identical to the HCP dataset, but global signal regression was added as a preprocessing step before parcellation. Additionally, we did not truncate the first 100 TRs, for a total of 1200 timepoints. 33 additional subjects were excluded due to nonconvergence in the global signal regression pipeline for a total of 850 subjects.

Yale Test-Retest A total of 12 subjects were scanned on four different days, and six 6-minute sessions were performed each day. Each subject was scanned on two scanners, two days on each scanner, on days spaced approximately one week apart. Thus, half of the scanning sessions for each subject were performed on a different scanner. Scanning sessions lasted 6 minutes each with a TR of 1.0 (sampling rate $1.0 \mathrm{hz}$ ). Motion correction was applied, and images were spatially smoothed to achieve uniform spatial smoothness of a $2.5 \mathrm{~mm}$ Gaussian kernel $[53,54]$. Images were coregistered to a common subjectspecific space across days, and subsequently into MNI space, and parcellated using the Shen parcellation [55].

High pass filtering was performed by regressing out linear, quadratic, and cubic trends, and low pass filtering was performed with a Gaussian kernel with cutoff frequency of 0.19 hz. Mean white matter, mean cerebrospinal fluid, mean global signal, and a 24-parameter motion model were also regressed out of the data. No subjects, sessions, or regions were excluded.

Cambridge Centre for Ageing and Neuroscience (Cam-CAN) A total of 652 subjects were scanned using a TR of $1.97 \mathrm{~s}$ (sampling rate $0.508 \mathrm{hz}$ ). We utilized the standard preprocessing pipeline and parcellation provided by the Cam-CAN project $[10,11]$. In summary, scans underwent motion correction and slice time correction before coregistration to T1 images and normalization to MNI space with DARTEL. We utilized the default AAL parcellation provided by the CamCAN project Ref. [58]. We also applied a second-order But- terworth low-pass filter at half the Nyquist frequency $(0.127$ $\mathrm{Hz}$ ) to account for high-frequency motion artifacts. We excluded six subjects and one cerebellar region due to missing data, for a grand total of 646 subjects with 115 regions in the parcellation.

Estimating spatial autocorrelation We decomposed spatial autocorrelation into two components: the rate at which correlations decrease exponentially with distance (SA- $\lambda$ ), and the spatially-invariant level of correlation to which it decays $(\mathrm{SA}-\infty)$. This can be described quantitatively as

$$
\operatorname{corr}\left(x_{i}, x_{j}\right)=\mathrm{SA}-\infty+(1-\mathrm{SA}-\infty) e^{-d_{i, j} / \mathrm{SA}-\lambda}
$$

where $x_{i}$ is the timeseries for region $i, d_{i, j}$ is the Euclidean distance between regions $i$ and $j$, and corr is the Pearson correlation. The quantities SA- $\lambda$ and SA- $\infty$ are constants and do not depend on $i$ or $j$.

We estimated the parameters $S A-\lambda$ and $S A-\infty$ for each subject as follows. For each pair of brain regions, we computed both their physical Euclidean distance (from the region's centroid) as well as their Pearson correlation coefficient. We used Euclidean distance rather than geodesic distance because it is defined across cortical hemispheres and within subcortex. We binned each pair of brain regions according to their Euclidean distance, using $1 \mathrm{~mm}$ bins for the HCP and HCP-GSR datasets, and $5 \mathrm{~mm}$ bins for the TRT and Cam-CAN dataset due to the fewer number of parcels, and computed the mean Pearson correlation of pairs in each bin to generate correlation vs distance curve (Figure 1a). We then found the least square fit of Equation 1 to this curve, optimizing with gradient descent, bounding SA- $\lambda$ between 0 and 100 and SA- $\infty$ between -1 and 1 . While it is possible to fit Equation 1 directly to the distance and correlation of each pair without binning, our approach puts more weight on nearby and distant correlations, which are less represented in the data but most critical for determining SA- $\lambda$ and SA- $\infty$.

To account for heteroskedasticity in SA- $\lambda$ for the Cam-CAN dataset, we analyzed the logarithm of this parameter for this dataset.

Estimating temporal autocorrelation Our primary measure of temporal autocorrelation, TA- $\Delta_{1}$, is a non-parametric measurement computed by taking the Pearson correlation of neighboring timepoints in the parcellated timeseries, i.e., for a timeseries $x[t]$, we have $\operatorname{corr}(x[t], x[t+1])$. This measure is computationally efficient, and can be implemented with only a few lines of code. However, TA- $\Delta_{1}$ is not comparable across datasets due to differing TR.

FC matrix and graph construction We defined the FC matrix $\rho_{i, j}$ as the matrix of Pearson correlation coefficients $\rho_{i, j}=\operatorname{corr}\left(x_{i}, x_{j}\right)$ between each pairwise combination of regional timeseries $x_{i}$ and $x_{j}$.

We constructed unweighted, undirected graphs from model or data FC matrices using standard techniques [2,3]. We first constructed a spanning tree backbone to ensure connectedness by transforming each element of the FC matrix 
$\rho_{i, j}$ by $\sqrt{2\left(1-\rho_{i, j}\right)}$ - an operation which turns the correlation similarity measure into a distance [61] —and then applying Kruskal's algorithm to find the minimum spanning tree [62]. We iteratively added the strongest edges in the FC matrix to the spanning tree until the graph contained $10 \%$ of all possible edges (proportional thresholding). This produced an unweighted, undirected graph with a fixed number of edges.

Quantifying reliability We quantify univariate reliability using the intraclass correlation coefficient (ICC). ICC measures the reliability of a particular scalar measure across subjects $[31,63]$. Let $N$ be the total number of subjects, $M$ be the number of sessions per subject, and $\gamma_{n}^{m}$ be some scalar measure of interest for session $m$ of subject $n$. Similar to an ANOVA, ICC decomposes the variance across subjects into variance from a common source, $\sigma_{\gamma}^{2}$, and noise, $\sigma_{\epsilon}$, by assuming that $\gamma$ can be decomposed into a subject-specific term $\gamma_{n}$ and a noise term $\epsilon_{n, m}$, i.e., $\gamma_{n}^{m}=\gamma_{n}+\epsilon_{n, m}$. Then, the ICC is defined as the fraction of variance explained by the subjectspecific term, i.e.,

$$
\mathrm{ICC}=\frac{\sigma_{\gamma}}{\sigma_{\gamma}+\sigma_{\epsilon}}
$$

In theory, there are generalizations of the ICC which can accommodate additional sources of variance. Here, we use the simplest form, ICC $(1,1)$ [31].

We quantify multivariate reliability using fingerprinting, similar to that performed in Ref. $[9,30,67]$. Let $\gamma_{n}^{m}$ be a vector describing some measure (e.g. TA- $\Delta_{1}$ ) from a single session $m \in\{1 \ldots M\}$ for subject $n \in\{1 \ldots N\}$. For each session $m$ of each subject $n$, we compute

$$
\Gamma(n, m)=\underset{n^{\prime}}{\operatorname{argmax}}\left(\max _{m^{\prime},(n, m) \neq\left(n^{\prime}, m^{\prime}\right)} \operatorname{corr}\left(\gamma_{n}^{m}, \gamma_{n^{\prime}}^{m^{\prime}}\right)\right),
$$

where corr is Pearson correlation. The fingerprinting performance is given by

$$
\frac{1}{N M} \sum_{m, n} I_{\Gamma(n, m)=n}
$$

where $I$ is the indicator function. Under this measure, chance performance is $(M-1) /(N M-1)$. For regional measures, the length of vector $\gamma_{n}^{m}$ was equal to the number of regions in the parcellation $R$. For FC, the length of $\gamma_{n}^{m}$ was $R(R-1) / 2$, i.e. the number of distinct elements in the FC matrix.

In the HCP and HCP-GSR datasets, subjects were scanned four times over two days. For computing ICC, we used $M=$ 4. For fingerprinting, in order to obtain error estimates and increase the difficulty of fingerprinting, we performed fingerprinting on each possible pair of sessions 1-4 $(M=2$, performed six times, instead of $M=4$ performed once). This means each session had only one correct match of the 1765 sessions in the pool. In the TRT dataset, subjects were scanned in six sessions across four different days for a total of 24 sessions per subject. Likewise, we used $M=24$ to compute ICC. By contrast, we performed fingerprinting for each session independently (i.e. $M=4$ performed six times instead of $M=24$ performed once), so subjects had 3 correct matching sessions out of 47 in the pool. Without such increases in difficulty on these datasets, fingerprinting performance was near $100 \%$. We could not compute ICC or perform fingerprinting on the Cam-CAN dataset, since only one rs-fMRI session was acquired per subject.

Weighted graph metrics We consider three weighted graph metrics: the mean (mean-FC), variance (var-FC), and kurtosis (kurt-FC) of the FC matrix. Each is calculated by finding the corresponding statistic (mean, variance, or kurtosis) of the upper triangular portion of the FC matrix, excluding the diagonal.

Because TA can be measured for individual nodes, we also consider how it impacts local topology. To do this, we also define the corresponding nodal graph metrics for each which operates on rows of the FC matrix instead of the upper diagonal: nodal mean-FC, the mean of the row; nodal var-FC, the variance of the row; and nodal kurt-FC, the kurtosis of the row. We exclude self-connectivity (i.e. the diagonal of ones in the FC matrix) from these calculations.

Unweighted graph metrics We consider six popular graph metrics to quantify the topology of the connectome [5]:

Assortativity Assortativity is a preference of high-degree nodes to connect to each other. Mathematically, this is the Pearson correlation between the degree of nodes connected by edges [69].

Clustering coefficient The clustering coefficient for a single node is the average relative number of triangles around a node. For adjacency matrix $A$, the nodal clustering coefficient for node $i$ is

$$
\frac{1}{\left(\sum_{j} A_{i, j}\right)\left(\left(\sum_{j} A_{i, j}\right)-1\right)} \sum_{j, k, j \neq k} A_{i, j} A_{j, k} A_{i, k} .
$$

The clustering coefficient for the network is the average of the nodal clustering coefficients.

Global efficiency The global efficiency is related to the average topological distance between nodes. Mathematically, it is the mean of the inverse of shortest path lengths between each pair of nodes, i.e.

$$
\frac{1}{N(N-1)} \sum_{i, j, i \neq j} \frac{1}{s_{i, j}}
$$

where $s_{i, j}$ indicates the shortest path between nodes $i$ and $j$ and $N$ is the number of nodes [70].

Local efficiency Local efficiency, similar to clustering, is the mean global efficiency on the subgraph of each node's nearest neighbors [70].

Modularity Modularity quantifies the ability to break a network into "communities" such that the number of edges within the community is maximized and outside the community is minimized. Let $C_{i}$ be some community assignment of node $i$, and $\delta$ be the Kronecker delta function. We can compute the quality of the community assignment $C_{i}$ with the equation

$$
\sum_{i, j, i \neq j}\left(2 A_{i, j}-1\right)\left(2 \delta\left(C_{i}, C_{j}\right)-1\right) .
$$


The first term in the sum is 1 if the neurons are connected and -1 if they are not, and the second term is 1 if they are in the same community and -1 if they are not. Thus, this can be maximized if connected nodes fall into the same community and unconnected nodes do not. The modularity is defined as the maximum value of this function across all potential community assignments $\left\{C_{i}\right\}_{i}$, rescaled to fall between -0.5 and 1 [71]

Transitivity The transitivity is the total number of number of 3-way reciprocal connections compared to the total possible number of such connections, i.e.,

$$
\frac{1}{\sum_{i}\left(\sum_{j} A_{i, j}\right)\left(\left(\sum_{j} A_{i, j}\right)-1\right)} \sum_{i, j, k, i \neq j \neq k} A_{i, j} A_{j, k} A_{i, k}
$$

Note that this is distinct from local efficiency because it considers the network as a whole rather than considering each node $i$ individually and then averaging.

In addition to considering these graph metrics, we also consider two nodal graph metrics:

Degree The degree is a nodal metric which measures the total number of edges connected to a node, i.e.,

$$
\operatorname{degree}(i)=\sum_{j} A_{i, j}
$$

Centrality Betweenness centrality is a nodal metric which measures the faction of shortest paths between all pairs of nodes in a network which pass through the given node. It does not have a closed form equation and must be computed using an algorithm [72].

Graph metrics linear model To quantify the impact of SA and TA jointly, we utilized a linear model. We fit the "SA- $\lambda+$ SA- $\infty$ " model

$$
\text { metric }=\beta_{1} \mathrm{SA}-\lambda+\beta_{2} \mathrm{SA}-\infty
$$

or the "All" model

$$
\text { metric }=\beta_{1} \mathrm{SA}-\lambda+\beta_{2} \mathrm{SA}-\infty+\beta_{3} \mathrm{TA}-\Delta_{1}
$$

on $50 \%$ of the data, randomly chosen from all sessions. Shown in Figure 1e and Figure $\mathrm{S} 1$ is the Spearman correlation of the $50 \%$ held-out data with the model fit. Because the fit is on held-out data, the correlation with the linear model does not necessarily need to be higher than the correlation with any of the individual factors in the model.

Generative model The generative model generates timeseries which can be analyzed like rs-fMRI timeseries. It takes two parameters - the noiseless SA- $\lambda$ (SA- $\lambda^{\text {gen }}$, and the noiseless SA- $\infty$ (SA- $\left.\infty^{\text {gen }}\right)$ - and also uses two pieces of information from the data- the TA- $\Delta_{1} \phi_{i}$ from each region $i$, and the Euclidean distance $d_{i, j}$ between the centroids of each pair of regions $i$ and $j$.

The model operates in two basic steps. First, we generate random timeseries which are both spatially and temporally autocorrelated. For this first step, all timeseries will have uniformly high TA, and SA determined by the parameters SA- $\lambda$ and SA- $\infty$. Using the derivation outlined in Supplement 2.1, we generate $N$ high-pass filtered (cutoff frequency $0.01 \mathrm{hz}$, 4 th order Butterworth filter) Brownian noise timeseries (frequency spectrum $1 / f^{2}$ ) of length $T$ such that, for timeseries $x_{i}[t]$ and $x_{j}[t]$, we have

$\mathbb{E}\left(\operatorname{corr}\left(x_{i}[t], x_{j}[t]\right)\right)=\mathrm{SA}-\infty^{\mathrm{gen}}+\left(1-\mathrm{SA}-\infty^{\mathrm{gen}}\right) e^{-d_{i, j} / S A-\lambda^{\mathrm{gen}}}$

where corr is the Pearson correlation, similar to Equation 1. The matrix consisting of all such expected correlations between regions $i$ and $j$ forms the matrix $C_{i, j}$ required by the algorithm described in Supplement 2.1, such that

$$
C_{i, j}=\mathbb{E}\left(\operatorname{corr}\left(x_{i}[t], x_{j}[t]\right)\right)
$$

For non-negative SA- $\infty^{\text {gen }}, C_{i, j}$ will be positive semidefinite.

Second, we reduce the temporal autocorrelation of each timeseries to match that of the original data. Brownian motion timeseries have high TA and adding white noise to a timeseries reduces TA, so we add white noise to each timeseries $x[i]$ until the TA- $\Delta_{1}$ of the timeseries is equal to the empirical TA- $\Delta_{1} \phi_{i}$. Thus, we have to choose a distinct amount of noise to add to each timeseries such that $\operatorname{corr}\left(x_{i}[t], x_{i}[t+1]\right)=\phi_{i}$. We update $x_{i}[t] \leftarrow x_{i}[t]+N\left(0, h\left(\phi_{i}\right)\right)$ for some function $h\left(\phi_{i}\right)$ given by Equation 4 using the analytical derivation outlined in Supplement 2.3. The function $h\left(\phi_{i}\right)$ is defined for $\phi_{i}>0$, and the variance of a random variable must be non-negative, so $\phi_{i}$ is truncated such that $\phi_{i} \geq 0.0001$ and $h\left(\phi_{i}\right) \geq 0$. Note that SA- $\lambda^{\text {gen }}$ and SA- $\infty^{\text {gen }}$ are parameters of the underlying process, and differ from the SA- $\lambda$ and SA- $\infty$ of the generated timeseries due to the addition of noise. The resulting timeseries exhibit a spatial embedding given by the parcel centroid distances $d_{i, j}$ and parameters SA- $\lambda^{\text {gen }}$ and SA- $\infty^{\text {gen }}$, and have regional TA $-\Delta_{1}$ equal to the original timeseries.

Model variants We test several variants of the model to determine the importance of three key components of the model: added uncorrelated noise, TA, and SA.

TA only This model modifies the generative model to remove the spatial embedding. Specifically, it fixes the parameters $\mathrm{SA}-\lambda^{\text {gen }}$ and SA- $\infty^{\text {gen }}$ to 0 , such that $\operatorname{corr}\left(x_{i}[t], x_{j}[t]\right)=I_{i=j}$ where $I$ is the indicator function. It takes no parameters.

SA only We generate random multivariate Gaussian noise with mean zero and covariance matrix

$$
C_{i, j}=\mathrm{SA}-\infty^{\mathrm{gen}}+\left(1-\mathrm{SA}-\infty^{\mathrm{gen}}\right) \exp \left(-d_{i, j} / \mathrm{SA}-\lambda^{\mathrm{gen}}\right)
$$

similar to Equation 1. It takes two parameters, $S A-\lambda{ }^{\text {gen }}$ and $\mathrm{SA}-\infty^{\text {gen }}$. Unlike the generative model, $\mathbb{E}(\mathrm{SA}-\lambda)=\mathrm{SA}-\lambda^{\text {gen }}$ and $\mathbb{E}(\mathrm{SA}-\infty)=\mathrm{SA}-\infty^{\text {gen }}$.

Noiseless In our generative model, we make sure timeseries have the desired TA- $\Delta_{1}$ by generating timeseries with uniformly high TA- $\Delta_{1}$, and then adding different magnitudes of white noise to each timeseries to match TA- $\Delta_{1}$ to the original timeseries. In this model, we do not add white noise to the timeseries. To match TA- $\Delta_{1}$ to the original timeseries, we generate timeseries directly with matched TA- $\Delta_{1}$. This is possible because the derivation presented in Supplement 2.1 does not require each timeseries' power spectrum to be identical. Thus, we achieve this diversity in TA- $\Delta_{1}$ by assuming 
each timeseries has a filtered pink noise $\left(1 / f^{\alpha}\right)$ temporal dynamics. Then, for each timeseries $i$, we find an exponent $\alpha_{i}$ such that the high pass filtered $1 / f^{\alpha_{i}}$ spectrum has expected TA- $\Delta_{1} \phi_{i}$.

Thu success of this procedure requires us to choose $\alpha_{i}$ such that the high pass filtered $1 / f^{\alpha_{i}}$ spectrum has TA- $\Delta_{1}$ equal to $\phi_{i}$. The mapping $\phi_{i} \rightarrow \alpha_{i}$ can be determined by numerically inverting the $\alpha_{i} \rightarrow \phi_{i}$ mapping implied by Supplement 2.2. High pass filtering is performed at the level of the power spectrum by multiplying the square of the amplitude response of the filter by the power spectrum. Additionally, in this model, it is possible to determine the SA- $\lambda^{\text {gen }}$ and SA- ${ }^{\text {gen }}$ parameters directly from the data, without the need to fit parameters, by using the procedure is outlined in Supplement 2.1. As in the "SA only" model, $\mathbb{E}(\mathrm{SA}-\lambda)=\mathrm{SA}-\lambda$ gen and $\mathbb{E}(\mathrm{SA}-\infty)=\mathrm{SA}-\infty^{\text {gen }}$.

Thus, in summary, this model makes the following modifications to the generative model: (1) rather than simulating random walks $\left(1 / f^{\alpha}\right.$ where $\left.\alpha=2\right)$, the spectral exponents $\alpha_{i}$ for each region $i$ are chosen such that the resulting TA- $\Delta_{1}$ is equal to each region's TA- $\Delta_{1}$ value; and (2) no noise is added to the powerlaw timeseries.

Homogeneous TA- $\Delta_{1}$ To allow SA and TA to be independently manipulated, we developed a homogeneous variant of the generative model which treats TA as a parameter. Rather than use TA- $\Delta_{1}$ values computed from the original timeseries, this model uses a single fixed value of TA- $\Delta_{1}$, TA- $\Delta_{1}^{\text {gen }}$, for all regions. For simplicity, we fixed SA- $\infty^{\text {gen }}$ to be the mean SA- $\infty$ across all networks. Thus, the model takes two parameters: TA- $\Delta_{1}^{\text {gen }}$ and SA- $\lambda^{\text {gen }}$.

Null models We also test several popular null models.

Phase randomization The power spectrum amplitude of individual timeseries is preserved, but the phases are randomized (Figure S4). This procedure is described in detail in [73]. A Fourier transform was performed on each region's timeseries. Each element of the complex-valued Fourier transform was randomly rotated on the unit circle, and the inverse transform was performed on the phase-randomized spectra.

Zalesky matching This model matches the mean and the variance of the correlation matrix, and is described in full in [6]. Briefly, it matches the first two moments by iteratively computing correlation matrices from timeseries of different durations with different ground-truth correlations. The process continues until the timeseries duration is found which maximally reproduces the mean and variance of the correlation matrix.

Edge reshuffle We preserve the degree of each node while scrambling the edges (Figure S4). This is accomplished by an iterative algorithm described in detail in [75]. Two edges from a graph were selected at random, and the connections were swapped. This swap was iterated $k$ times, where $k$ was chosen here to be 5 times the number of edges in the network. The result is that each node has the same number of connections, but those connections are randomized.

Models not considered Despite our parameterization based on the TA- $\Delta_{1}$ we do not report on timeseries generated us- ing vector autoregressive models (VAR). These models have two limitations within this context. First, they do not reproduce the observed long-memory processes observed within rs-fMRI timeseries. Second, when fitting data using these models, the TA- $\Delta_{1}$ parameter fits to values very close to 1 , resulting in parameter degeneracy.

Likewise, we did not directly fit the economical clustering model to data. Due to the variability in individual instantiations of this model and the lack of smoothness, they were incompatible with our numerical fitting algorithm, thus preventing the use of comparable methodology to perform the fitting. This, combined with the long execution time of the model, made such individual level fitting infeasible.

Model fitting procedure Due to the fact that uncorrelated random noise was added after the spatial embedding in our model, the SA- $\lambda^{\text {gen }}$ and $\mathrm{SA}-\infty^{\text {gen }}$ parameters in the generative model were not identical to the observed SA- $\lambda$ and $S A-\infty$. We derived a mathematical method for directly matching the generative model's SA- $\lambda^{\text {gen }}$ and SA- $\infty^{\text {gen }}$ parameters to the data without the use of fitting, but technical constraints prevented an implementation of the procedure (Supplement 2.4). Therefore, we could not directly estimate the model based on observed SA- $\lambda$ and SA- $\infty$.

Instead, the generative model's SA- $\lambda^{\text {gen }}$ and $\mathrm{SA}-\infty^{\text {gen }}$ parameters were fit to individual subject FC matrices to match that matrix's eigenvalue distribution. The model was fit using differential evolution [76], a gradient-free global heuristic search method, to the mean squared error between the sorted eigenvalue distributions of the subject and model FC matrices. Eigenvalues were non-negative due to the positivesemidefiniteness of the correlation matrix. The model was implemented in such a way that they preserved smoothness with respect to the parameters, meaning that for a given random seed, small perturbations of the parameters caused only small changes in the timeseries, and hence in the structure of the graph. Optimization was performed on the mean objective function from two random seeds. All reported statistics and metrics about the models come from a single instantiation of the model using a different random seed than either seed used during fitting. Parameters for the "SA only" model were also fit using the same procedure.

Most alternative models had no parameters ("TA only", "Phase randomization", "Zalesky matching", and "Edge reshuffle"). For the "Noiseless" model, parameters could be fit directly using the formalism in Supplement 2.1. This formalism is not guaranteed to converge for all subjects. When the algorithm was unable to produce valid timeseries, we excluded these subjects from the analysis. Results were qualitatively similar when parameters were fit to the eigenvalue distribution as described above which forced parameters into valid regimes.

Economical clustering model Here, we use the term "generative model" to refer to a model which produces a timeseries for each brain region - graphs can then be constructed by processing these timeseries the same way as subjects' rs-fMRI timeseries. By contrast, in the graph theory literature, the term "generative model" usually refers to models which con- 
bioRxiv preprint doi: https://doi.org/10.1101/2021.06.01.446561; this version posted June 1, 2021. The copyright holder for this preprint (which was not certified by peer review) is the author/funder. All rights reserved. No reuse allowed without permission.

Shinn et al.

struct graphs directly through the iterative addition of nodes or edges $[14,15,104]$. The economical clustering (EC) model is one such model which is popular for studying brain networks $[14,15]$ (Supplement 4.1). In this model, connections between nodes are determined by one parameter governing the impact of distance and one for the impact of clustered topology. The probability of an edge forming between two brain regions is proportional to the product of the Euclidean distance between the regions raised to some power (the distance parameter), and the fraction of shared neighbors between them raised to some power (the clustering parameter). Full model details are provided in Supplement 4.2 and in Ref. [14].

To compare the two models, we simulated the EC model across a spectrum of distance and clustering parameters, and then fit a generative model to the simulated networks. Because our generative model takes two SA-related parameters and obtains TA on a regional level directly from the data, we compared the EC model to the homogeneous variant of the generative model, which includes one SA parameter and one TA parameter. We used the EC model to simulate 10 networks per combination of parameters, fitting the homogeneous generative model to each of these 10 networks. Since the EC model produced graphs rather than FC matrices, we could not use our previous approach of fitting by eigenvalues, nor could we derive an analytic approach to parameter estimation. Thus, we fit using the objective function from Ref. [15]. Full details are provided in Supplement 4.3.

Subnetwork classification We define subnetworks of the AAL atlas using the classification in Ref. [58]. Due to the small number of parcels in the insula (2) and central (6) subnetworks defined in Ref. [58], we reclassify the precentral and postcentral gyrus regions from the "central" network into the "frontal" and "parietal" regions, respectively, and exclude the insula and Rolandic operculum regions due to their ambiguous lobe classification. Statistical results are unaffected by this reclassification.

Code availability Source code will be made available upon publication. Code was implemented using the standard Python stack [80,81] and other libraries [82-84]. Source code was checked for correctness using software verification techniques [85].

\section{References}

[51] Glasser, M. F. et al. A multi-modal parcellation of human cerebral cortex. Nature 536, 171-178 (2016).

[52] Robinson, E. C. et al. MSM: A new flexible framework for multimodal surface matching. NeuroImage 100, 414-426 (2014).

[53] Friedman, L., Glover, G. H., Krenz, D. \& Magnotta, V. Reducing interscanner variability of activation in a multicenter fMRI study: Role of smoothness equalization. NeuroImage 32, 1656-1668 (2006).

[54] Scheinost, D., Papademetris, X. \& Constable, R. T. The impact of image smoothness on intrinsic functional connectivity and head motion confounds. NeuroImage 95, 13-21 (2014).

[55] Shen, X., Tokoglu, F., Papademetris, X. \& Constable, R. Groupwise whole-brain parcellation from resting-state fMRI data for network node identification. NeuroImage 82, 403-415 (2013).

[56] Shafto, M. A. et al. The cambridge centre for ageing and neuroscience (cam-CAN) study protocol: a cross-sectional, lifespan, multidisciplinary examination of healthy cognitive ageing. BMC Neurology 14 (2014).
[57] Taylor, J. R. et al. The cambridge centre for ageing and neuroscience (cam-CAN) data repository: Structural and functional MRI, MEG, and cognitive data from a cross-sectional adult lifespan sample. NeuroImage 144, 262-269 (2017).

[58] Tzourio-Mazoyer, N. et al. Automated anatomical labeling of activations in SPM using a macroscopic anatomical parcellation of the MNI MRI single-subject brain. NeuroImage 15, 273-289 (2002).

[59] Bullmore, E. \& Sporns, O. Complex brain networks: graph theoretical analysis of structural and functional systems. Nature Reviews Neuroscience 10, 186-198 (2009).

[60] Fornito, A., Zalesky, A. \& Bullmore, E. Fundamentals of Brain Network Analysis (Academic Press, 2016).

[61] Mantegna, R. Hierarchical structure in financial markets. The European Physical Journal B 11, 193-197 (1999).

[62] Kruskal, J. B. On the shortest spanning subtree of a graph and the traveling salesman problem. Proceedings of the American Mathematical Society 7, 48-48 (1956).

[63] Termenon, M., Jaillard, A., Delon-Martin, C. \& Achard, S. Reliability of graph analysis of resting state fMRI using test-retest dataset from the Human Connectome Project. NeuroImage 142, 172-187 (2016).

[64] Noble, S., Scheinost, D. \& Constable, R. T. A decade of test-retest reliability of functional connectivity: A systematic review and metaanalysis. NeuroImage 203, 116157 (2019).

[65] Finn, E. S. et al. Functional connectome fingerprinting: identifying individuals using patterns of brain connectivity. Nature Neuroscience 18, 1664-1671 (2015).

[66] Noble, S. et al. Influences on the test-retest reliability of functional connectivity MRI and its relationship with behavioral utility. Cerebral Cortex 27, 5415-5429 (2017).

[67] Horien, C. et al. Considering factors affecting the connectome-based identification process: Comment on waller et al. NeuroImage 169, 172175 (2018).

[68] Rubinov, M. \& Sporns, O. Complex network measures of brain connectivity: Uses and interpretations. NeuroImage 52, 1059-1069 (2010).

[69] Newman, M. E. J. Assortative mixing in networks. Physical Review Letters 89 (2002).

[70] Latora, V. \& Marchiori, M. Efficient behavior of small-world networks. Physical Review Letters 87 (2001).

[71] Newman, M. E. J. \& Girvan, M. Finding and evaluating community structure in networks. Physical Review E 69 (2004).

[72] Brandes, U. A faster algorithm for betweenness centrality. The Journal of Mathematical Sociology 25, 163-177 (2001).

[73] Theiler, J., Eubank, S., Longtin, A., Galdrikian, B. \& Farmer, J. D. Testing for nonlinearity in time series: the method of surrogate data. Physica D: Nonlinear Phenomena 58, 77-94 (1992).

[74] Zalesky, A., Fornito, A. \& Bullmore, E. On the use of correlation as a measure of network connectivity. NeuroImage 60, 2096-2106 (2012).

[75] Maslov, S. \& Sneppen, K. Specificity and Stability in Topology of Protein Networks. Science 296, 910-913 (2002).

[76] Storn, R. \& Price, K. Differential evolution - a simple and efficient heuristic for global optimization over continuous spaces. Journal of Global Optimization 11, 341-359 (1997).

[77] Vértes, P. E. et al. Simple models of human brain functional networks. Proceedings of the National Academy of Sciences 109, 58685873 (2012).

[78] Betzel, R. F. et al. Generative models of the human connectome. NeuroImage 124, 1054-1064 (2016).

[79] Bassett, D. S. \& Sporns, O. Network neuroscience. Nature Neuroscience 20, 353-364 (2017).

[80] Harris, C. R. et al. Array programming with NumPy. Nature 585, 357362 (2020).

[81] Virtanen, P. et al. SciPy 1.0: fundamental algorithms for scientific computing in python. Nature Methods 17, 261-272 (2020).

[82] LaPlante, R. A., Douw, L., Tang, W. \& Stufflebeam, S. M. The connectome visualization utility: Software for visualization of human brain networks. PLOS ONE 9, e113838 (2014).

[83] Donges, J. F. et al. Unified functional network and nonlinear time series analysis for complex systems science: The pyunicorn package. Chaos: An Interdisciplinary Journal of Nonlinear Science 25, 113101 (2015).

[84] Vallat, R. Pingouin: statistics in python. Journal of Open Source Software 3, 1026 (2018).

[85] Shinn, M. Refinement type contracts for verification of scientific investigative software. In Chakraborty, S. \& Navas, J. A. (eds.) Verified Software. Theories, Tools, and Experiments, 143-160 (Springer International Publishing, Cham, 2020). 


\section{Supplemental information}

\section{Supplement 1: Relationship between TA- $\Delta_{1}$ and long memory processes}

fMRI timeseries are thought to show characteristics of a long memory process, i.e., time points which are separated by a large lag show a high correlation $[28,33,34]$. These processes are often characterized by their autocorrelation function (ACF). For a timeseries $x$, the autocorrelation function is defined as

$$
A C F_{x}(k)=\operatorname{corr}(x[t], x[t+k])
$$

where corr is the Pearson correlation and $k$ is the lag at which to evaluate the ACF. Long memory processes have an ACF which decays slowly across lags, meaning that relatively high correlations exist even at large lags. However, in this work, we use TA- $\Delta_{1}$ as our measure of temporal autocorrelation, which is identical to the lag-1 term of the ACF, $A C F_{x}(1)$. If fMRI timeseries show long memory properties, and long memory processes are based on many terms of the ACF, this raises the question of whether TA- $\Delta_{1}$ is an appropriate measure to evaluate TA.

We demonstrate that TA- $\Delta_{1}$ is representative of diverse forms of temporal autocorrelation in our data using a three-fold approach. First, we show that TA- $\Delta_{1}$, the first lag term in the ACF, is more reliable than higher lag terms in the ACF. Second, we show that TA- $\Delta_{1}$ is predictive of higher lag terms of the ACF, indicating shared information between the terms. Finally, we fit a parametric model of long memory dynamics to our data, and show a strong correlation between the long memory parameter and TA- $\Delta_{1}$. These results align with previous work showing TA- $\Delta_{1}$ may be representative of higher lag terms of the ACF [28,89-91].

Reliability of the ACF decreases with increasing lag. For each lag $k$, we computed the reliability of the corresponding term in the ACF, $A C F_{x}(k)$. We found that the median reliability across brain regions decreased as $k$ increased, and that TA- $\Delta_{1}$ (i.e., $A C F_{x}(1)$ ) maximized median reliability (Figure S2a,h). To confirm that this occurs for each brain region individually, we found the difference in reliability between the $A C F_{x}(1)$ and $A C F_{x}(k)$, which also decreased across lags (Figure S2b,i). In addition to the reliability of regional TA- $\Delta_{1}$, we are interested in how each subject's mean TA- $\Delta_{1}$ (averaging across regions) compares to the mean across other lags. We found that, like in the regional case, reliability decreased as the lag increased (Figure S2c,j). Thus, the reliability at short compared to long time lags motivates a prioritization for TA- $\Delta_{1}$ over higher lags.

In addition, TA- $\Delta_{1}$ is predictive of higher terms of the ACF. We divided the data into 10 randomly selected subsets for the HCP and Cam-CAN datasets, and 12 subsets for the TRT data. We fit the regression model

$$
A C F_{x}(k)=\beta_{0}+\beta_{1} \mathrm{TA}-\Delta_{1}
$$

to one of these subsets. Then, we evaluated the $R^{2}$ of the model on each of the other subsets. Note that this is a more "difficult" prediction than traditional k-fold cross validation, as it uses less training data to make the predictions. We evaluated this fit on the remaining subsets. Using this procedure, we found that TA- $\Delta_{1}$ was predictive of higher lags of the ACF (Figure S2d,k,o). This indicates that information represented in TA- $\Delta_{1}$ is partially redundant with that in higher lags.

Furthermore, individual variation in long memory properties of our data can be captured by individual variation in TA- $\Delta_{1}$. 
Shinn et al.

We focused our analysis on the fractional integration constant $d$ from an ARFIMA model, frequently used to capture long memory dynamics ${ }^{1}$ [92]. Following previous work, we estimated $d$ through a univariate wavelet-based Whittle estimator $[93,94]$. We found that TA- $\Delta_{1}$ is highly correlated with $d$ when averaged across subjects (Figure S2e,1,p), averaged across regions (Figure S2f,m,q), or without averaging (Figure S2g,n,r). Under specific assumptions, this relationship between $d$ and TA- $\Delta_{1}$ can be described analytically [90,91]. Furthermore, $d$ showed similar reliability to TA- $\Delta_{1}$ (Figure S2a-c,h-j), and TA- $\Delta_{1}$ was predictive of $d$ (Figure S2d,k,o). Therefore, in the datasets considered here, TA- $\Delta_{1}$ is a reliable and effective measure of TA in general, even though it cannot directly measure long memory dynamics.

\footnotetext{
${ }^{1} d$ is closely related to other measures of long memory dynamics such as the Hurst exponent $H$; under specific conditions, $H=d+0.5$ [92].
} 


\section{Supplement 2: Mathematical derivations for the generative model}

\subsection{Constructing timeseries with specified correlations and power spectra}

We seek to create an algorithm that produces timeseries with TA and SA similar to that found in fMRI data. Diverse methods of generating timeseries which exhibit both TA and SA already exist, such as vector autoregressive (VAR) and OrnsteinUhlenbeck processes. These models directly simulate timeseries in the time domain, introducing TA and SA locally. However, prior research demonstrating powerlaw dynamics in fMRI data [28, 33, 34] suggests that VAR and similar models are inadequate for our purposes, as they are unable to model complex power spectral properties. More complex variants of these models, such as VARFI and FIVAR, are able to capture long memory dynamics, but they are unable to simulate from arbitrary power spectra [93]. Instead, we seek to generate power spectra which represent the tradeoff between long-memory dynamics, i.e. filtered $1 / f^{\alpha}$ noise for frequencies above $0.01 \mathrm{hz}$, and optional white noise, with a flat power spectrum.

To introduce SA in our method, we spatially embed these timeseries with a given covariance matrix $C$. In our case, $C$ is given in Equation 2. The usual method of spatially embedding timeseries with a given covariance matrix $C$ is to multiply them by the Cholesky decomposition of $C$. However, this process involves the linear combination of distinct timeseries, which changes their power spectra, destroying the desired power spectrum. To avoid this confound, we introduce the following algorithm, a generalization of Ref. [95], which is able to produce correlated timeseries with arbitrary power spectra. The algorithm can alternatively be seen as a generalization of phase randomization to obtain specified correlational structure.

Algorithm Let $N$ be the number of desired timeseries, and let $T$ be the even length of the desired timeseries. Given an $N \times N$ correlation matrix $C_{i, j}$ and a set of exponents $\alpha_{i}$, we seek to generate a set of timeseries $\left\{x_{1}[t], \ldots, x_{N}[t]\right\}$ such that each timeseries $x_{i}[t]$ has a specified estimate of the power spectrum $\left|X_{i}[k]\right|^{2}$ and the correlation matrix of the resulting timeseries has expected value $C_{i, j}$. The following algorithm operates by generating a set of complex frequency-domain coefficients in the domain $[-T / 2+1, T / 2]$, introducing SA and TA independently, and then performing an inverse discrete Fourier transform to create the desired set of timeseries.

1. Pick the desired power spectra estimates $\left\{\left|X_{1}[k]\right|^{2}, \ldots,\left|X_{N}[k]\right|^{2}\right\}$, representing the desired temporal structure, and $N \times N$ correlation matrix $C$, representing the desired spatial structure. These choices must satisfy the requirement that the matrix

$$
\Sigma_{i, j}=\frac{C_{i, j}}{\operatorname{sim}\left(\left|X_{i}[k]\right|,\left|X_{j}[k]\right|\right)}
$$

is positive semi-definite, where sim denotes cosine similarity, i.e.,

$$
\operatorname{sim}\left(\left|X_{i}[k]\right|,\left|X_{j}[k]\right|\right)=\frac{\sum_{k}\left|X_{i}[k]\right|\left|X_{j}[k]\right|}{\sqrt{\sum_{k}\left|X_{i}[k]\right|^{2}} \sqrt{\sum_{k}\left|X_{j}[k]\right|^{2}}} .
$$

2. For each region $n \in\{1, \ldots, N\}$, and for each frequency $k$ in the positive sub-Nyquist Fourier domain, $1 \leq k \leq T / 2$, sample two vectors from a multivariate normal distribution $a_{n}^{R}[k], a_{n}^{I}[k] \sim N(0, \Sigma)$.

3. Set $a_{n}^{I}[T / 2]=0$ for all $n$. Because $T$ is even, by way of symmetry the frequency-domain coefficients of the Nyquist 
Shinn et al.

frequency must be real. Set $a_{n}^{I}[0]=0$ so that the resulting timeseries will be real.

4. Form the complex frequency-domain coefficients by letting $c_{n}[k]=\left|X_{i}[k]\right|\left(a_{n}^{R}[k]+i a_{n}^{I}[k]\right)$ for $0 \leq k \leq T / 2$, and $c_{n}[-k]=\overline{c_{n}[k]}$ for $0<k<T / 2$.

5. Perform an inverse discrete Fourier transform for each $c_{n}[k]$ to obtain the timeseries $x_{n}[t]$.

Each of the resulting timeseries $x_{i}[t]$ will have a frequency spectrum approximately equal to $\left|X_{i}[k]\left(a_{n}^{R}[k]+i a_{n}^{I}[k]\right)\right|^{2}=\left|X_{i}\right|^{2}$, the desired temporal structure. To show that $\left\{x_{1}[t], \ldots, x_{N}[t]\right\}$ have the desired spatial structure, we show that for any $i, j$, $\mathbb{E}\left[\operatorname{corr}\left(x_{i}[t], x_{j}[t]\right)\right]=C_{i, j}$. As an intermediate step, we show that $\left.\mathbb{E}\left[\operatorname{corr}\left(x_{i}[t], x_{j}[t]\right)\right]=\operatorname{corr}\left(\operatorname{Re}\left(X_{i}[k]\right), \operatorname{Re}\left(X_{j}[k]\right)\right)\right)$.

Let $x_{i}[t]$ and $x_{j}[t]$ be two of the timeseries resulting from this algorithm. Note that, for large $N, \operatorname{corr}\left(x_{i}[t], x_{j}[t]\right)=$ $\operatorname{cov}\left(x_{i}[t], x_{j}[t]\right) /\left(\operatorname{var}\left(x_{i}[t]\right) \cdot \operatorname{var}\left(x_{j}[t]\right)\right)^{1 / 2}$, where var and cov are the empirical estimates of the variance and covariance, respectively. We first seek to expand $\operatorname{cov}\left(x_{i}[t], x_{j}[t]\right), \operatorname{var}\left(x_{i}[t]\right)$, and $\operatorname{var}\left(x_{j}[t]\right)$. Without loss of generality, we may assume that $x_{i}[t]$ and $x_{j}[t]$ have a mean of 0 , as their mean does not influence the spatial or temporal structure of the set of timeseries, so we can estimate $\operatorname{cov}\left(x_{i}[t], x_{j}[t]\right)=1 / N \sum_{t} x_{i}[t] x_{j}[t]$.

Recall that $X_{i}[k]$ is the discrete Fourier transform $\mathcal{F}\left(x_{i}[t]\right)$. We denote the even and odd components $x_{i}^{e}[t]=\mathcal{F}^{-1}(\operatorname{Re}(X[k])$ and $x_{i}^{o}[t]=\mathcal{F}^{-1}(i \operatorname{Im}(X[k])$. Thus, our estimator of the covariance can be written as

$$
\operatorname{cov}\left(x_{i}[t], x_{j}[t]\right)=1 / N\left(\sum_{t} x_{i}^{e}[t] x_{j}^{e}[t]+\sum_{t} x_{i}^{e}[t] x_{j}^{o}[t]+\sum_{t} x_{i}^{o}[t] x_{j}^{e}[t]+\sum_{t} x_{i}^{o}[t] x_{j}^{o}[t]\right)
$$

For the even part of the function, $\sum_{t} x_{i}^{e}[t] x_{j}^{e}[t]=\sum_{t} x_{i}^{e}[t] \overline{x_{j}^{e}[t]}$, and by Parseval's theorem, this equals $1 / N \sum_{k} \operatorname{Re}\left(X_{i}[k] \overline{\operatorname{Re}\left(X_{j}[k]\right)}\right.$. This can be written as $1 / N \sum_{k} \operatorname{Re}\left(X_{i}[k]\right) \operatorname{Re}\left(X_{j}[k]\right)=\operatorname{cov}\left(X_{i}[k], X_{j}[k]\right)$. By the same logic, $\sum_{t} x_{i}^{o}[t] x_{j}^{o}[t]=\operatorname{cov}\left(\operatorname{Re}\left(X_{i}[k]\right), \operatorname{Re}\left(X_{j}[k]\right)\right)$.

The real and imaginary components of $X_{i}[k]$ are independent by construction, which means $\mathbb{E}\left[\sum_{t} x_{i}^{e}[t] x_{j}^{o}[t]\right]=0$ and $\mathbb{E}\left[\sum_{t} x_{i}^{o}[t] x_{j}^{e}[t]\right]=0$. Thus, we can rewrite the covariance estimator as

$$
\begin{aligned}
\mathbb{E}\left[\operatorname{cov}\left(x_{i}, x_{j}\right)\right] & =1 / N\left(\operatorname{cov}\left(\operatorname{Re}\left(X_{i}[k]\right), \operatorname{Re}\left(X_{j}[k]\right)\right)+0+0+\operatorname{cov}\left(\operatorname{Re}\left(X_{i}[k]\right), \operatorname{Re}\left(X_{j}[k]\right)\right)\right) \\
& =2 \cdot \operatorname{cov}\left(\operatorname{Re}\left(X_{i}[k]\right), \operatorname{Re}\left(X_{j}[k]\right)\right) / N .
\end{aligned}
$$

This gives $\mathbb{E}\left[\operatorname{var}\left(x_{i}\right)\right]=\mathbb{E}\left[\operatorname{cov}\left(x_{i}, x_{i}\right)\right]=2 \cdot \operatorname{var}\left(\operatorname{Re}\left(X_{i}[k]\right)\right) / N$, so

$$
\begin{aligned}
\mathbb{E}\left[\operatorname{corr}\left(x_{i}[k], x_{j}[k]\right)\right] & =\frac{2 \cdot \operatorname{cov}\left(\operatorname{Re}\left(X_{i}[k]\right), \operatorname{Re}\left(X_{j}[k]\right)\right) / N}{\sqrt{2 \cdot \operatorname{var}\left(\operatorname{Re}\left(X_{i}[k]\right)\right) / N} \cdot \sqrt{2 \cdot \operatorname{var}\left(\operatorname{Re}\left(X_{j}[k]\right)\right) / N}} \\
& =\frac{\operatorname{cov}\left(\operatorname{Re}\left(X_{i}[k]\right), \operatorname{Re}\left(X_{j}[k]\right)\right)}{\sqrt{\operatorname{var}\left(\operatorname{Re}\left(X_{i}[k]\right)\right)} \cdot \sqrt{\operatorname{var}\left(\operatorname{Re}\left(X_{j}[k]\right)\right.}} \\
& =\operatorname{corr}\left(\operatorname{Re}\left(X_{i}[k]\right), \operatorname{Re}\left(X_{j}[k]\right)\right)
\end{aligned}
$$

as desired. A similar procedure applies to the imaginary component of $X_{i}[k]$.

We now show that $\mathbb{E}\left[\operatorname{corr}\left(\operatorname{Re}\left(X_{i}[k]\right), \operatorname{Re}\left(X_{j}[k]\right)\right)\right]=C_{i, j}$. We note that, by construction, $a_{i}^{R}[k]$ and $\left|X_{i}[k]\right|^{2}$ are independent, and that $\mathbb{E}\left(a_{i}^{R}[k]\right)=0$, meaning $\left.\mathbb{E}\left[\operatorname{Re}\left(X_{i}[k]\right)\right]=\mathbb{E}\left[\left|X_{i}[k]\right| a_{i}^{R}[k]\right)\right]=\mathbb{E}\left[\left|X_{i}[k]\right|\right] \cdot \mathbb{E}\left[a_{i}^{R}[k]\right]=0$. This allows us to expand as 
before, giving

$$
\mathbb{E}\left[\operatorname{corr}\left(\operatorname{Re}\left(X_{i}[k]\right), \operatorname{Re}\left(X_{j}[k]\right)\right)\right]=\frac{\mathbb{E}\left[\sum_{k}\left(\left|X_{i}[k]\right|\left|X_{j}[k]\right|\right) a_{i}^{R}[k] a_{j}^{R}[k]\right]}{\mathbb{E}\left[\sum_{k}\left(\left|X_{i}[k]\right| a_{i}^{R}[k]\right)^{2}\right]^{1 / 2} \cdot \mathbb{E}\left[\sum_{k}\left(\left|X_{j}[k]\right| a_{j}^{R}[k]\right)^{2}\right]^{1 / 2}}
$$

Because $a_{i}^{R}[k]$ and $a_{j}^{R}[k]$ have mean 0 and variance 1 and have correlation equal to approximately $\Sigma_{i, j}$, the product $a_{i}^{R}[k] a_{j}^{R}[k]$ has an expected value of $\Sigma_{i, j}$. By construction, $\left(a_{i}^{R}[k] a_{j}^{R}[k]\right)$ and $\left(\left|X_{i}[k]\right|\left|X_{j}[k]\right|\right)$ are independent, so

$$
\mathbb{E}\left[\sum_{k}\left|X_{i}[k]\right|\left|X_{j}[k]\right| a_{i}^{R}[k] a_{j}^{R}[k]\right]=\Sigma_{i, j} \cdot \mathbb{E}\left[\sum_{k}\left|X_{i}[k]\right|\left|X_{j}[k]\right|\right]=\Sigma_{i, j} \cdot \sum_{k}\left|X_{i}[k]\right|\left|X_{j}[k]\right|
$$

When $i=j, C_{i, j}=1$. By the same logic,

$$
\mathbb{E}\left[\sum_{k}\left(\left|X_{i}[k]\right| a_{i}^{R}[k]\right)^{2}\right]^{1 / 2} \cdot \mathbb{E}\left[\sum_{k}\left(\left|X_{j}[k]\right| a_{j}^{R}[k]\right)^{2}\right]^{1 / 2}=\left(\sum_{k}\left|X_{i}[k]\right|^{2}\right)^{1 / 2} \cdot\left(\sum_{k}\left|X_{j}[k]\right|^{2}\right)^{1 / 2}
$$

and so

$$
\begin{aligned}
\mathbb{E}\left[\operatorname{corr}\left(x_{i}, x_{j}\right)\right] & =\Sigma_{i, j} \cdot \frac{\sum_{k}\left|X_{i}[k]\right|\left|X_{j}[k]\right|}{\left(\sum_{k}\left|X_{i}[k]\right|^{2}\right)^{1 / 2} \cdot\left(\sum_{k}\left|X_{j}[k]\right|^{2}\right)^{1 / 2}} \\
& =\Sigma_{i, j} \cdot \operatorname{sim}\left(\left|X_{i}[k]\right|,\left|X_{j}[k]\right|\right) \\
& =C_{i, j}
\end{aligned}
$$

as desired.

\subsection{Relationship between TA- $\Delta_{1}$ and power spectrum}

Our primary measure of temporal autocorrelation is TA- $\Delta_{1}$, despite the fact that our model uses timeseries with complicated power spectra. To demonstrate that TA- $\Delta_{1}$ can capture important information about the power spectrum, we derive the relationship between the Fourier spectrum and TA- $\Delta_{1}$.

Suppose we have a finite timeseries $x[t]$ with discrete Fourier transform $X[k]$. We claim that its TA- $\Delta_{1}$ is

$$
\operatorname{corr}(x[t], x[t+1])=\frac{1}{\sum_{k=1}^{N-1}|X[k]|^{2}} \sum_{k=1}^{N-1}|X[k]|^{2} \cos (2 \pi k / N)
$$

Let $A C F(x)[\tau]$ denote the autocovariance of $X$ at lag $\tau$, i.e. $\operatorname{cov}(x[t], x[t+\tau])$. By the Wiener-Khinchin theorem, $A C F(x)[\tau]$ is the inverse Fourier transform of an estimate of the power spectrum, $|X[k]|^{2}$. Without loss of generality, let us suppose $x[t]$ 
Shinn et al.

has zero mean, and hence, $X[0]=0$, so that

$$
A C F(x)[\tau]=\sum_{k=1}^{N-1}|X[k]|^{2} e^{i 2 \pi k \tau / N}
$$

Because $x[t]$ is real, $e^{i 2 \pi k \tau / N}=\cos (2 \pi k \tau / N)$. Making the approximation that $\operatorname{var}(x[t])=\operatorname{var}(x[t+1])$, we have

$$
\begin{aligned}
\operatorname{corr}(x[t], x[t+1]) & =\frac{\operatorname{cov}(x[t], x[t+1])}{\sqrt{\operatorname{var}(x[t]) \cdot \operatorname{var}(x[t+1])}} \\
& =\frac{\operatorname{ACF}(x)[1]}{\sum_{k=1}^{N-1}|X[t]|^{2}} \\
& =\frac{1}{\sum_{k=1}^{N-1}|X[t]|^{2}} \sum_{k=1}^{N-1}|X[k]|^{2} \cos (2 \pi k / N)
\end{aligned}
$$

as desired.

\subsection{Relationship between TA- $\Delta_{1}$ and noisy power spectrum}

Additionally, we would like to know how the addition of white noise to a power spectrum will change the TA- $\Delta_{1}$. We prove that, for an finite timeseries $y[t]=x[t]+w[t]$ where $w[t] \sim N(0, \sigma)$ of length $N$,

$$
\mathbb{E}[\operatorname{corr}(y[t], y[t+1])]=\frac{1}{N \sigma^{2}+\sum_{k=1}^{N-1}|X[k]|^{2}} \sum_{k=1}^{N-1}|X[k]|^{2} \cos (2 \pi k / N) .
$$

The Fourier transform is linear, so we can write

$$
\begin{aligned}
|Y[k]|^{2} & =|X[k]+W[k]|^{2} \\
& =|X[k]|^{2}+|W[k]|^{2}+2|X[k]||W[k]| \cos (\angle X[k]-\angle W[k]) .
\end{aligned}
$$

Since $w[t]$ is white noise, $W[k]$ has the properties $\mathbb{E}[|W[k]|]=\sigma \sqrt{N}$ and $\angle W[k] \sim U(0,2 \pi)$. Thus, $\mathbb{E}[\cos (\angle X[t]-\angle W[t])]=0$, so

$$
\begin{aligned}
\mathbb{E}\left[|Y[k]|^{2}\right] & =|X[k]|^{2}+\mathbb{E}\left[|W[k]|^{2}\right]+2|X[k]||W[k]| \mathbb{E}[\cos (\angle X[k]-\angle W[k])] \\
& =|X[k]|^{2}+\sigma^{2} N+0 .
\end{aligned}
$$


Applying our result from Supplement 2.2, we see that

$$
\begin{aligned}
\mathbb{E}[\operatorname{corr}(y[t], y[t+1])] & =\frac{1}{N \sigma^{2}+\sum_{k=1}^{N-1}|X[k]|^{2}} \sum_{k=1}^{N-1}\left(|X[k]|^{2}+N \sigma^{2}\right) \cos (2 \pi k / N) \\
& =\frac{1}{N \sigma^{2}+\sum_{k=1}^{N-1}|X[k]|^{2}} \cdot\left[N \sigma^{2} \sum_{n=1}^{N-1} \cos (2 \pi k / N)+\sum_{n=1}^{N-1}|X[k]|^{2} \cos (2 \pi n / N)\right] \\
& =0+\frac{1}{N \sigma^{2}+\sum_{k=1}^{N-1}|X[k]|^{2}} \sum_{k=1}^{N-1}|X[k]|^{2} \cos (2 \pi k / N) .
\end{aligned}
$$

as desired.

It directly follows that, for a given $\mathbb{E}[\operatorname{corr}(y[t], y[t+1])]=\phi$, we can solve this equation for $\sigma^{2}$ as

$$
\sigma^{2}=\frac{1}{N^{2} \phi} \sum_{k=1}^{N-1}|X[k]|^{2}(\cos (2 \pi k / N)-\phi) .
$$

\subsection{Parameter-free fitting of the generative model}

In theory, it is possible to fit the generative model in a parameter-free manner. In other words, the SA- $\lambda$, SA- $\infty$, and TA- $\Delta_{1}$ statistics can all be measured from the data, and therefore, it should be possible to choose SA- $\lambda^{\text {gen }}$ and SA- $\infty^{\text {gen }}$, with arbitrary power spectra estimators $\left|X_{i}[k]\right|^{2}$ for region $i$, such that the generative model produces timeseries which have a given SA- $\lambda$, SA- $\infty$, and TA- $\Delta_{1}$. We describe the mathematical derivation of a procedure for selecting SA- $\lambda$ and SA- $\infty$ using this logic, and show why this procedure is infeasible in practice.

As a reminder, the generative model has four steps: (1) use SA- $\lambda^{\text {gen }}$ and SA- $\infty^{\text {gen }}$ in conjunction with the nodal Euclidean distances $d_{i, j}$ to generate a spatial correlation matrix $C$; (2) construct a power spectrum corresponding to high-pass filtered Brownian $\left(1 / f^{2}\right)$ noise; (3) construct timeseries $x_{1}, \ldots, x_{N}$ for brain regions $1, \ldots, N$ according to Supplement 2.1 such that $\operatorname{corr}\left(x_{i}, x_{j}\right)=C_{i, j}$ and all timeseries have the power spectrum specified in step (2); (4) for each time point $t$ in each timeseries $x_{i}[t]$, add white noise $w_{i}[t] \sim N\left(0, \sigma_{i}^{2}\right)$, with $\sigma_{i}^{2}$ chosen according to Supplement 2.3 such that $x_{i}[t]$ has TA- $\Delta_{1}$ constant $\mathrm{TA}-\Delta_{1 i}$

To perform the derivation, suppose that we have two timeseries $x_{i}$ and $x_{j}, i \neq j$, such that $x_{i}^{\prime}[t]=x_{i}[t]+w_{i}[t]$, where $x_{i}[t]$ has some power spectrum $\left|X_{i}[t]\right|^{2}$ for all $i$ and $w_{i}[t] \sim N\left(0, \sigma_{i}^{2}\right)$ for given $\sigma_{i}^{2}$. Let the correlation corr $\left(x_{i}, x_{j}\right)=C_{i, j}$ for some unknown $C_{i, j}$. We derive an equation to select $C_{i, j}$ such that $\mathbb{E}\left(\operatorname{corr}\left(x_{i}^{\prime}, x_{j}^{\prime}\right)\right)=C_{i, j}^{\prime}$ for some desired $C_{i, j}^{\prime}$

To begin, we rewrite

$$
\begin{aligned}
& \operatorname{corr}\left(x_{i}, x_{j}\right)=\frac{\operatorname{cov}\left(x_{i}, x_{j}\right)}{\sqrt{\operatorname{var}\left(x_{i}\right) \operatorname{var}\left(x_{j}\right)}} \\
& \operatorname{corr}\left(x_{i}^{\prime}, x_{j}^{\prime}\right)=\frac{\operatorname{cov}\left(x_{i}^{\prime}, x_{j}^{\prime}\right)}{\sqrt{\operatorname{var}\left(x_{i}^{\prime}\right) \operatorname{var}\left(x_{j}^{\prime}\right)}}
\end{aligned}
$$

Since $\mathbb{E}\left(\operatorname{cov}\left(x_{i}+w_{i}, x_{j}+w_{j}\right)\right)=\operatorname{cov}\left(x_{i}, x_{j}\right)$ for $i \neq j$, and $\mathbb{E}\left(\operatorname{var}\left(x_{i}+w_{i}\right)\right)=\operatorname{var}\left(x_{i}\right)+\sigma_{i}^{2}$, we can write 
Shinn et al.

$$
\begin{aligned}
\mathbb{E}\left(\operatorname{corr}\left(x_{i}^{\prime}, x_{j}^{\prime}\right)\right) & =\frac{\operatorname{cov}\left(x_{i}, x_{j}\right)}{\sqrt{\left(\operatorname{var}\left(x_{i}\right)+\sigma_{i}^{2}\right)\left(\operatorname{var}\left(x_{j}\right)+\sigma_{j}^{2}\right)}} \\
& =\frac{\operatorname{corr}\left(x_{i}, x_{j}\right) \sqrt{\operatorname{var}\left(x_{i}\right) \operatorname{var}\left(x_{j}\right)}}{\sqrt{\left(\operatorname{var}\left(x_{i}\right)+\sigma_{i}^{2}\right)\left(\operatorname{var}\left(x_{j}\right)+\sigma_{j}^{2}\right)}} \\
& =\frac{\operatorname{corr}\left(x_{i}, x_{j}\right)}{\sqrt{\left(1+\frac{\sigma_{i}^{2}}{\operatorname{var}\left(x_{i}\right)}\right)\left(1+\frac{\sigma_{j}^{2}}{\operatorname{var}\left(x_{j}\right)}\right)}}
\end{aligned}
$$

The variance of a timeseries $x_{i}$ can be estimated by its power spectrum using Parseval's theorem, such that $v a r\left(x_{i}\right)=$ $\sum_{k=1}^{N / 2}\left|X_{i}[k]\right|^{2} /(N / 2)^{2}$. Thus, substituting $C_{i, j}^{\prime}=\operatorname{corr}\left(x_{i}^{\prime}, x_{j}^{\prime}\right)$ and $C_{i, j}=\operatorname{corr}\left(x_{i}, x_{j}\right)$ and rearranging terms, we have

$$
\begin{aligned}
C_{i, j} & =C_{i, j}^{\prime} \sqrt{\left(1+\frac{\sigma_{i}^{2}}{\operatorname{var}\left(x_{i}\right)}\right)\left(1+\frac{\sigma_{j}^{2}}{\operatorname{var}\left(x_{j}\right)}\right)} \\
& =C_{i, j}^{\prime} \sqrt{\left(1+\frac{\sigma_{i}^{2}(N / 2)^{2}}{\sum_{k=1}^{N / 2}\left|X_{i}[k]\right|^{2}}\right)\left(1+\frac{\sigma_{j}^{2}(N / 2)^{2}}{\sum_{k=1}^{N / 2}\left|X_{j}[k]\right|^{2}}\right)}
\end{aligned}
$$

Thus, we have derived a $C_{i, j}$ which can be used in our procedure to produce timeseries with correlation $C_{i, j}^{\prime}$.

While mathematically interesting, the derivation is not useful in practice. Because we are computing a correlation, $C_{i, j}$ has an upper bound of 1 , thereby bounding the potential values of $C_{i, j}^{\prime}$ which can be produced. In practice this constraint is difficult to satisfy. In our data, $\sigma_{i}^{2}$ can take on values up to an order of magnitude larger than var( $\left.x_{i}\right)$, meaning that only very small values of $C_{i, j}^{\prime}$ (approximately $C_{i, j}^{\prime}<0.1$ ) can satisfy the constraint. Additionally, in the analysis of neural data, we consider more than two timeseries. This means that $C$ must be a correlation matrix, and thus $C$ must be positive semi-definite, or equivalently, have all non-negative eigenvalues. In our data, approximately $25 \%$ of eigenvalues are negative after applying this procedure, showing that it is unlikely to be useful in practice. 


\section{Supplement 3: Relationship between temporal autocorrelation and variance in correlation}

Previous work established that temporal autocorrelation (TA) increases the variance of the Pearson correlation between pairs of timeseries [35, 96-98]. Here, we quantified TA using TA- $\Delta_{1}$, the TA at a single lag. We did this with the understanding that TA- $\Delta_{1}$ correlates with TA at higher lags, or with an underlying long memory process (Supplement 1). Since higher lag TA may have an impact on the variance, it is insufficient to solely measure the effect of TA- $\Delta_{1}$ on the variance in Pearson correlation. A more general approach is to quantify the effect of the entire power spectrum on the variance in Pearson correlation (Supplement 2.2). The power spectrum is a full-rank linear transformation of the autocorrelation function, and therefore, is a non-parametric analysis of TA for both short and long memory dynamics. Thus, we seek to understand the impact of an arbitrary power spectrum on the variance in Pearson correlation of two timeseries.

We focus on a specific case of particular importance by analyzing two independent timeseries with identical power spectra and phases distributed randomly around the unit circle. We show that the variance of the Pearson correlation between these two timeseries increases as the uniformity of the power spectrum decreases. In other words, Pearson correlation is lowest for a timeseries of iid white noise, which by definition has no TA. It increases as the power spectrum deviates from uniformity, and is highest for spectra consisting of a single frequency, i.e. a sinusoid.

The variance of a timeseries is closely connected with the power spectrum. Consider the real, discrete, finite timeseries $x_{1}[t]$ and $x_{2}[t]$ of length $N$, with Fourier transform $X_{1}[k]$ and $X_{2}[k]$ and variance $\sigma_{1}^{2}$ and $\sigma_{2}^{2}$. Without loss of generality, assume $x_{1}[t]$ and $x_{2}[t]$ have mean 0 . We can estimate the covariance for large $N$ as

$$
\operatorname{cov}\left(x_{1}, x_{2}\right)=\frac{1}{N} \sum_{t=0}^{N} x_{1}[t] x_{2}[t]
$$

Then, by Parseval's theorem, we can write the covariance as

$$
\operatorname{cov}\left(x_{1}[t], x_{2}[t]\right)=\frac{1}{N^{2}} \sum_{k=1}^{N}\left|X_{1}[k] \overline{X_{2}[k]}\right| .
$$

Therefore, by noting that the variance of a timeseries is simply self-covariance, the empirical Pearson correlation corr can be written as

$$
\begin{aligned}
\operatorname{corr}\left(x_{1}[t], x_{2}[t]\right) & =\frac{\frac{1}{N^{2}} \sum_{k}\left|X_{1}[k] \overline{X_{2}[k]}\right|}{\sqrt{\frac{1}{N^{2}} \sum_{k}\left|X_{1}[k]\right|^{2}} \sqrt{\frac{1}{N^{2}} \sum_{k}\left|X_{2}[k]\right|^{2}}} \\
& =\frac{1}{\sigma_{1} \sigma_{2} N^{2}} \sum_{k}\left|X_{1}[k] \overline{X_{2}[k]}\right| \\
& =\frac{1}{\sigma_{1} \sigma_{2} N^{2}} \sum_{k=1}^{N}\left|X_{1}[k]\right|\left|X_{2}[k]\right| \cos \left(\theta_{k}\right) .
\end{aligned}
$$

Note that the quantities $\left|X_{1}[k]\right|^{2}$ and $\left|X_{2}[k]\right|^{2}$ are estimates of the power spectra of $x_{1}[t]$ and $x_{2}[t]$. Since we assume the angles 
Shinn et al.

$\theta_{k}$ are selected randomly from the uniform distribution from $[0,2 \pi]$, we can easily compute

$$
\begin{aligned}
\mathbb{E}\left(\operatorname{corr}\left(x_{1}[t], x_{2}[t]\right)\right) & =0 \\
\operatorname{var}\left(\operatorname{corr}\left(x_{1}[t], x_{2}[t]\right)\right) & =\frac{1}{2 \sigma_{1} \sigma_{2}} \sum_{k=1}^{N} \sqrt{\left|X_{1}[k]\right|^{2}\left|X_{2}[k]\right|^{2}}
\end{aligned}
$$

The variance given in Equation 5 is maximized at $1 / 2 \sigma_{1} \sigma_{2}$ when both power spectra have unit spikes at the same location, corresponding to sinusoids with identical frequency. Since we assume that $x_{1}[t]$ and $x_{2}[t]$ have identical power spectra, i.e. $\left|X_{1}[k]\right|^{2}=\left|X_{2}[k]\right|^{2}$, Equation 5 is minimized at $1 / 2 \sigma_{1} \sigma_{2} N$ when $x_{1}[t]$ and $x_{2}[t]$ are white noise, i.e. $\left|X_{1}[k]\right|=1 / N$. (If we had not assumed the power spectra were identical, the variance would be minimized at zero for any non-overlapping power spectra, indicating that it is impossible for these timeseries to show a non-zero Pearson correlation.) Hence, any deviation from a uniformly-distributed power spectrum, as occurs in temporally-autocorrelated timeseries, will increase the variance in the Pearson correlation. 


\section{Supplement 4: Economic clustering model}

\subsection{Overview}

Our analysis focused on generative models at the level of time series. However, another popular type of generative model simulates network formation by directly adding edges to a set of nodes. We refer to these models as "generative graph models" to distinguish them from timeseries-based generative models. Generative graph models have been especially useful in modeling real-world networks $[100,101]$. For example, a generative graph model can capture the property that nodes which are already well-connected are likely to gain even more connections, i.e., that the "rich get richer" [1]. It effectively models the generative process of real-world networks such as social networks, where people with a large number of friends are more likely to gain new friends over time. By simulating how real networks are formed, generative graph models reveal the mechanisms that drive the high-level structure of those networks. For this reason, these models have quickly become ubiquitous in the natural and social sciences.

Brain networks share many properties with other complex biological and physical systems, so they can be analyzed using similar graph-theoretic methods. Recently, generative graph models have been used in neuroscience to model the human connectome $[3,104,105]$, opening up new avenues of analysis of both functional $[13,14]$ and structural $[15,108,109,111,112]$ connectivity data. One reason why generative graph models are particularly attractive in the field is that they appear, at first glance, to model the trade-off between wiring cost and topological efficiency in brain networks [113]. In particular, generative graph models that construct networks based on the physical distance between brain regions and a few basic topological properties of the network have been especially effective $[14,15]$. In addition, generative graph models are relatively simple to construct and conceptualize, and they can be analyzed using computational tools from network science and graph theory.

Despite their popularity and conceptual simplicity, most generative graph models are removed from the underlying biological processes that occur in the brain. This is because the generative process used to construct those models is starkly different from the actual pipeline used to extract connectivity data from brain signals. Nevertheless, these models, such as the economical clustering (EC) model [14], are exceedingly effective at explaining the topology of brain connectivity.

In order to explain this effectiveness using a more biologically-motivated timeseries-based generative process, we compare the EC model from Ref. [14] directly to our generative model. The EC model is controlled by two parameters: one controls the connection probability with respect to distance, and the other with respect to homophily (i.e. shared neighbors, or clustering) between nodes [14]. There are a few apparent symmetries between the EC model and our generative model. Both models produce realistic synthetic networks, and they are each driven by two features, one of which relates connectivity with physical distance. These initial similarities suggest the possibility that the second parameter in each model may also capture similar underlying properties. In fact, our findings show that both pairs of parameters in the two models are indeed tightly correlated, which opens up a new biologically-motivated interpretation of the generative graph model.

\subsection{Economic clustering model}

We compared the generative model to the "economic clustering" (EC) model from Ref. [14], which was chosen because of its good performance and its apparent parallels to our timeseries-based model. We briefly summarize the model here. We constructed a minimum spanning tree of the mean FC matrix using Kruskal's algorithm [114] to ensure that our procedure does 
Shinn et al.

not produce disconnected components. Then, edges were added one at a time according to a probabilistic wiring rule, until the number of edges in the model network and observed network were equal. The relative probability of adding a connection between node $u$ and node $v$ was determined by:

$$
P(u, v) \propto d(u, v)^{\eta} \times K(u, v)^{\gamma}
$$

where $d(u, v)$ is the Euclidean distance between nodes $u$ and $v, K(u, v)$ is the number of shared neighbors between nodes $u$ and $v$, described below, and $\eta$ (the distance parameter) and $\gamma$ (the clustering parameter) are free parameters. The exponent $\eta$ controls the extent to which distance impacts connection probability. For highly negative $\eta$, short-range connections are much more likely; for values of $\eta$ close to 0 , all connections regardless of distance are almost equally likely to be added.

$K(u, v)$ represents the number of shared neighbors between nodes $u$ and $v$, or equivalently, the number of nodes that are connected to both node $u$ and node $v$. We can compute this as $K(u, v)=\sum_{w} A_{u w} A_{w v}$ where $A$ is the adjacency matrix of the graph, i.e. $A_{i j}=1$ if nodes $i$ and $j$ are connected, and $A_{i j}=0$ otherwise. Note that $K(u, v)$ must be recomputed at each iteration of the algorithm, since the number of shared neighbors can change after an edge is added. The exponent $\gamma$ controls the extent to which the number of shared neighbors impacts connection probability. For $\gamma<0$, nodes with few shared neighbors are more likely to be connected; for $\gamma>0$, nodes with many shared neighbors are more likely to be connected.

Note that this procedure does not generate networks which vary smoothly with the parameters $\eta$ and $\gamma$. In other words, for a given seed, a small change in either of these parameters could cause major changes in the topology of the generated graph. This occurs because each edge addition causes a cascading change in connection probabilities, and thus, even slight differences in the first edges added will have a large impact on the generated graph.

It has been shown that generative graph models which only specify connection probability as a function of distance do not capture features such as small-worldness, modularity, and degree distribution of real brain networks; rather, a second parameter is necessary to emulate these topological properties [14]. These findings are consistent with the results of our generative model. In particular, our generative model has two main distinguishing features: spatial autocorrelation and temporal autocorrelation. This apparent symmetry between the generative model and the EC model motivates our comparison of the underlying parameters across the two models.

\subsection{Fitting the generative model to the EC model}

To determine whether the parameters of the generative model and the EC model are correlated, we developed a fitting procedure linking the two models. Because the model is determined by probabilistic wiring rules, a small perturbation in a model parameter may cause a large change in graph structure. Thus, the highly stochastic nature of the EC model produces a rugged optimization landscape, which prevents us from fitting the parameters $\eta$ and $\gamma$ using conventional optimization algorithms. In order to circumvent this problem, we instead fit the generative model to instances of the EC model, since the generative model has a smooth optimization landscape.

Overall, the procedure consists of the following steps. For a given pair of $(\eta, \gamma)$, we generate ten instances of the EC model. Then, we fit the generative model to the population of simulated EC models and record the optimal parameters SA- $\lambda$ and TA- $\Delta_{1}$. We repeat these steps over a predetermined range of $\eta$ and $\gamma$ values that generally produce reasonable networks. On 
a high level, this procedure returns the parameters SA- $\lambda$ and TA- $\Delta_{1}$ of the generative model that fit best to the EC model determined by given values of $\eta$ and $\gamma$. This allows us to conduct further analyses to reveal how changes in the EC model parameters affect changes in the corresponding generative model parameters, thereby linking the two models for comparison.

In order to perform this fitting procedure, we need to establish a similarity metric between the multiple instances of the EC model and the single instance of the generative model. We also require an optimization method for fitting parameters that maximize this similarity metric.

To evaluate the similarity between two networks, we use the energy function presented in Ref. [15],

$$
E=\max \left(K S_{k}, K S_{c}, K S_{b}, K S_{e}\right)
$$

where each argument in the max function is the two-sample Kolmogorov-Smirnov test statistic between the networks' degree $(k)$, clustering $(c)$, betweenness centrality $(b)$, and edge length $(e)$ distributions. Note that for consistency with Ref. [15], this energy function measures fitness based on nodal graph metrics, in contrast to the energy function for fitting the generative model to data, which is based on eigenvalues.

To reduce noise and to produce population-level fits, we collectively minimize the average energy between all instances of the EC model and a single instance of the generative model,

$$
E_{\text {avg }}=\frac{1}{n} \sum_{i=1}^{n} \max \left(K S_{k}^{i}, K S_{c}^{i}, K S_{b}^{i}, K S_{e}^{i}\right)
$$

where $n$ is the number of subjects in our dataset, and each argument to the max function is the Kolmogorov-Smirnov statistic between the distributions of $i$ th instance of the generative graph model and the generative model for the corresponding graph metric. Instances of the EC model differ only in the initial random seed. We used differential evolution to determine the optimal model parameters.

\subsection{Results}

One apparent similarity between the construction of the EC model and the homogeneous generative model is that each model includes a parameter that controls connection probability with respect to distance between nodes: the distance parameter $\eta$ in the EC model and the SA- $\lambda$ parameter in the homogeneous generative model. As $\eta \rightarrow 0^{-}$, distance plays a smaller role in determining connection probability, so long- and short-range connections are more equally likely to be chosen. As SA- $\lambda \rightarrow 00^{+}$, the spatial autocorrelation approaches zero for all non-zero distances, so distance has a diminishing effect on the correlation. Therefore, we expect the EC distance parameter $\eta$ to be negatively correlated with SA- $\lambda$. To test this empirically, we fix the EC clustering parameter at several different values and vary the EC distance parameter, fitting the homogeneous generative model to each of the EC model's simulated networks. We find a strong negative relationship between the EC distance parameter $\eta$ and SA- $\lambda$ (Figure S8a). This confirms the similarities expected between the two spatial parameters.

The ability of both models to capture network topology, combined with the strong association of SA- $\lambda$ and EC distance parameter, suggests that the second parameters of these two models may also be related. Specifically, we test whether the clustering parameter $\gamma$ in the EC model and TA- $\Delta_{1}$ in the homogeneous generative model are associated as well. After applying 
Shinn et al.

the same procedure of simulating from the EC model and fitting with the homogeneous generative model, we found a tight relationship between TA- $\Delta_{1}$ and the EC clustering parameter (Figure S8b). Indeed, varying these parameters in the two models results in similar changes in graph metrics (Figure S9). By contrast, the TA- $\Delta_{1}$ parameter is not strongly associated with the EC distance parameter (Figure S8c), and the SA- $\lambda$ parameter is not strongly associated with the EC clustering parameter (Figure S8d). Thus, increases in SA breadth reduce the relative probability of nearby connections, and increases in TA increase the probability of connections occurring preferentially within clusters. This confirms our intuition of how SA and TA influence graph topology.

\section{References}

[86] Bullmore, E. et al. Colored noise and computational inference in neurophysiological (fMRI) time series analysis: Resampling methods in time and wavelet domains. Human Brain Mapping 12, 61-78 (2001).

[87] Wagenmakers, E.-J., Farrell, S. \& Ratcliff, R. Estimation and interpretation of 1/f $\alpha$ noise in human cognition. Psychonomic bulletin \& review 11, 579-615 (2004).

[88] Maxim, V. et al. Fractional Gaussian noise, functional MRI and Alzheimer's disease. NeuroImage 25, 141-158 (2005).

[89] Scheffer, M. et al. Early-warning signals for critical transitions. Nature 461, 53-59 (2009).

[90] Lu, Z. Analysis of stationary and non-stationary long memory processes : estimation, applications and forecast. Theses, École normale supérieure de Cachan - ENS Cachan (2009).

[91] Hassani, H., Leonenko, N. \& Patterson, K. The sample autocorrelation function and the detection of long-memory processes. Physica A: Statistical Mechanics and its Applications 391, 6367-6379 (2012).

[92] Achard, S., Bassett, D. S., Meyer-Lindenberg, A. \& Bullmore, E. Fractal connectivity of long-memory networks. Physical Review E 77, 036104 (2008).

[93] Sela, R. J. \& Hurvich, C. M. Computationally efficient methods for two multivariate fractionally integrated models. Journal of Time Series Analysis 30, 631-651 (2009).

[94] Achard, S. \& Gannaz, I. Multivariate wavelet whittle estimation in long-range dependence. Journal of Time Series Analysis 37, 476-512 (2015).

[95] Timmer, J. \& König, M. On Generating Power Law Noise. A\&a 300, 707-710 (1995).

[96] Yule, G. U. Why do we sometimes get nonsense-correlations between time-series?-a study in sampling and the nature of time-series. Journal of the Royal Statistical Society 89, 1 (1926).

[97] Arbabshirani, M. R. et al. Impact of autocorrelation on functional connectivity. NeuroImage 102, 294-308 (2014).

[98] Schaworonkow, N., Blythe, D. A. J., Kegeles, J., Curio, G. \& Nikulin, V. V. Power-law dynamics in neuronal and behavioral data introduce spurious correlations. Human Brain Mapping 36, 2901-2914 (2015).

[99] Afyouni, S., Smith, S. M. \& Nichols, T. E. Effective degrees of freedom of the Pearson's correlation coefficient under autocorrelation. NeuroImage 199, 609-625 (2019).

[100] Watts, D. J. \& Strogatz, S. H. Collective dynamics of 'small-world' networks. Nature 393, 440-442 (1998).

[101] Barabási, A.-L. \& Albert, R. Emergence of scaling in random networks. Science 286, 509-512 (1999).

[102] Albert, R. \& Barabási, A.-L. Statistical mechanics of complex networks. Reviews of Modern Physics 74, 47-97 (2002).

[103] Fornito, A., Zalesky, A. \& Bullmore, E. Fundamentals of Brain Network Analysis (Academic Press, 2016). 
[104] Bassett, D. S. \& Sporns, O. Network neuroscience. Nature Neuroscience 20, 353-364 (2017).

[105] Betzel, R. F. et al. The modular organization of human anatomical brain networks: Accounting for the cost of wiring. Network Neuroscience 1, 42-68 (2017).

[106] Vértes, P. E. et al. Simple models of human brain functional networks. Proceedings of the National Academy of Sciences 109, 5868-5873 (2012).

[107] Morgan, S. E., Achard, S., Termenon, M., Bullmore, E. T. \& Vértes, P. E. Low-dimensional morphospace of topological motifs in human fMRI brain networks. Network Neuroscience 2, 285-302 (2018).

[108] Hinne, M., Heskes, T., Beckmann, C. F. \& van Gerven, M. A. Bayesian inference of structural brain networks. NeuroImage 66, 543-552 (2013).

[109] Klimm, F., Bassett, D. S., Carlson, J. M. \& Mucha, P. J. Resolving structural variability in network models and the brain. PLoS Computational Biology 10, e1003491 (2014).

[110] Betzel, R. F. et al. Generative models of the human connectome. NeuroImage 124, 1054-1064 (2016).

[111] Tang, E. et al. Developmental increases in white matter network controllability support a growing diversity of brain dynamics. Nature Communications 8 (2017).

[112] Akarca, D., Vértes, P. E., Bullmore, E. T. \& and, D. E. A. A generative network model of neurodevelopment. Biorxiv (2020).

[113] Bullmore, E. \& Sporns, O. The economy of brain network organization. Nature Reviews Neuroscience 13, 336-349 (2012).

[114] Kruskal, J. B. On the shortest spanning subtree of a graph and the traveling salesman problem. Proceedings of the American Mathematical Society 7, 48-48 (1956). 
bioRxiv preprint doi: https://doi.org/10.1101/2021.06.01.446561; this version posted June 1, 2021. The copyright holder for this preprint (which was not certified by peer review) is the author/funder. All rights reserved. No reuse allowed without permission.

Shinn et al.

Table S1 | Graph metric fits for all models. For each model, in each dataset, the single subject fits are shown for assortativity, clustering, local efficiency, global efficiency, modularity, and transitivity (top) and SA- $\lambda$, SA- $\infty$, TA- $\Delta_{1}$, mean-FC, var-FC, and kurt-FC (bottom). Fits are quantified using Lin's concordance, Spearman correlation $r_{s}$, and coefficient of determination $R^{2}$.

\begin{tabular}{|c|c|c|c|c|c|c|c|c|c|c|c|c|c|c|c|c|c|c|c|}
\hline \multirow[b]{2}{*}{ Dataset } & \multirow[b]{2}{*}{ Model } & \multicolumn{2}{|c|}{ Assortativity } & \multirow[b]{2}{*}{$R^{2}$} & \multicolumn{2}{|c|}{ Clustering coef. } & \multirow[b]{2}{*}{$R^{2}$} & \multicolumn{2}{|c|}{ Local efficiency } & \multirow[b]{2}{*}{$R^{2}$} & Globa & iciency & & Modula & & & Transit & & \\
\hline & & Lin & $r_{s}$ & & Lin & $r_{s}$ & & Lin & $r_{s}$ & & Lin & $r_{s}$ & $R^{2}$ & Lin & $r_{s}$ & $R^{2}$ & Lin & $r_{s}$ & $R^{2}$ \\
\hline hop & Data (retest) & 0.39 & 0.39 & -0.22 & 0.37 & 0.39 & -0.26 & 0.42 & 0.44 & -0.17 & 0.4 & 0.38 & -0.22 & 0.45 & 0.47 & -0.12 & 0.53 & 0.52 & 0.04 \\
\hline & Generative model & 0.43 & 0.52 & -0.4 & 0.63 & 0.63 & 0.33 & 0.74 & 0.79 & 0.54 & 0.2 & 0.32 & -0.77 & 0.6 & 0.7 & 0.38 & 0.45 & 0.6 & -0.66 \\
\hline & SA only & 0.02 & 0.17 & -1.35 & 0.0 & 0.0 & -5.24 & 0.0 & -0.05 & -9.36 & 0.01 & 0.2 & -2.19 & 0.0 & 0.23 & -5.7 & 0.0 & 0.51 & -30.29 \\
\hline & TA only & $\begin{array}{l}0.01 \\
-0.01\end{array}$ & -0.13 & $\begin{array}{r}-1.05 \\
-3.02\end{array}$ & -0.0 & 0.01 & $\begin{array}{r}-89.13 \\
-89.13\end{array}$ & -0.01 & -0.06 & $\begin{array}{l}-3.00 \\
-3.06 \\
-10\end{array}$ & 0.0 & 0.23 & -30.6 & -0.01 & $\begin{array}{l}0.20 \\
-0.16\end{array}$ & $\begin{array}{l}-3.1 \\
-3.78\end{array}$ & 0.0 & 0.17 & -12.59 \\
\hline & Noiseless & 0.34 & 0.41 & 0.13 & -0.01 & -0.17 & -98.83 & -0.07 & -0.16 & -4.5 & 0.01 & 0.46 & -33.47 & -0.1 & -0.3 & -3.39 & 0.02 & 0.37 & -12.54 \\
\hline & Phase randomization & -0.02 & -0.13 & -1.99 & -0.01 & -0.39 & -128.6 & -0.06 & -0.42 & -7.96 & 0.01 & 0.31 & -37.51 & -0.06 & -0.43 & -5.11 & -0.0 & -0.06 & -14.67 \\
\hline & Zalesky matching & 0.16 & 0.37 & -0.15 & -0.02 & -0.48 & -75.8 & -0.23 & -0.56 & -2.71 & 0.02 & 0.37 & -28.21 & -0.02 & -0.27 & -5.23 & -0.0 & -0.04 & -10.98 \\
\hline & Edge reshuffle & 0.0 & -0.06 & -6.79 & -0.05 & -0.64 & -77.28 & -0.1 & -0.7 & -10.35 & 0.03 & 0.44 & -14.24 & 0.02 & 0.91 & -10.81 & 0.01 & 0.26 & -12.25 \\
\hline hepgsr & Data (retest) & 0.43 & 0.43 & -0.15 & 0.39 & 0.39 & -0.23 & 0.44 & 0.45 & -0.13 & 0.42 & 0.41 & -0.16 & 0.42 & 0.43 & -0.18 & 0.53 & 0.53 & 0.06 \\
\hline 10pgsi & Generative model & 0.34 & 0.46 & -0.57 & 0.37 & 0.37 & -0.27 & 0.52 & 0.68 & -0.34 & 0.16 & 0.28 & -1.2 & 0.36 & 0.49 & -0.36 & 0.23 & 0.37 & -1.95 \\
\hline & SA only & 0.01 & 0.15 & -0.08 & 0.0 & -0.04 & -5.33 & -0.0 & -0.09 & -13.2 & 0.01 & 0.14 & -3.09 & 0.0 & 0.1 & -5.12 & 0.0 & 0.23 & -25.09 \\
\hline & TA only & -0.02 & -0.23 & -4.48 & 0.0 & 0.14 & -173.28 & 0.01 & 0.19 & -14.99 & 0.0 & 0.32 & -45.63 & 0.0 & 0.05 & -14.31 & 0.0 & 0.32 & -19.98 \\
\hline & Noiseless & 0.28 & 0.43 & -0.18 & 0.0 & 0.01 & -181.85 & 0.01 & 0.06 & -16.87 & 0.01 & 0.32 & -50.21 & -0.01 & -0.06 & -11.23 & 0.01 & 0.42 & -20.14 \\
\hline & Phase randomization & -0.03 & -0.28 & -2.85 & -0.0 & -0.08 & -257.15 & -0.01 & -0.11 & -33.41 & 0.0 & 0.23 & -57.72 & -0.01 & -0.21 & -17.76 & 0.0 & 0.13 & -23.19 \\
\hline & Zalesky matching & 0.12 & 0.28 & -0.45 & -0.0 & -0.25 & -181.39 & -0.03 & -0.28 & -14.85 & 0.0 & 0.23 & -48.29 & 0.0 & 0.18 & -16.84 & 0.0 & 0.06 & -19.88 \\
\hline & Edge reshuffle & -0.02 & -0.32 & -6.93 & -0.01 & -0.4 & -193.51 & -0.03 & -0.6 & -41.27 & 0.01 & 0.34 & -25.04 & 0.01 & 0.76 & -28.89 & -0.0 & -0.05 & -19.2 \\
\hline trt & Data (retest) & 0.13 & 0.15 & -0.94 & 0.31 & 0.28 & -0.42 & 0.41 & 0.4 & -0.29 & 0.21 & 0.21 & -0.68 & 0.36 & 0.36 & -0.33 & 0.24 & 0.24 & -0.6 \\
\hline & Generative model & -0.12 & -0.17 & -1.65 & 0.16 & 0.55 & -4.14 & 0.2 & 0.5 & -4.13 & 0.22 & 0.55 & -1.47 & 0.02 & 0.24 & -32.27 & 0.1 & 0.31 & -3.24 \\
\hline & SA only & 0.01 & 0.43 & -3.84 & 0.02 & 0.75 & -5.49 & 0.0 & 0.47 & -19.72 & 0.02 & 0.8 & -6.5 & -0.0 & 0.03 & -0.63 & 0.01 & 0.82 & -11.45 \\
\hline & TA only & 0.0 & 0.05 & -4.24 & 0.0 & 0.02 & -29.49 & -0.0 & -0.01 & -11.93 & -0.0 & -0.05 & -14.58 & 0.0 & 0.11 & -23.11 & -0.0 & -0.02 & -12.26 \\
\hline & Noiseless & 0.02 & 0.04 & -1.42 & 0.01 & 0.34 & -29.85 & 0.03 & 0.5 & -16.04 & 0.01 & 0.23 & -15.13 & 0.01 & 0.17 & -28.83 & 0.01 & 0.32 & -12.63 \\
\hline & Phase randomization & -0.0 & -0.02 & -6.2 & 0.0 & 0.32 & -75.2 & 0.0 & 0.27 & -72.3 & 0.0 & 0.3 & -35.41 & 0.0 & 0.22 & -68.85 & 0.0 & 0.28 & -24.73 \\
\hline & Zalesky matching & 0.06 & 0.1 & -2.19 & 0.01 & 0.65 & -57.6 & 0.02 & 0.58 & -36.39 & 0.01 & 0.63 & -28.49 & 0.01 & 0.4 & -55.84 & 0.01 & 0.68 & -21.15 \\
\hline & Edge reshuffle & -0.01 & -0.27 & -15.41 & 0.01 & 0.47 & -103.56 & -0.0 & -0.11 & -196.35 & 0.03 & 0.71 & -27.9 & -0.0 & -0.13 & -126.97 & 0.01 & 0.43 & -24.62 \\
\hline camcan & Generative model & 0.16 & 0.39 & -5.84 & 0.08 & 0.11 & -0.5 & 0.11 & 0.13 & -0.56 & 0.16 & 0.25 & -2.08 & 0.1 & 0.16 & -1.08 & 0.21 & 0.4 & -3.24 \\
\hline & SA only & 0.03 & 0.41 & -1.92 & 0.0 & 0.13 & -4.37 & 0.0 & 0.1 & -8.3 & 0.03 & 0.24 & -0.41 & -0.0 & 0.01 & -4.09 & 0.01 & 0.4 & -8.48 \\
\hline & TA only & -0.0 & -0.04 & -6.79 & 0.01 & 0.06 & -2.27 & 0.01 & 0.05 & -2.36 & -0.0 & $\begin{array}{l}-0.04 \\
-0.04\end{array}$ & -0.42 & -0.0 & -0.04 & -3.31 & -0.02 & -0.07 & -0.92 \\
\hline & Noiseless & -0.01 & -0.04 & -1.28 & 0.2 & 0.2 & -0.42 & 0.14 & 0.25 & -0.95 & -0.02 & -0.05 & -1.71 & 0.16 & 0.28 & -1.02 & 0.14 & 0.09 & -0.72 \\
\hline & Phase randomization & -0.02 & -0.03 & -5.63 & -0.23 & $\begin{array}{l}-0.32 \\
-0.32 y\end{array}$ & -4.66 & -0.28 & -0.32 & -2.03 & 0.04 & 0.14 & -6.02 & -0.2 & -0.32 & -1.95 & -0.3 & -0.35 & -1.52 \\
\hline & Zalesky matching & 0.03 & 0.04 & -1.48 & -0.24 & -0.28 & -1.86 & -0.26 & -0.31 & -1.13 & 0.1 & 0.31 & -4.12 & -0.23 & -0.28 & -1.19 & -0.15 & -0.18 & -2.69 \\
\hline & Edge reshuffle & -0.0 & -0.02 & -17.84 & -0.08 & -0.63 & -22.34 & -0.15 & -0.7 & -8.35 & 0.01 & 0.45 & -17.11 & 0.03 & 0.77 & -8.02 & 0.01 & 0.3 & -12.15 \\
\hline & & $\mathrm{SA}-\lambda$ & & & $\mathrm{SA}-\infty$ & & & Mean & & & Mean- & & & Var-FC & & & Kurt-F & & \\
\hline Dataset & Model & Lin & $r_{s}$ & $R^{2}$ & Lin & $r_{s}$ & $R^{2}$ & Lin & $r_{s}$ & $R^{2}$ & Lin & $r_{s}$ & $R^{2}$ & Lin & $r_{s}$ & $R^{2}$ & Lin & $r_{s}$ & $R^{2}$ \\
\hline hсp & Data (retest) & 0.68 & 0.69 & 0.36 & 0.6 & 0.63 & 0.18 & 0.75 & 0.75 & 0.5 & 0.61 & 0.65 & 0.21 & 0.59 & 0.6 & 0.19 & 0.59 & 0.6 & 0.17 \\
\hline & Generative model & 0.39 & 0.82 & -5.67 & 0.84 & 0.94 & 0.53 & 0.99 & 1.0 & 0.97 & 0.85 & 0.97 & 0.55 & 0.21 & 0.83 & -1.9 & 0.81 & 0.93 & 0.48 \\
\hline & SA only & 0.03 & 0.72 & -171.82 & 0.69 & 0.81 & 0.17 & -0.0 & -0.27 & -33.2 & 0.89 & 0.97 & 0.75 & 0.07 & 0.26 & -2.48 & 0.12 & 0.57 & -16.55 \\
\hline & TA only & 0.0 & 0.57 & -14.43 & 0.0 & 0.52 & -3.24 & 1.0 & 1.0 & 1.0 & 0.0 & 0.39 & -3.8 & 0.03 & 0.79 & -7.64 & 0.41 & 0.78 & 0.35 \\
\hline & Noiseless & & 1.0 & & -0.08 & -0.47 & -3.1 & 1.0 & 1.0 & 1.0 & -0.06 & -0.17 & -3.24 & 0.03 & 0.84 & -8.78 & -0.01 & $\begin{array}{r}-0.07 \\
-0.07\end{array}$ & -43.16 \\
\hline & Phase randomization & 0.0 & -0.01 & -14.54 & -0.0 & -0.02 & -3.28 & 1.0 & 1.0 & 1.0 & -0.0 & -0.03 & -3.8 & 0.02 & 0.78 & -9.15 & 0.09 & 0.25 & 0.01 \\
\hline & Zalesky matching & 0.0 & 0.03 & -14.42 & 0.98 & 0.99 & 0.95 & & & & 1.0 & & 1.0 & & 0.99 & 0.98 & -0.01 & -0.16 & -1.16 \\
\hline hcpgsr & Data (retest) & 0.72 & 0.73 & 0.44 & 0.54 & 0.59 & 0.06 & 0.78 & 0.78 & 0.56 & 0.56 & 0.63 & 0.11 & 0.67 & 0.67 & 0.34 & 0.6 & 0.6 & 0.19 \\
\hline mitpysi & Generative model & 0.47 & 0.87 & -4.91 & 0.59 & 0.64 & -0.27 & 0.97 & $\begin{array}{r}.10 \\
1.0\end{array}$ & 0.94 & 0.67 & 0.81 & -0.11 & 0.17 & 0.84 & $\begin{array}{l}-2.63 \\
-2.63 \\
y\end{array}$ & 0.64 & 0.89 & -0.33 \\
\hline & SA only & 0.03 & 0.77 & -166.95 & 0.44 & 0.32 & -0.25 & 0.0 & 0.01 & -34.62 & 0.59 & 0.81 & -0.15 & 0.22 & 0.67 & -1.42 & 0.13 & 0.75 & -22.34 \\
\hline & TA only & 0.0 & 0.14 & -16.36 & 0.0 & 0.18 & -2.22 & & & & -0.0 & -0.18 & -3.23 & 0.02 & 0.8 & -8.68 & 0.28 & 0.74 & -0.05 \\
\hline & Noiseless & & & & -0.18 & -0.25 & -1.39 & 1.0 & 1.0 & 1.0 & -0.13 & -0.23 & -1.64 & 0.02 & 0.84 & -9.75 & -0.02 & -0.28 & -67.02 \\
\hline & Phase randomization & -0.0 & -0.0 & -16.4 & -0.0 & -0.03 & -2.28 & 1.0 & 1.0 & 1.0 & 0.0 & -0.0 & -3.24 & 0.01 & 0.75 & -10.32 & 0.04 & 0.23 & -0.88 \\
\hline & Zalesky matching & 0.0 & 0.04 & -16.29 & 001 & & 0.86 & & & & & & & & 1.0 & & 0.01 & 0.41 & -3.61 \\
\hline trt & Data (retest) & 0.54 & 0.63 & 0.02 & 0.55 & 0.54 & 0.05 & 0.62 & 0.7 & 0.21 & 0.35 & 0.37 & -0.4 & 0.33 & 0.38 & -0.45 & 0.38 & 0.44 & -0.4 \\
\hline & Generative model & 0.18 & 0.49 & -11.93 & 0.02 & 0.13 & -33.06 & 0.96 & 0.99 & 0.92 & 0.0 & 0.09 & $<-10^{5}$ & 0.31 & 0.6 & -0.64 & -0.0 & -0.14 & -9.87 \\
\hline & SA only & 0.01 & 0.35 & -120.14 & 0.01 & 0.06 & -0.85 & -0.0 & -0.29 & $<-10^{4}$ & 0.0 & 0.19 & $<-10^{5}$ & 0.03 & 0.9 & -13.43 & 0.06 & 0.35 & -15.38 \\
\hline & TA only & 0.0 & 0.12 & -11.89 & 0.0 & 0.05 & -1.0 & 0.86 & 1.0 & 0.7 & 0.0 & 0.05 & -3.66 & 0.03 & 0.47 & -3.21 & 0.0 & 0.53 & -10.97 \\
\hline & Noiseless & & & & 0.03 & 0.07 & -1.68 & 0.83 & 0.98 & 0.63 & 0.01 & 0.17 & -422.87 & 0.04 & 0.54 & -5.92 & -0.0 & 0.04 & -7.74 \\
\hline & Phase randomization & -0.0 & -0.11 & -14.4 & -0.0 & -0.01 & -1.36 & 0.93 & 0.99 & 0.85 & -0.03 & -0.05 & -2.18 & 0.01 & 0.51 & -21.3 & 0.05 & 0.53 & -4.52 \\
\hline & Zalesky matching & 0.0 & 0.03 & -14.34 & 0.0 & 0.01 & -1.38 & & & & 0.16 & 0.24 & -2.86 & 0.99 & 0.96 & 0.97 & 0.01 & 0.46 & -10.55 \\
\hline camcan & Generative model & -0.02 & 0.14 & -1.18 & 0.66 & 0.83 & -0.08 & 0.37 & 0.9 & -3.66 & 0.77 & 0.98 & 0.03 & 0.45 & 0.63 & -0.23 & 0.21 & 0.65 & -0.2 \\
\hline & SA only & 0.0 & -0.02 & -10.33 & 0.32 & 0.83 & -2.51 & 0.0 & 0.56 & -532.71 & 0.97 & 0.98 & 0.95 & 0.06 & 0.61 & -1.92 & -0.0 & -0.71 & -0.73 \\
\hline & TA only & -0.0 & -0.1 & -1.58 & 0.0 & 0.36 & -9.96 & 0.7 & 1.0 & -0.06 & 0.0 & 0.33 & -22.98 & -0.01 & -0.21 & -4.03 & -0.01 & -0.4 & -1.16 \\
\hline & Noiseless & 0.0 & 0.07 & -1.08 & -0.01 & -0.19 & -4.11 & 0.86 & 0.99 & 0.65 & 0.06 & 0.3 & -4.41 & -0.05 & -0.2 & -0.4 & -0.01 & -0.11 & -0.66 \\
\hline & Phase randomization & 0.0 & -0.02 & -1.57 & -0.0 & -0.01 & -9.95 & 0.95 & 0.98 & 0.88 & 0.0 & -0.02 & -22.92 & 0.03 & -0.0 & -0.67 & -0.07 & -0.5 & -1.0 \\
\hline & Zalesky matching & 0.0 & -0.02 & -1.51 & 0.71 & 0.87 & 0.52 & & & & 1.0 & 1.0 & 1.0 & 0.93 & 0.94 & 0.86 & 0.41 & 0.67 & 0.21 \\
\hline
\end{tabular}


bioRxiv preprint doi: https://doi.org/10.1101/2021.06.01.446561; this version posted June 1, 2021. The copyright holder for this preprint (which was not certified by peer review) is the author/funder. All rights reserved. No reuse allowed without permission.

HCP-GSR

Correlation among graph metrics

a Assort.

Cluster $* *$ Cluster

Local eff. $\star \star \star \star \star$ Local eff.

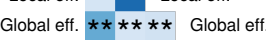

Modularity $* * * * * * *$ Modularity

Transitivity $* * * * \star * * * * *$ Transitivity

Mean-FC $* * \star * * * * \quad * * \star *$ Mean-FC

$\operatorname{Var}-\mathrm{FC} * * * * \quad * * \quad * * * * \operatorname{Var}-\mathrm{FC}$

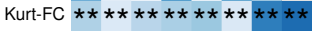

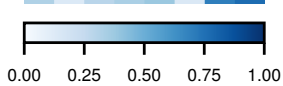

e Reliability of nodal metrics

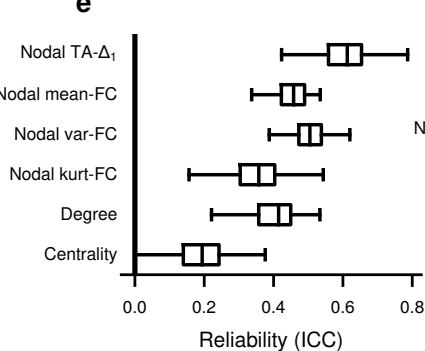

Yale Test-Retest

Correlation among graph metrics

$$
\text { i Assort. }
$$

Assort.
Cluster ** Cluster

Local eff. $\quad * \star$ Local eff.

Global eff. $* * * * \star *$ Global eff.

Modularity $* * \star * \star * * * *$ Modularity

Transitivity $* * * * * * * * * *$ Transitivity

Mean-FC $\quad * * \star * \star * * \star * *$ Mean-FC

$\operatorname{Var}-\mathrm{FC} * * * * * * * * * * * * \star * * \operatorname{Var}-\mathrm{FC}$

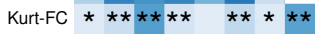

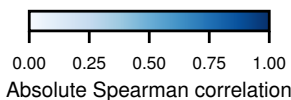

Absolute Spearman correlation

Reliability of nodal metrics

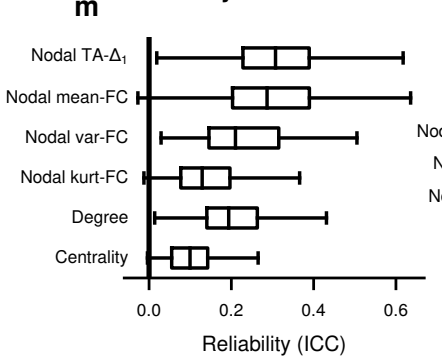

Cam-CAN

Correlation among graph metrics

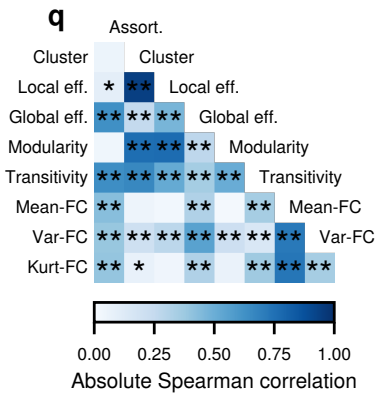

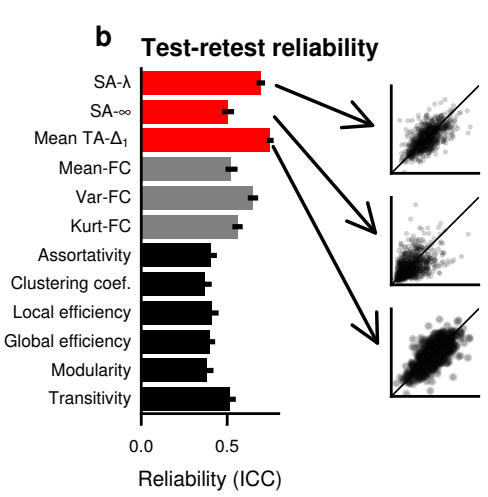

Correlation with graph metrics

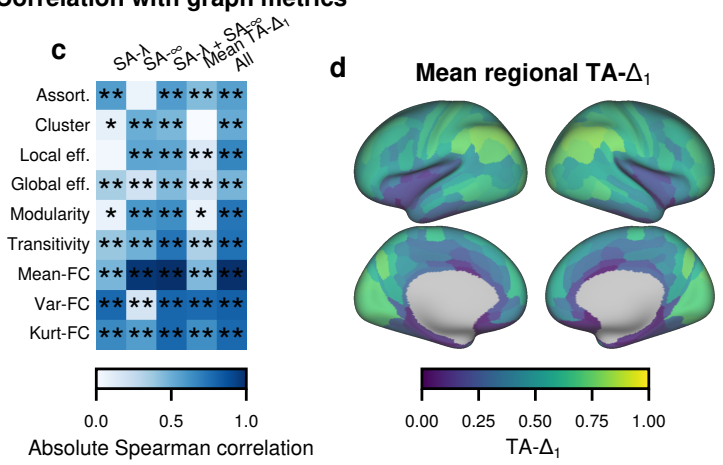

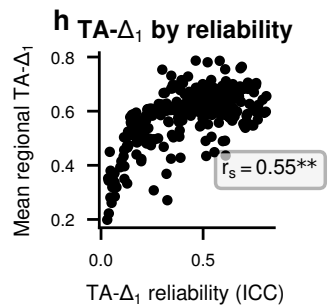

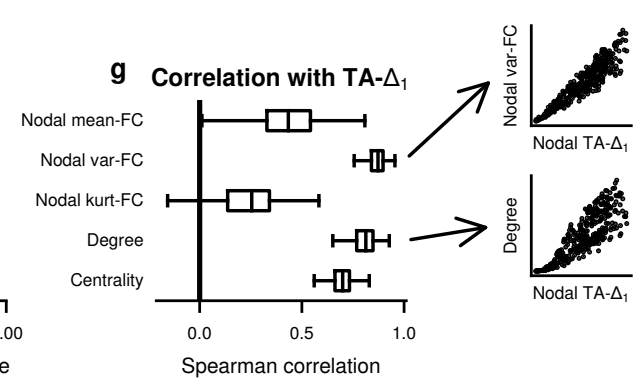

Correlation with graph metrics

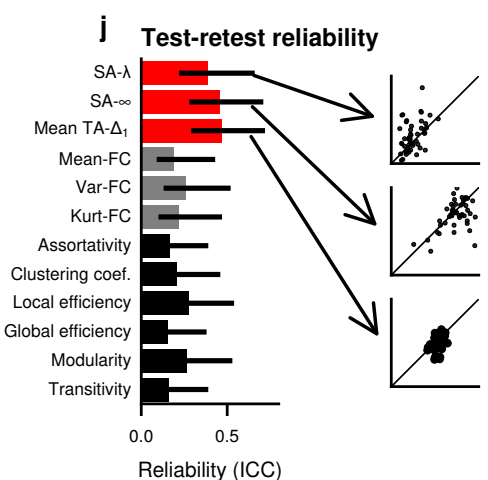

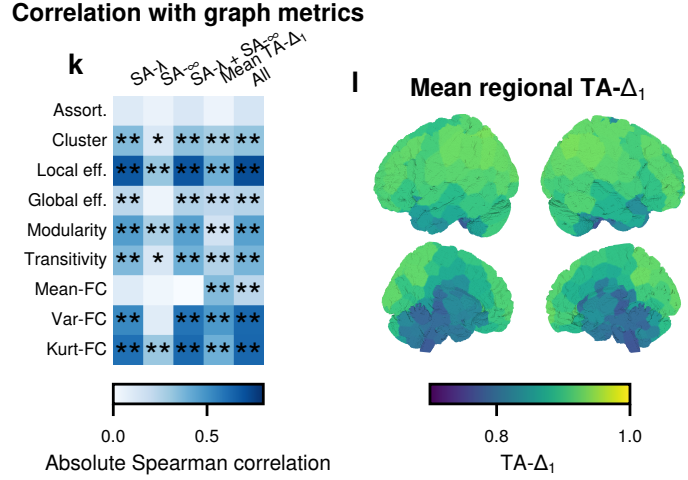
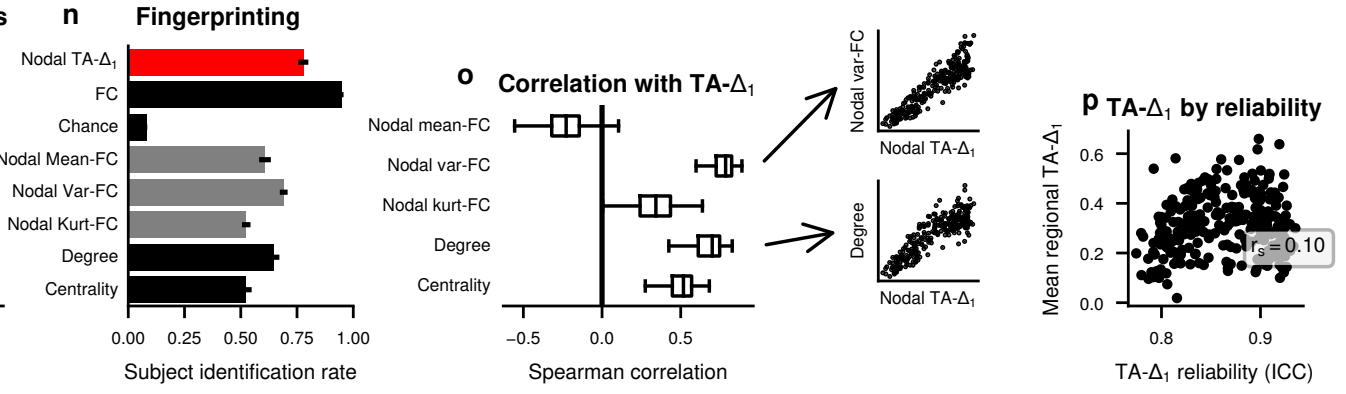

Correlation with graph metrics
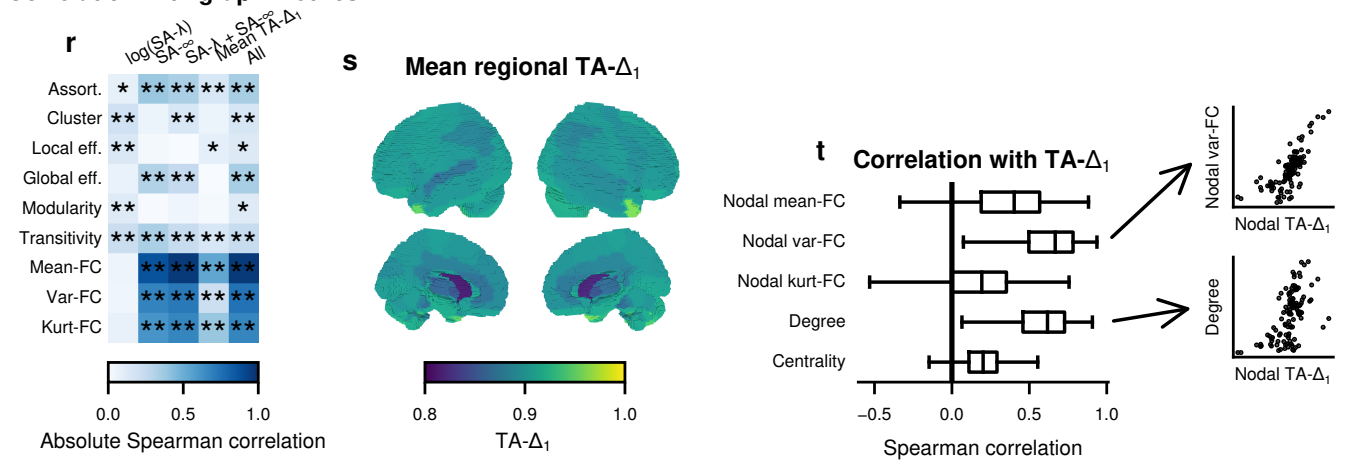

Figure S1 | SA and TA are important features of rs-fMRI. Similar format as Figure 1, but for the HCP-GSR, TRT, and Cam-CAN datasets. 
Shinn et al.

HCP

a Reliability of lag-k autocorrelation

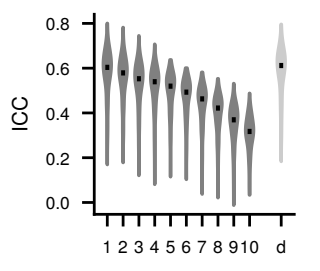

Lag

d Predictive power of lag- 1 for lag-k

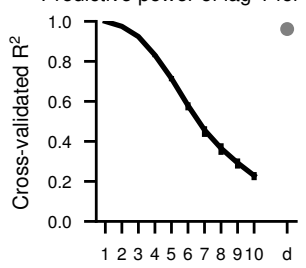

Lag

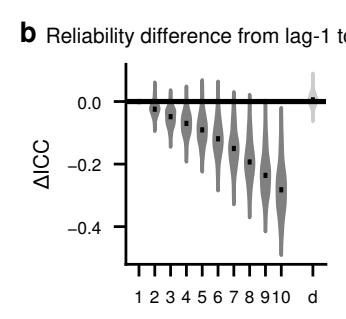

Lag

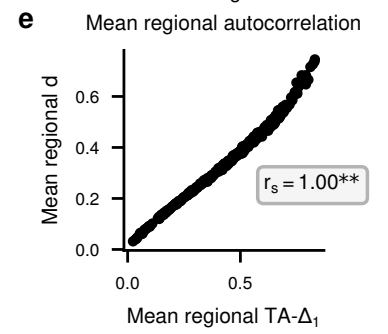

Mean regional TA- $\Delta_{1}$

I Reliability difference from lag-1 to lag-k

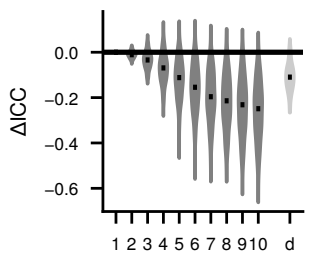

Lag

I Mean regional autocorrelation

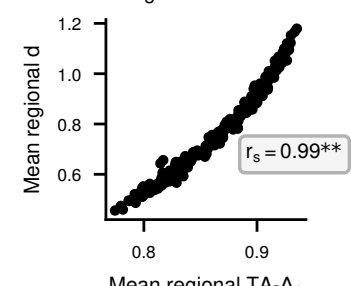

Mean regional TA- $\Delta$
K Predictive power of lag- 1 for lag-k

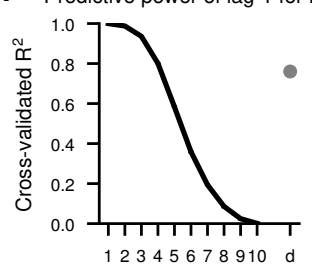

Lag

\section{Cam-CAN}

- Predictive power of lag-1 for lag-k

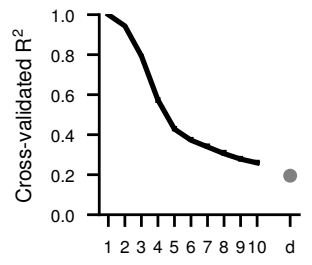

Lag p Mean regional autocorrelation

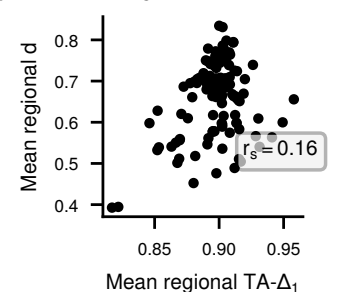

C Reliability of mean lag-k autocorrelation

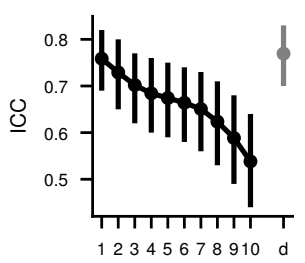

Lag

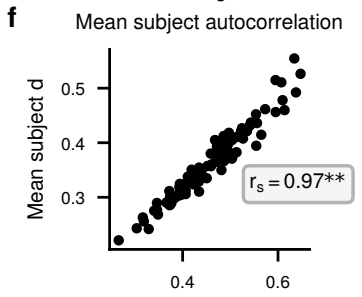

Mean subject TA- $\Delta_{1}$

j Reliability of mean lag-k autocorrelation

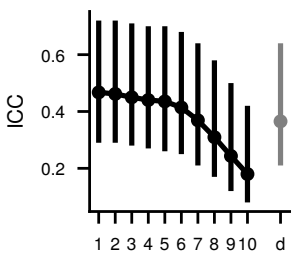

Lag

m Mean subject autocorrelation

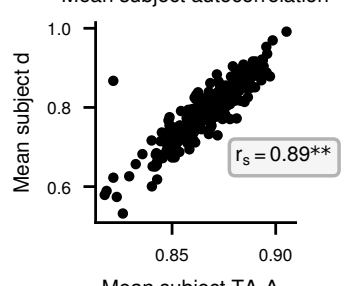

Mean subject TA- $\Delta_{1}$

q Mean subject autocorrelation

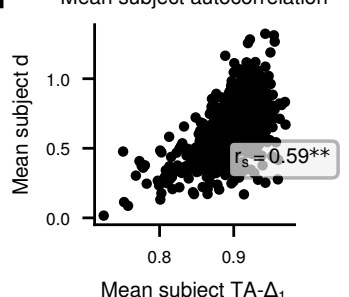

g Subject-region autocorrelation

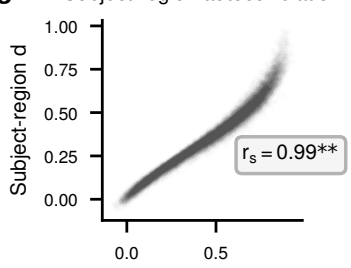

Suject-region TA- $\Delta_{1}$ n Subject-region autocorrelation

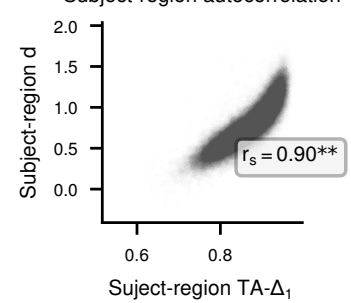

Suject-region TA- $\Delta_{1}$ $\mathbf{r}$

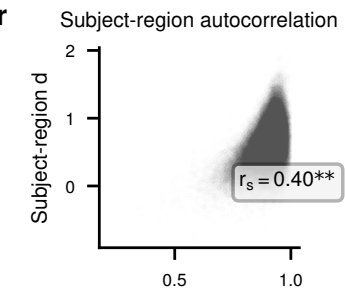

Suject-region TA- $\Delta_{1}$

Figure S2 | TA- $\Delta_{1}$ captures individual variation in long memory dynamics. (a,h) For each brain region, intraclass correlation coefficient (ICC) was computed across runs for the same subject for various lags of the temporal autocorrelation function (ACF). The distribution of reliability across regions is shown in gray, with the median in black. $d$ indicates the fractional integration constant from an ARFIMA $(0, d, 0)$ model fit to the timeseries. $(b, i)$ The distribution of differences in reliability $(\triangle \mathrm{ICC})$ between each lag in the ACF and TA- $\Delta_{1} . d$ indicates the difference in reliability between TA- $\Delta_{1}$ and the fractional integration constant. (c,j) The reliability of the mean TA $-\Delta_{1}$ for each lag, averaged across all regions in each subject, where error bars are $95 \%$ confidence intervals. $d$ indicates reliability of the mean fractional integration constant. $(\mathrm{d}, \mathrm{k}, \mathrm{o})$ A linear model predicted each term of the ACF using TA- $\Delta_{1}$. The model was fit to one subset of the data, and tested on the remaining subsets. Error bars indicate the maximum and minimum $R^{2}$ among the subsets, and are sometimes hidden beneath the curve. $d$ indicates predictions of the fractional integration constant. (e-g,l-n,p-r) Estimates of TA- $\Delta_{1}$ are compared to estimates of $d$. Values were computed for each region, averaging across subjects (e,l,p); for each subject, averaging across regions $(\mathrm{f}, \mathrm{m}, \mathrm{q})$; and for each subject and region, without any averaging $(\mathrm{g}, \mathrm{n}, \mathrm{r}) . r_{s}$ indicates Spearman correlation, where * indicates $p<.05$, and ** indicates $p<.01$. 
bioRxiv preprint doi: https://doi.org/10.1101/2021.06.01.446561; this version posted June 1, 2021. The copyright holder for this preprint (which was not certified by peer review) is the author/funder. All rights reserved. No reuse allowed without permission.

Spatial and temporal autocorrelation

HCP:

a motion on SA- $\lambda$

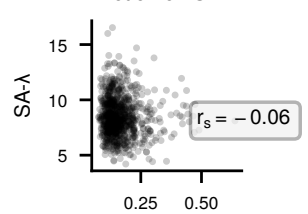

HCP-GSR:

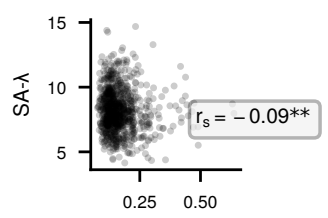

Yale Test-Retest:

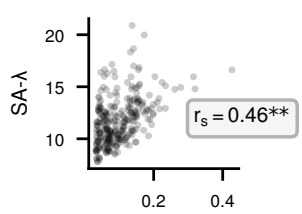

Cam-CAN:

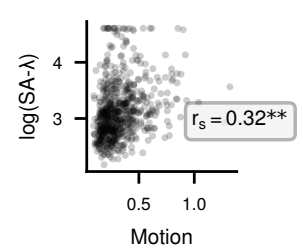

b Effect of head

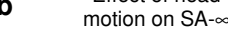
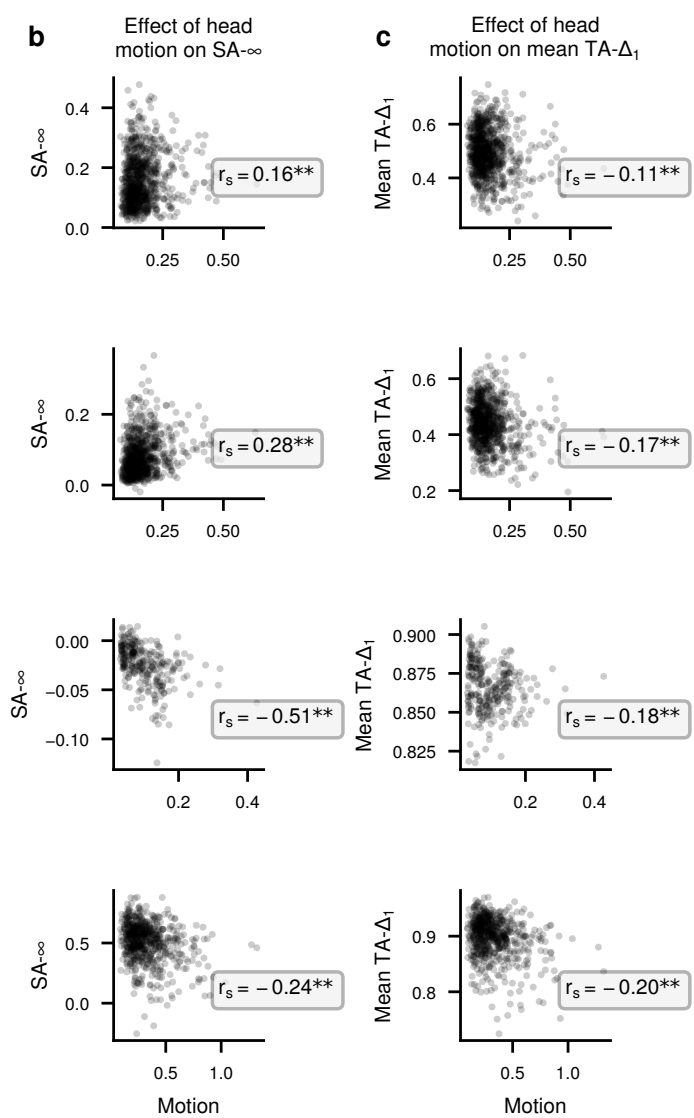
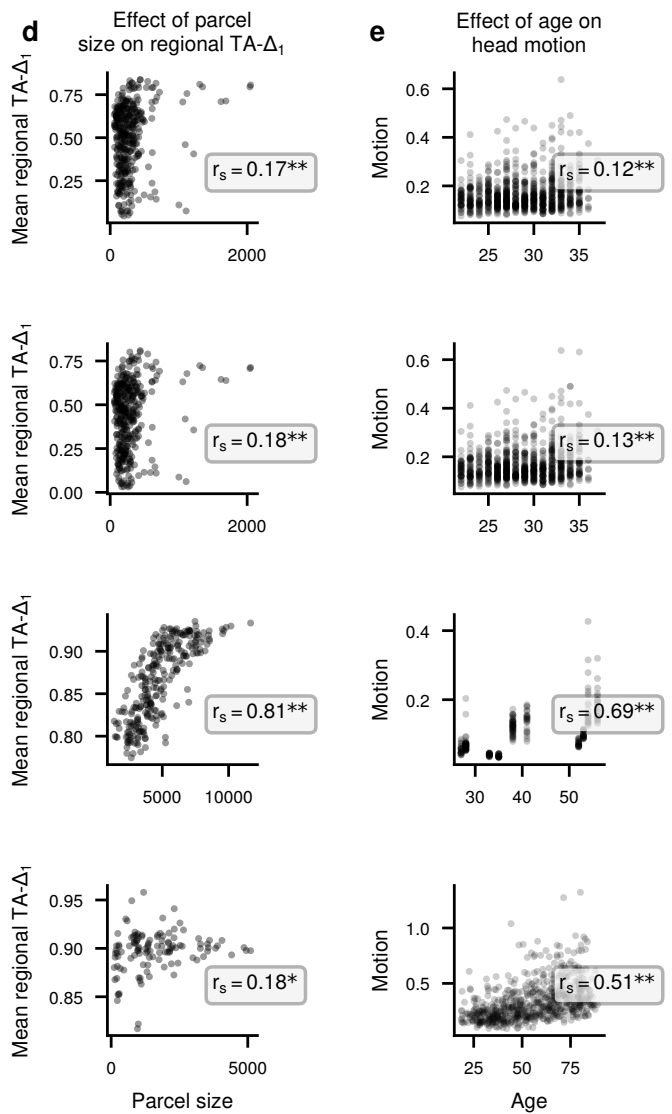

Figure S3 | Correlation of motion and parcel size with spatial and temporal autocorrelation. (a-c) For each dataset, the mean framewise displacement ("motion") is compared to (a) SA- $\lambda$, (b) SA- $\infty$, and (c) mean TA- $\Delta_{1}$, with inset Spearman correlation ( $r_{s}$ ). (d) The average TA- $\Delta_{1}$ for each parcel across all subjects is plotted against the parcel's size, with inset Spearman correlation. Parcel size is measured in surface area for HCP and HCP-GSR, and number of voxels for Yale Test-Retest and Cam-CAN. (e) Subject's mean framewise displacement ("motion") is plotted against the subject's age. * indicates Spearman correlation $p<.05$, and ** indicates $p<.01$. 
Shinn et al.
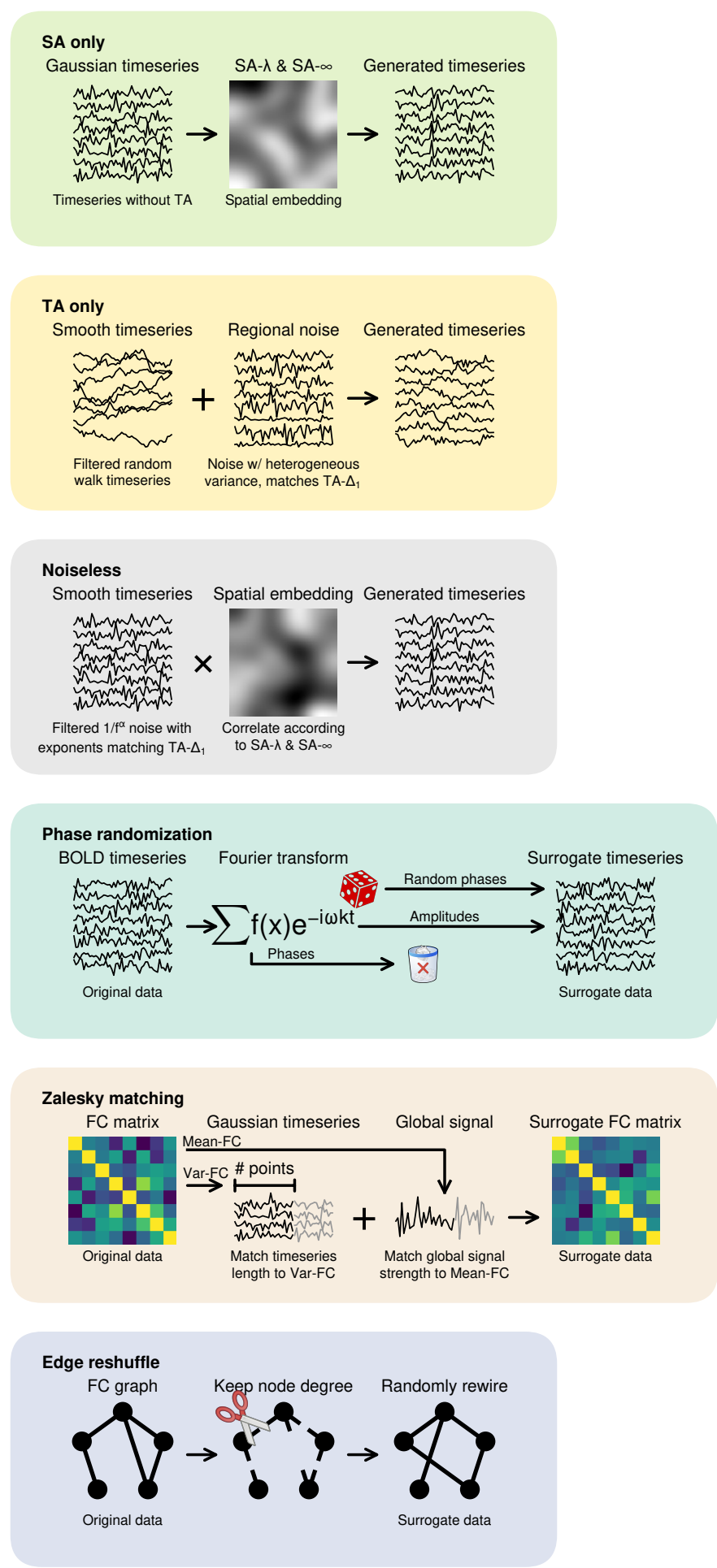

Figure S4 | Schematics of all of the models. All models considered are described in their corresponding schematics. 
bioRxiv preprint doi: https://doi.org/10.1101/2021.06.01.446561; this version posted June 1, 2021. The copyright holder for this preprint (which was not certified by peer review) is the author/funder. All rights reserved. No reuse allowed without permission.
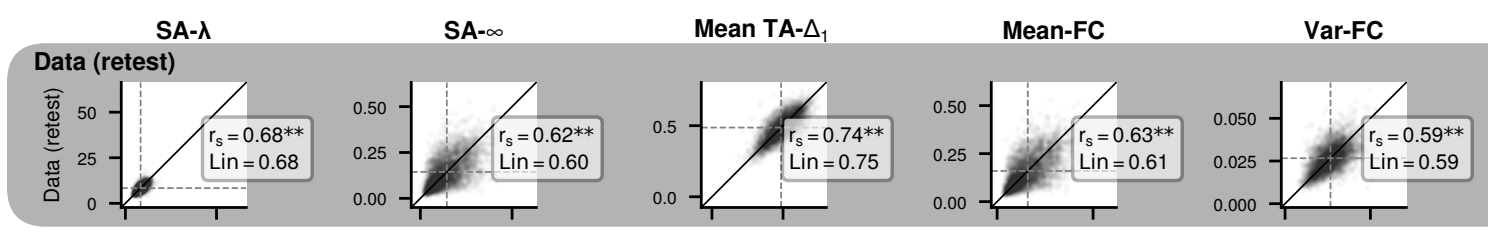

Kurt-FC
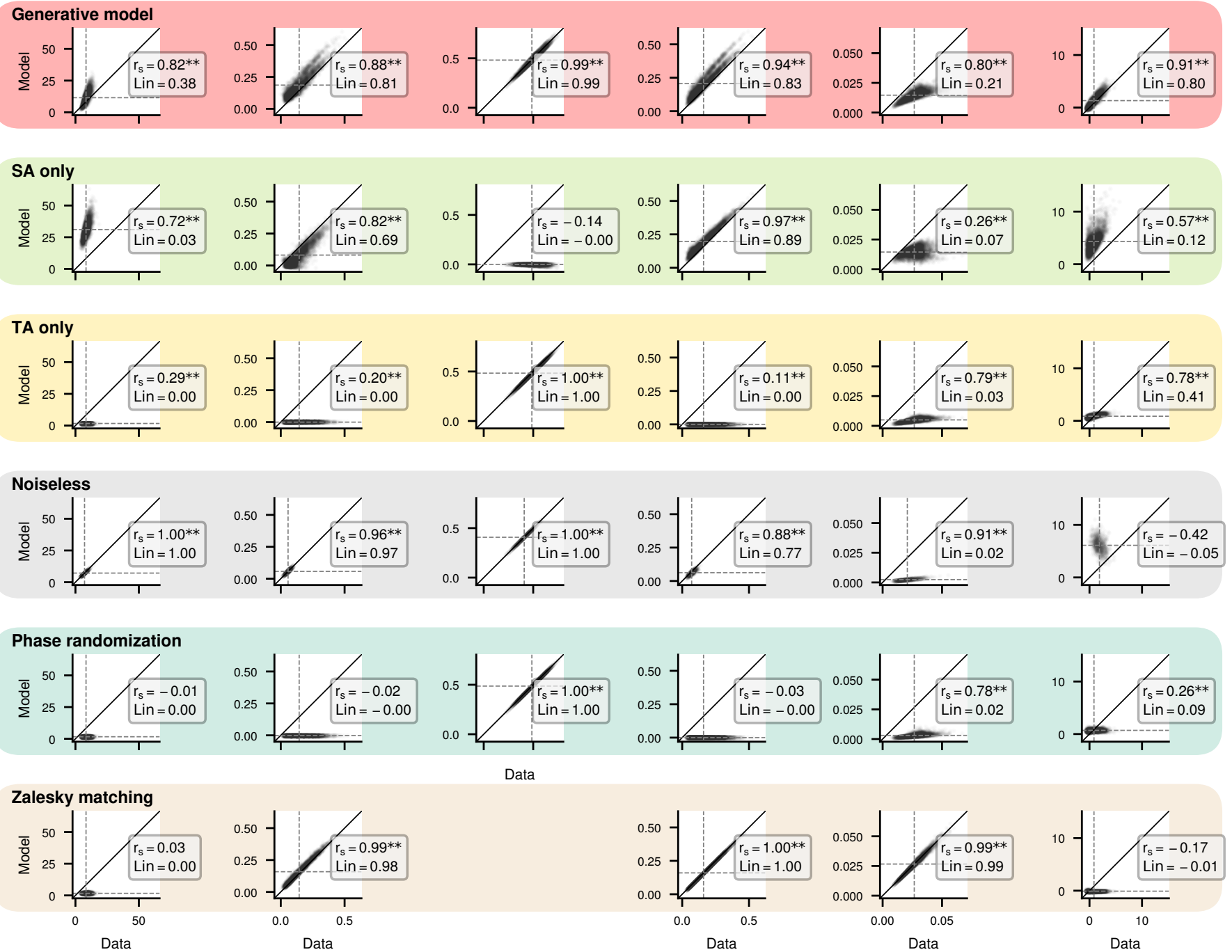

Data

Figure S5 | Correlation of model and data graph metrics for all models in HCP data. For each model, each subject's empirical graph metrics are plotted against model graph metrics for metrics from Figure 2i-p for the HCP dataset. Spearman correlation $\left(r_{s}\right)$ and Lin's concordance (Lin) are inset. * indicates Spearman correlation $p<.05$, and ${ }^{* *}$ indicates $p<.01$. Zalesky matching operates at the level of the FC matrix, and thus, TA- $\Delta_{1}$ could not be computed. Edge reshuffle operates at the level of the graph, preventing any of these measures from being computed. 
Shinn et al.
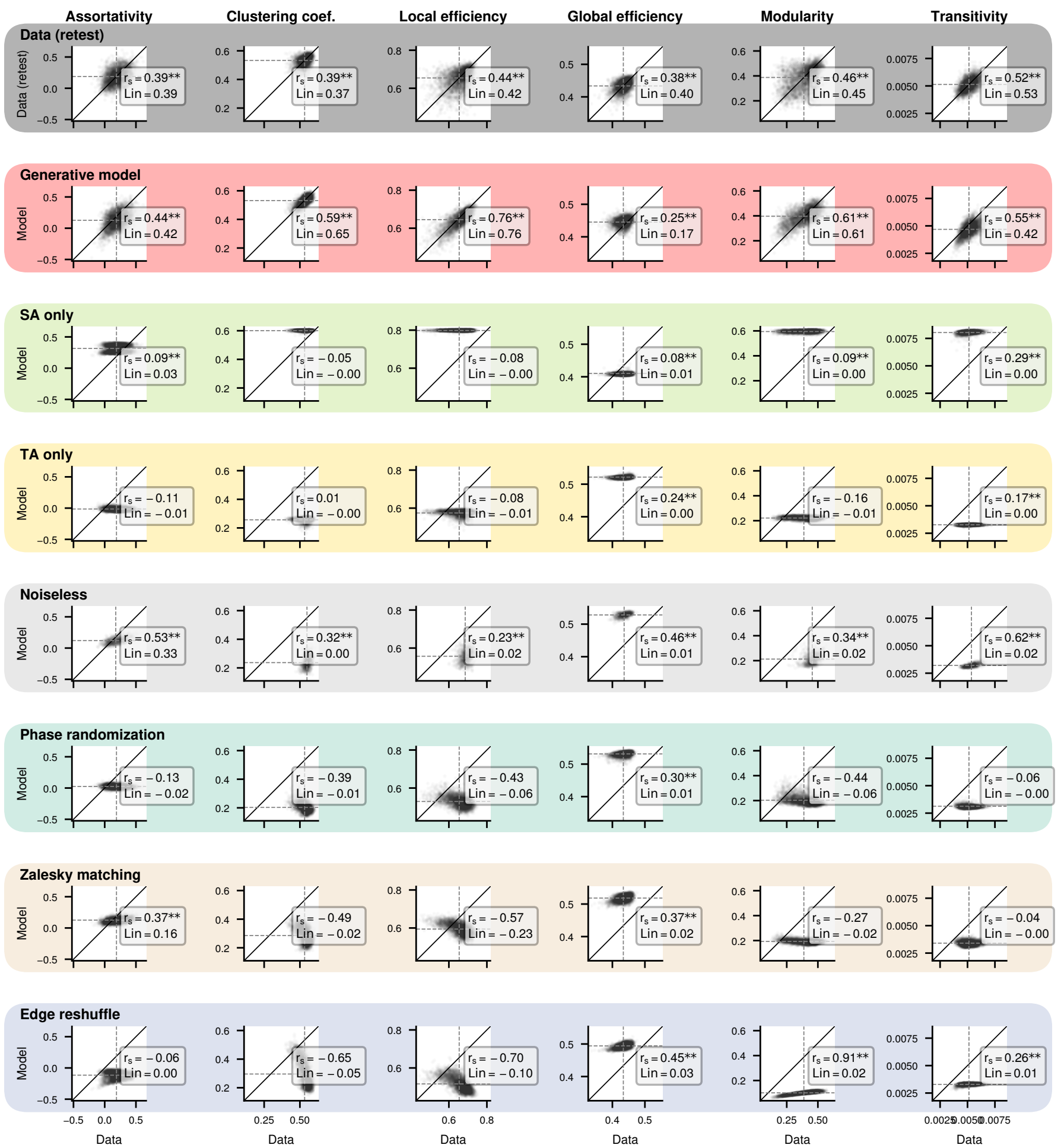

Figure S6 | Correlation of model and data graph metrics for all models in HCP data. For each model, each subject's empirical graph metrics are plotted against model graph metrics for metrics from Figure 2i-p for the HCP dataset. Spearman correlation $\left(r_{s}\right)$ and Lin's concordance (Lin) are inset. * indicates Spearman correlation $p<.05$, and ${ }^{* \star}$ indicates $p<.01$. 
bioRxiv preprint doi: https://doi.org/10.1101/2021.06.01.446561; this version posted June 1, 2021. The copyright holder for this preprint (which was not certified by peer review) is the author/funder. All rights reserved. No reuse allowed without permission.

Spatial and temporal autocorrelation
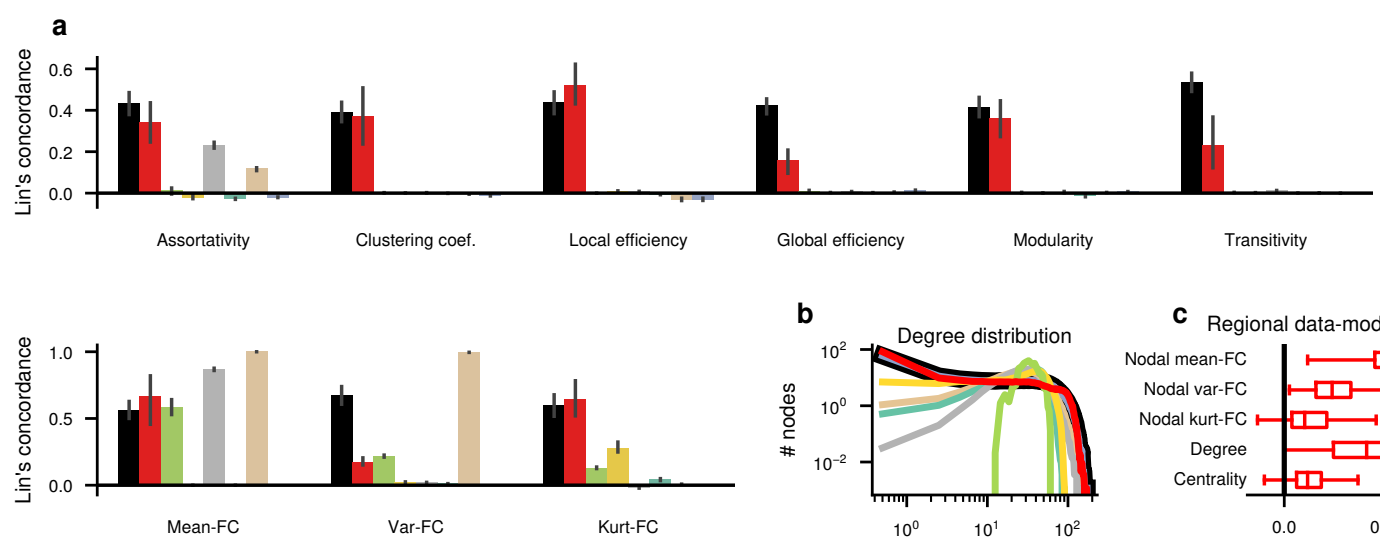

Data (retest) Generative model SA only TA only

Noiseless

Phase randomization Zalesky matching Edge reshuffle
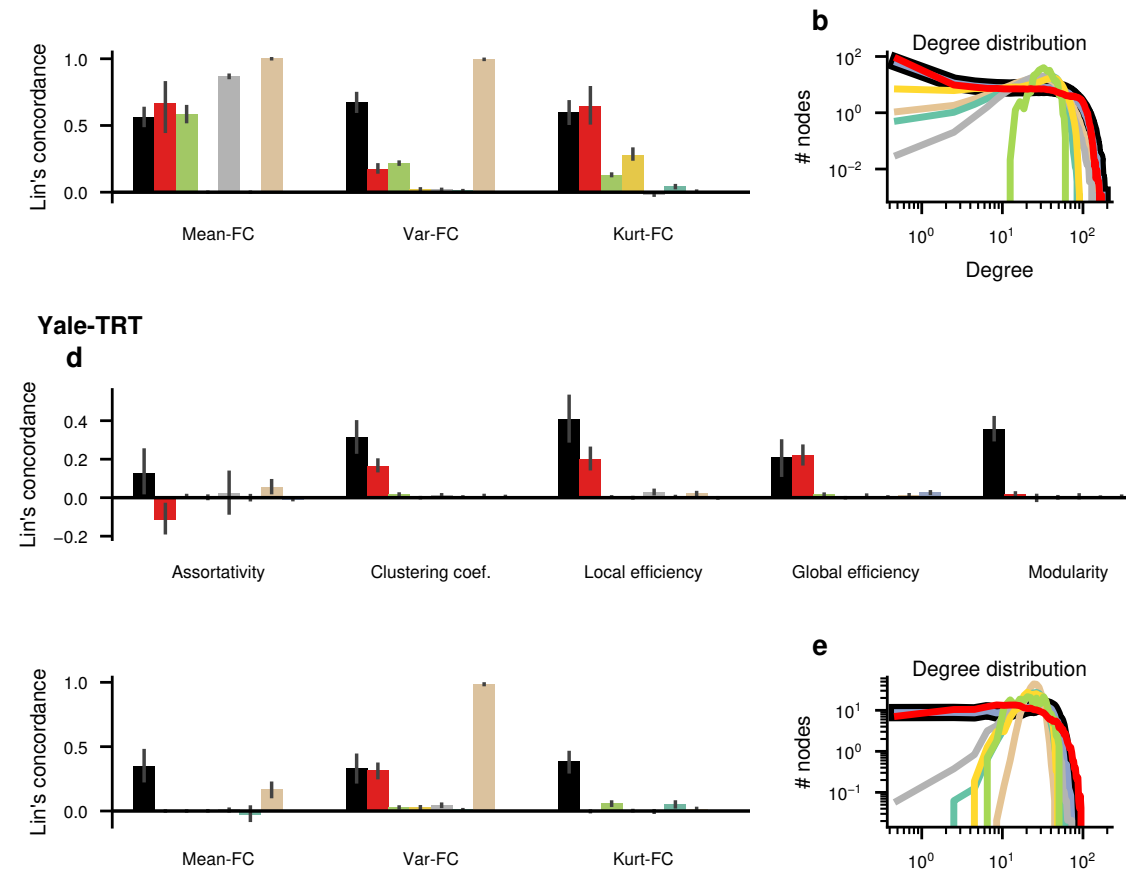

C Regional data-model correlations

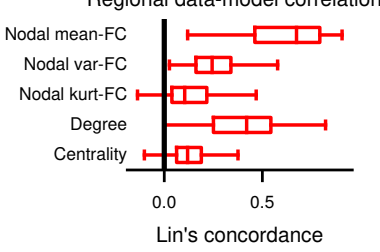

Yale-TRT
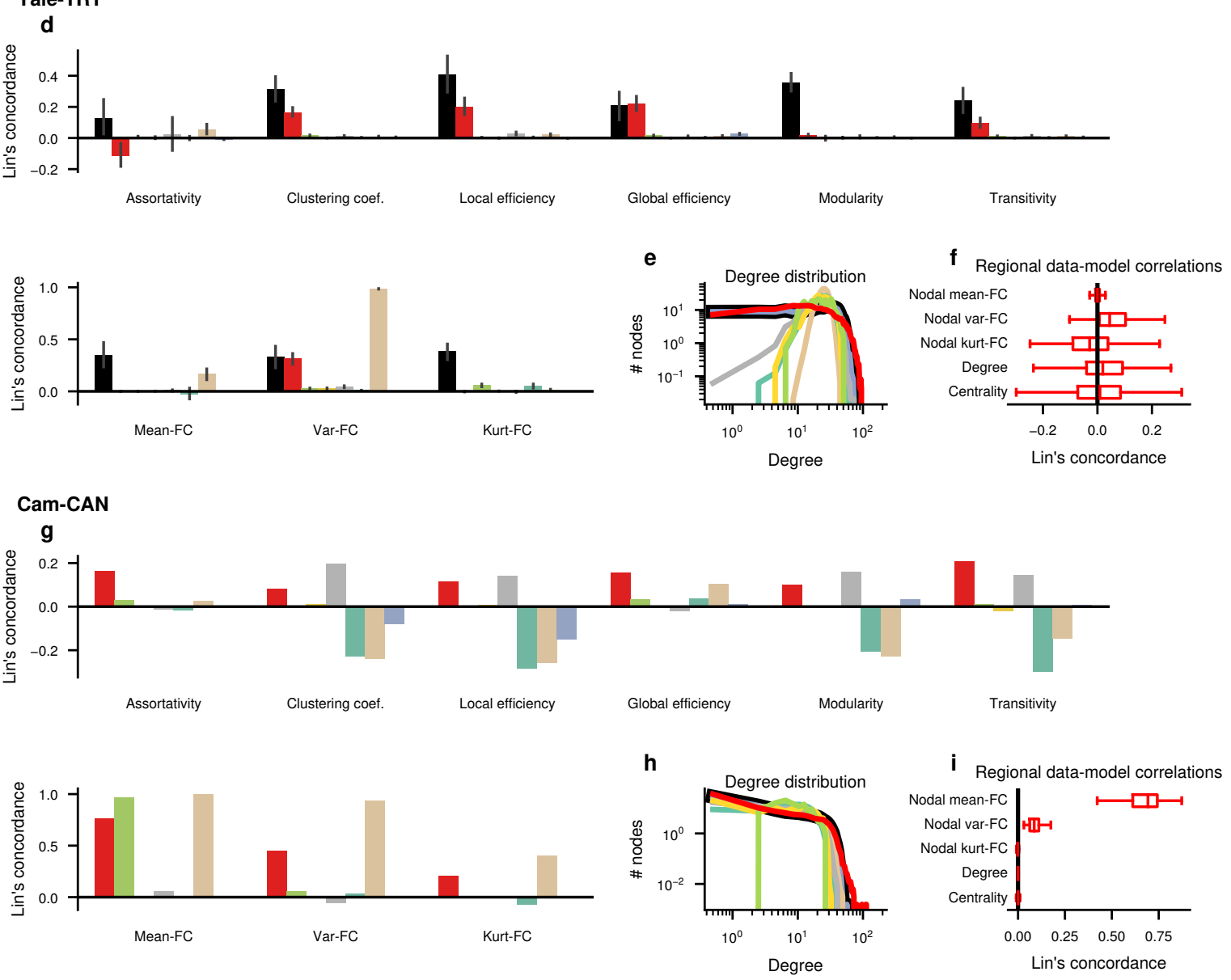

Figure S7 | Comparison of model fits for all datasets. For each dataset, the correlation between model and data is shown for timeseries, weighted, and unweighted graph metrics $(\mathrm{a}, \mathrm{d}, \mathrm{g})$, degree distribution (b,e,h), and distribution of nodal metrics (c,f,i). Statistics of these fits can be found in Table S1. 
Shinn et al.
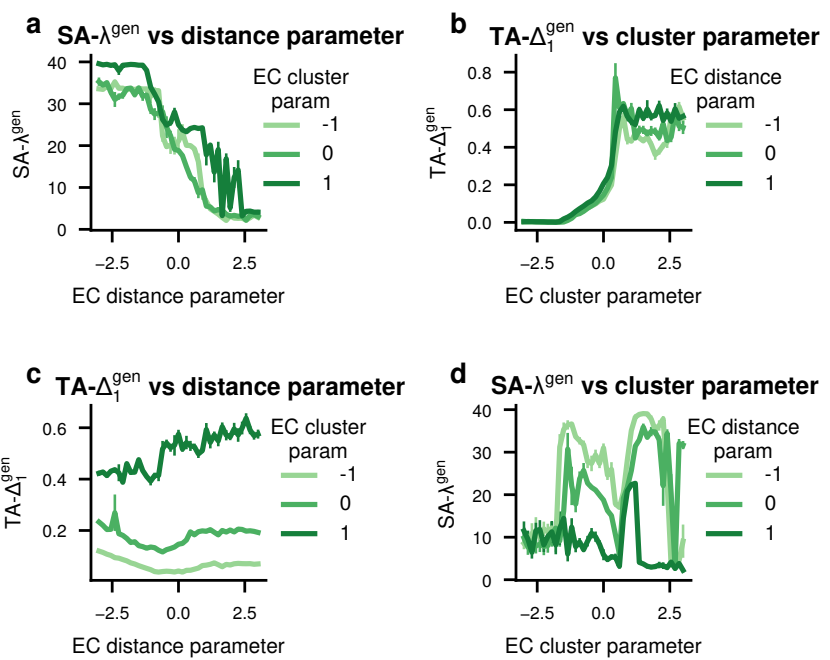

Figure S8 | Relationship between the generative model and economical clustering (EC) model. (a) For three given values of the EC cluster parameter $(\gamma)$, the distance parameter $(\eta)$ was varied and the TA- $\Delta_{1}^{\text {gen }}$ parameter of the best fit generative model is shown. (b) For three given values of the EC distance parameter, the EC cluster parameter was varied and the SA- $\lambda^{\text {gen }}$ parameter of the best fit generative model is shown. Error bars show standard error across 10 simulations of the EC model.

\section{Generative model}

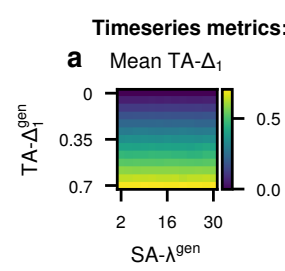

Graph metrics:

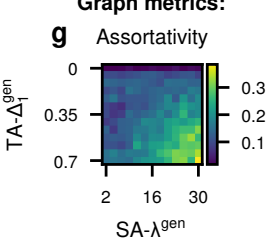

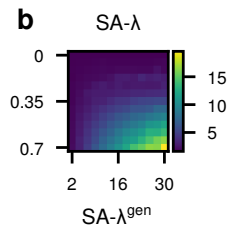

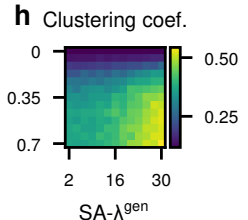

$\mathrm{SA}-\lambda^{\mathrm{gen}}$

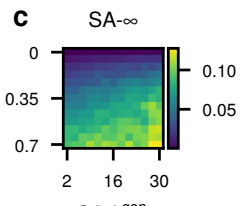

$\mathrm{SA}-\lambda^{\text {gen }}$

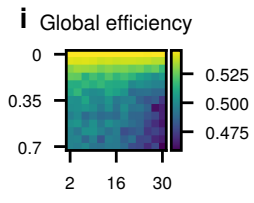

$S A-\lambda^{\text {gen }}$
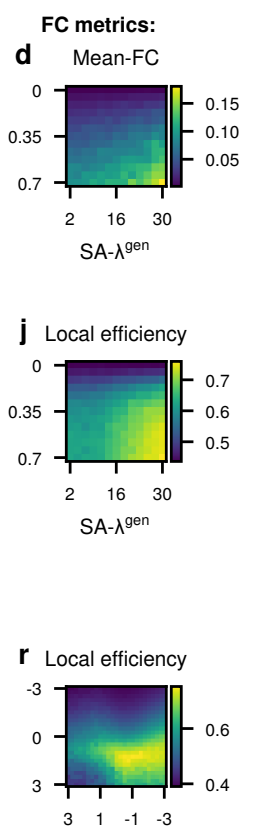

EC distance parameter
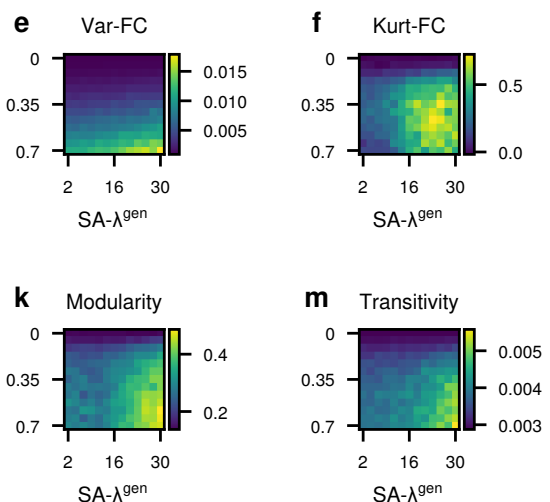

$\mathrm{SA}-\lambda^{\text {gen }}$

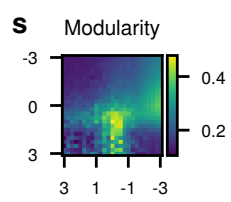

EC distance parameter

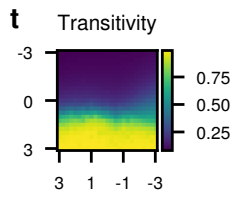

EC distance parameter

Figure S9 | Graph metrics of simulated models. (a-m) The Homogeneous variant of the model was simulated for different values of parameters $S A-\lambda{ }^{g e n}$ and TA- $\Delta_{1}^{g e n}$. For each timeseries metric $(a-c)$, weighted graph metric $(d-f)$, and unweighted graph metric $(g-m)$, the metric value is plotted as a heatmap. (n-t) The economical clustering (EC) model was simulated for different values of its distance and cluster parameters. The value of each of the graph metrics is plotted as a heatmap. 